\title{
O problema de cobertura via geometria algébrica convexa
}

\author{
Leonardo Makoto Mito \\ DISSERTAÇÃO APRESENTADA \\ Instituto DE MATEMÁtica E Estatística \\ UNIVERSIDADE DE SÃO PAULO \\ OBTENÇAO DO TÍTULO \\ MESTRE EM CIÊNCIAS \\ Programa: Matemática Aplicada \\ Orientador: Prof. Dr. Gabriel Haeser
}

Durante o desenvolvimento deste trabalho

o autor recebeu auxílio financeiro do CNPq de março a setembro de 2016 e da FAPESP de outubro de 2016 a fevereiro de 2018, sob o processo no ${ }^{\circ}$ 2016/16999-5.

São Paulo, março de 2018 



\section{O problema de cobertura via geometria algébrica convexa}

Esta versão da dissertação contém as correções e alterações sugeridas pela Comissão Julgadora durante a defesa da versão original do trabalho, realizada em 01/03/2018. Uma cópia da versão original está disponível no Instituto de Matemática e Estatística da Universidade de São Paulo.

Comissão Julgadora:

- Prof. Dr. Gabriel Haeser (orientador) - IME-USP

- Prof. Dr. Marcelo Dias Passos - UFBA

- Prof. Dr. Thadeu Alves Senne - ICT-UNIFESP 



\section{Agradecimentos}

A Deus.

À minha esposa, Rayara, por tudo.

À minha família, em especial meus pais, meu irmão e meus sogros.

Ao Gabriel, pela admirável solicitude ${ }^{1}$, por me orientar de maneira quase montessoriana e pela inspiradora sensatez e genialidade; esses adjetivos são limitantes inferiores.

Aos professores e amigos que conheci no IME, evidenciando o Ernesto, pela disponibilidade e inúmeras sugestões, e o Amorim, por sempre estar diversos níveis acima de mim e me proporcionar excelentes discussões ${ }^{2}$.

Aos professores e amigos do ICT, especialmente ao Angelo, por esculpir um matemático entusiasta de álgebra a partir de algo lamentavelmente disforme e ao Felipe, pelo imenso incentivo (a cursar o mestrado e a estudar otimização, por exemplo) e pelas sugestões; além deles, ao Matheus e à Catarina.

Ao CNPq e à FAPESP, pelo auxílio financeiro, e a todos do departamento de matemática aplicada, pela oportunidade.

\footnotetext{
${ }^{1}$ Ressalto que o tempo de resposta do Gabriel, num meio de comunicação qualquer, é quase zero.

${ }^{2}$ Rima não intencional.
} 



\section{Resumo}

Mito, L. M. O problema de cobertura via geometria algébrica convexa. 2018. Dissertação (Mestrado) - Instituto de Matemática e Estatística, Universidade de São Paulo, São Paulo, 2018.

Este trabalho é focado num problema clássico das Ciências e Engenharia, que consiste em cobrir um objeto por esferas de mesmo raio, a ser minimizado. A abordagem prática usual conta com sérias desvantagens. Logo, faz-se necessário trabalhar com isto de forma diferenciada. A técnica proposta aqui envolve a utilização de resultados célebres da geometria algébrica real, que tem como peça central o positivstellensatz de Stengle e, fazendo a devida relação entre esses resultados e otimização com restrições envolvendo representações naturais por somas de quadrados, é possível reduzir o problema original a um de programação semidefinida não linear. Mas, por contar com particularidades que favorecem a aplicação do paradigma de restauração inexata, esta foi a técnica utilizada para resolvê-lo. A versatilidade da técnica e a possibilidade de generalização direta dos objetos envolvidos destacam-se como grandes vantagens desta abordagem, além da visão algébrica inovadora do problema.

Palavras-chave: problema de cobertura, geometria algébrica real, programação semidefinida, restauração inexata. 



\section{Abstract}

Mito, L. M. The covering problem via convex algebraic geometry. 2018. Thesis (M.Sc.) - Institute of Mathematics and Statistics, University of São Paulo, São Paulo, 2018.

This work is focused on a classic problem from Engineering. Basically, it consists of finding the optimal positioning and radius of a set of equal spheres in order to cover a given object. The common approach to this carries some substantial disadvantages, what makes it necessary to find a different way. Here, we explore some renowned results from real algebraic geometry, which has Stengle's positivstellensatz as one of its central pieces, and SOS optimization. Once the proper link is made, the original problem can be reduced to a nonlinear semidefinite programming one, which has peculiarities that favours the application of an inexact restoration paradigm. We point out the algebraic view and the no use of discretizations as great advantages of this approach, besides the notable versatility and easy generalization in terms of dimension and involved objects.

Keywords: covering problem, real algebraic geometry, semidefinite programming, inexact restoration. 



\section{Lista de Símbolos}

$\mathbb{R}, \mathbb{C}$ e $\mathbb{N}$
$\mathbb{N}^{\star}$
$\|\cdot\|_{2}$
$\mathbb{R}^{n \times m}$
$\mathcal{S}^{n}$
$\mathcal{S}_{+}^{n}$
$\mathcal{S}_{+}^{n}$
$\mathbb{R}[\mathbf{x}]$
$\mathbb{R}_{d}[\mathbf{x}]$
$\mathbb{C}[\mathbf{x}]$
$\Sigma_{n, 2 d}$
$\mathcal{P}_{n, 2 d}$
$\operatorname{diag}\left(a_{1}, \ldots, a_{n}\right)$
$\min (\operatorname{resp} . \max )$
$\operatorname{argmin}$
$\operatorname{tr}(A)$
$\operatorname{det}(A)$
$\lambda_{\text {min }}(A)$
$\sqrt{A}$
$Z(P)$
nonneg $(P)$
$\langle P\rangle$
qmodulo $(P)$
$\operatorname{preorder}(P)$
$\operatorname{monoid}(P)$
$\mathcal{B}(C, r)$
$[\mathbf{x}]_{d}$
$\vec{p}$
$N(d)$
$\mathcal{L}(X, s, y)$
$\mathcal{L}^{\star}(X, s, y)$
$\mathcal{G}(x, y)$
$J_{f}(x)$
$\nabla f(x)$
$P_{S t e}$
$P_{P u t}$

Conjuntos dos números reais, complexos e naturais, resp.

Conjunto dos números naturais sem o zero

Norma Euclidiana em $\mathbb{R}^{n}$

Conjunto das matrizes reais $n \times m$

Conjunto das matrizes reais simétricas $n \times n$

Cone das matrizes reais $n \times n$ simétricas semidefinidas positivas

Cone das matrizes reais $n \times n$ simétricas definidas positivas

Anel de polinômios reais

Anel de polinômios reais de grau até $d$

Anel de polinômios complexos

Cone dos polinômios SOS em $n$ variáveis de grau até $2 d$

Cone dos polinômios não negativos em $n$ variáveis de grau até $2 d$

Matriz diagonal $n \times n$ cujos elementos são $a_{1}, \ldots, a_{n}$

Valor mínimo (resp. máximo) de uma função

Argumento correspondente ao valor mínimo de uma função

Traço de uma matriz $A$

Determinante de uma matriz $A$

Menor autovalor de uma matriz $A$

Raiz quadrada de uma matriz $A \in \mathcal{S}_{+}^{n}$

Conjunto de raízes em comum dos polinômios de $P \subseteq \mathbb{R}[\mathbf{x}]$

Região onde todos os polinômios de $P \subseteq \mathbb{R}[\mathbf{x}]$ são não negativos

Ideal gerado por $P \subseteq \mathbb{R}[\mathbf{x}]$

Módulo quadrático gerado por $P \subseteq \mathbb{R}[\mathbf{x}]$

Pré-ordem gerada por $P \subseteq \mathbb{R}[\mathbf{x}]$

Monóide multiplicativo gerado por $P \subseteq \mathbb{R}[\mathbf{x}]$

Cobertura de esferas com centros em $C$ e raio comum $r$

Vetor de monômios de grau até $d$ sob alguma ordem monomial

Vetor de coeficientes de um polinômio $p$

Binômio $\left(\begin{array}{c}n+d \\ d\end{array}\right)$, fixado $n$ contextualmente

Lagrangiano de um problema de programação semidefinida

Lagrangiano cônico de um problema de programação semidefinida

Função dual ao Lagrangiano

Matriz jacobiana de uma função $f(x)$

Gradiente de uma função $f(x)\left(J_{f}(x)^{t}\right)$

Polinômio referente ao positivstellensatz de Stengle

Polinômio referente ao positivstellensatz de Putinar 



\section{Conteúdo}

1 Preliminares $\quad 13$

1.1 Conjuntos convexos, cones e matrizes . . . . . . . . . . . . . 13

1.1.1 Conjuntos convexos . . . . . . . . . . . . . . . . 13

1.1.2 Cones convexos ....................... 14

1.1.3 Matrizes semidefinidas positivas . . . . . . . . . . . . . 14

1.2 Otimização . . . . . . . . . . . . . . . . . 15

1.2 .1 Programação linear . . . . . . . . . . . . . . . 16

2 O problema de cobertura $\quad 17$

2.1 Caracterização . . . . . . . . . . . . . . . . . . . . . 17

2.2 O tradicional . . . . . . . . . . . . . . . . . . 18

2.3 Uma nova abordagem . . . . . . . . . . . . . . . . . 20

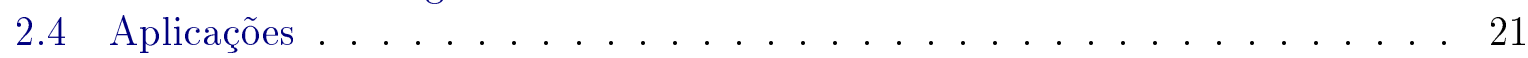

3 Sobre polinômios e somas de quadrados $\quad 23$

3.1 Conjuntos algébricos e Bases de Gröbner . . . . . . . . . . . . . . . . . 24

3.1.1 Uma breve passagem pela teoria das Bases de Gröbner . . . . . . . 25

3.2 Conjuntos semialgébricos e SOS . . . . . . . . . . . 27

3.2 .1 Polinômios não negativos . . . . . . . . . . . . . . 27

3.2 .2 Somas de quadrados . . . . . . . . . . . . . . . 29

3.2 .3 Em uma variável . . . . . . . . . . . . . . . . . . . . . . . . . . . . . . . 31

3.2 .4 Em várias variáveis . . . . . . . . . . . . . . . 34

3.3 Critérios de inviabilidade . . . . . . . . . . . . . . 35

3.3 .1 Estruturação . . . . . . . . . . . . . . . . 36

3.3 .2 O caso linear . . . . . . . . . . . . . . 37

3.3 .3 O caso polinomial . . . . . . . . . . . . . . 38

3.3.4 Conjuntos compactos . . . . . . . . . . . . . 40

4 Programação semidefinida e restauração inexata $\quad 45$

4.1 Programação semidefinida . . . . . . . . . . . . . . . . 45

4.1 .1 Caracterização . . . . . . . . . . . . . . . . 45

4.1.2 Funções Lagrangiana e dual . . . . . . . . . . . . . . . . . 47

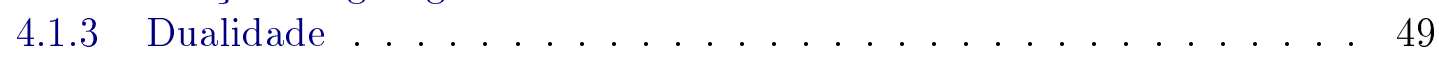

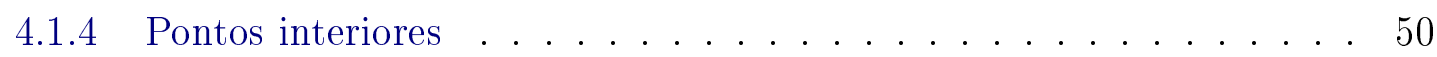

4.1 .5 Solvers computacionais . . . . . . . . . . . . . . . . . . . . 51

4.2 Restauração Inexata . . . . . . . . . . . . . . . . . . . 52

4.2 .1 Uma visão geral . . . . . . . . . . . . . . . . . . . 53

4.2 .2 A fase de restauração . . . . . . . . . . . . . . . . . . . . . 53

4.2 .3 A fase de minimização . . . . . . . . . . . . . 53 
4.2.4 Algoritmo formal . . . . . . . . . . . . . . . . 54

4.2.5 Um comentário sobre convergência . . . . . . . . . . . 55

5 Resultados $\quad 59$

5.1 Acerca do modelo . . . . . . . . . . . . . . . . . 59

5.1 Cobrindo com bolas abertas .................... 61

5.2 Algoritmos . . . . . . . . . . . . . . . . . 66 66

5.2 .1 Menor raio para centros fixos . . . . . . . . . . 66

5.2 .2 Restauração inexata para SDP . . . . . . . . . . . . . . 66

5.2 .3 Sobre a implementação . . . . . . . . . . . . . . . . . 68

5.3 Testes computacionais $($ Putinar + RI $) \ldots \ldots \ldots \ldots$. . . . . . 70

5.3 .1 Um círculo com círculos abertos . . . . . . . . . . . . . 70

5.3.2 Um coração com círculos abertos . . . . . . . . . . . . . . 77

5.4 Infinitos e coberturas abertas . . . . . . . . . . . . 82

5.5 Testes computacionais $($ Stengle $+\mathrm{RI}) \ldots \ldots \ldots \ldots$. . . . . . . . . . . . . . . . 85

5.6 Considerações empíricas, heurísticas e sugestões . . . . . . . . . . 88

5.6 .1 Sobre as variáveis inteiras . . . . . . . . . . . . 89

5.6 .2 Heurísticas . . . . . . . . . . . . . . . . . 89

5.6 .3 Sugestões para trabalhos futuros . . . . . . . . . . . . . 90

6 Conclusão $\quad 91$ 


\section{Introdução}

Este trabalho tem como principal objetivo abordar o clássico problema de cobertura de um objeto utilizando resultados da geometria algébrica real. Existem diversas variantes deste problema, que consiste em encontrar a melhor maneira de cobrir um dado objeto por uma quantidade finita de esferas de mesmo raio ou apenas decidir se um dado conjunto de esferas é ou não uma cobertura. O caso abordado aqui visa encontrar o melhor posicionamento destas, de modo a ter o menor raio possível.

Apesar de nos restringirmos ao problema de cobertura com esferas, uma das principais vantagens do uso desta técnica é que o problema pode ser naturalmente generalizado para objetos de naturezas bastante distintas, desde que suas fronteiras sejam dadas por superfícies de nível de funções polinomiais.

Trata-se de um problema difícil, mesmo para alguns casos particulares aparentemente simples. Como um exemplo de uma das diversas variantes, até 1996 não se sabia qual era o maior círculo que outros oito círculos unitários poderiam cobrir; inclusive, Erich Friedman ${ }^{3}$ cataloga em sua página as melhores coberturas de círculos, triângulos e quadrados, por círculos, já descobertas. Além disso, a formulação do modelo também caracteriza uma tarefa complexa por muitos motivos. Por exemplo, dentre as que envolvem problemas de otimização, está a necessidade de um tratamento para o modelo inicial e a estrutura não diferenciável da formulação, nos casos mais comuns.

A forma com a qual será abordada a questão da cobertura neste projeto é nova e muito interessante por si só ${ }^{4}$, diferindo das maneiras clássicas devido ao ponto de vista geométrico com o qual estará sendo trabalhado e por não necessitar, a priori, de discretizações (que limitam a qualidade da solução). Quanto aos conceitos a serem empregados, faz-se menção ao nullstellensatz de Hilbert, o positivstellensatz de Stengle e Putinar, programação semidefinida e restauração inexata.

Dentre as muitas aplicações para este tema, destaca-se o uso em redes e telecomunicações, podendo também ser utilizada para o posicionamento de satélites e outras áreas das ciências e engenharia. Isto além da própria Matemática, visto que o que será trabalhado vem a ser um tema clássico na literatura, porém abordado de forma nova e diferenciada por esta ser, a princípio, algébrica.

\footnotetext{
${ }^{3}$ Professor associado da Stetson University.

${ }^{4}$ A ideia deste projeto surgiu de uma sugestão dos professores Walter Gómez (UFRO, Chile) e Ernesto Birgin (IME-USP).
} 



\section{Capítulo 1}

\section{Preliminares}

Letras em Blackboard Bold $(\mathbb{R}, \mathbb{C}, \mathbb{K}$ etc.) representam conjuntos numéricos clássicos e corpos, letras caligráficas $(\mathcal{R}, \mathcal{C}, \mathcal{K}$ etc.) são coleções de objetos que não necessariamente possuem alguma estrutura e letras maiúsculas $(R, C, K$ etc.) denotam matrizes e ideais; qualquer exceção será explicitada. Vale mencionar que $\mathbb{N}:=\{0,1,2,3, \ldots\}$ e $\mathbb{N}^{\star}:=\mathbb{N} \backslash\{0\}$, além disso, $\mathbb{R}_{+}$denota o conjunto dos números reais não negativos e $\mathbb{R}_{++}$, dos reais positivos.

$\mathcal{A}^{n}$ é o produto cartesiano de $\mathcal{A}$ por $n-1$ cópias de si mesmo, isto é, seus elementos são da forma $\left(a_{1}, \ldots, a_{n}\right)$, com $a_{i} \in \mathcal{A}$ para todo $i$.

Se $\mathcal{C}$ é um conjunto, $\mathcal{C}^{c}$ denota o seu complementar, $\overline{\mathcal{C}}$ é seu fecho, $\partial \mathcal{C}$ é sua fronteira e $\operatorname{int}(\mathcal{C})$, seu interior; $\emptyset$ é o conjunto vazio.

\subsection{Conjuntos convexos, cones e matrizes}

Um conjunto $A \subseteq \mathbb{R}^{n}$ é um espaço afim se, para quaisquer $x, y \in A$ e $\lambda, \mu \in \mathbb{R}$ com $\lambda+\mu=1, \lambda x+\mu y \in A$; e o conjunto de todas as possíveis combinações afins de um subconjunto $B \subseteq A$,

$$
\operatorname{aff}(B):=\left\{\sum_{i=1}^{m} \lambda_{i} a_{i} \mid m \in \mathbb{N}^{\star}, a_{1}, \ldots, a_{m} \in B, \lambda_{1}, \ldots, \lambda_{m} \in \mathbb{R}, \lambda_{1}+\cdots+\lambda_{m}=1\right\},
$$

é chamado fecho afim de $B$ e é também o menor espaço afim que contém $B$. Linhas, conjuntos unitários, o conjunto vazio, o espaço $\mathbb{R}^{n}$, hiperplanos da forma $H:=\left\{x \in \mathbb{R}^{n} \mid\right.$ $\left.x^{t} a=a_{0}\right\}$ com vetor normal $a \in \mathbb{R}^{n}$ e nível $a_{0} \in \mathbb{R}$, semiespaços na forma $H^{+}:=\{x \in$ $\left.\mathbb{R}^{n} \mid x^{t} a>a_{0}\right\}$ e interseções arbitrárias desses conjuntos são exemplos de subespaços afins de $\mathbb{R}^{n}$.

\subsubsection{Conjuntos convexos}

Um conjunto $S \subseteq \mathbb{R}^{n}$ é convexo se $\lambda x+(1-\lambda) y \in S$ para todo $x, y \in S$ e $\lambda \in[0,1]$. Além disso, dado $T \subseteq \mathbb{R}^{n}$, o conjunto definido por todas as combinações convexas dos elementos de $T$,

$$
\operatorname{conv}(T):=\left\{\sum_{i=1}^{m} \lambda_{i} a_{i} \mid m \in \mathbb{N}^{\star}, a_{1}, \ldots, a_{m} \in B, \lambda_{1}, \ldots, \lambda_{m} \in \mathbb{R}_{+}, \lambda_{1}+\ldots,+\lambda_{m}=1\right\},
$$


é denominado fecho convexo de $T$ e é o menor conjunto convexo que contém $T$. Por exemplo, semiespaços, retas, bolas e interseções de conjuntos convexos são convexos. Todo conjunto convexo fechado $S \neq \emptyset$ possui um hiperplano suporte em cada um dos pontos de sua fronteira, isto é, um hiperplano $H$ que encontra $S$, tal que um de seus semiespaços fechados associados, $\overline{H^{+}}$ou $\overline{H^{-}}$, contém $S$ (esse é chamado de semiespaço suporte). Mais que isso: todo conjunto convexo é a interseção de seus semiespaços suporte. O interior relativo de um conjunto $S$, denotado por $\operatorname{rint}(S):=\left\{x \in \mathbb{R}^{n} \mid \exists \varepsilon>0, B(x, \varepsilon) \cap \operatorname{aff}(S) \subseteq S\right\}$, são os pontos interiores de $S$ quando visto imerso em aff $(S)$ e isto coincide com o interior de $S$ quando aff $(S)=\mathbb{R}^{n}$. Quando os interiores relativos de dois conjuntos convexos não vazios são disjuntos, então eles podem ser separados propriamente por um hiperplano (isto é, pelo menos um dos conjuntos não estará contido no próprio hiperplano); além disso, dois conjuntos convexos não vazios e disjuntos podem ser separados estritamente por um hiperplano (estarão contidos em semiespaços abertos disjuntos), quando um é fechado e outro é compacto.

\subsubsection{Cones convexos}

Cones convexos são subconjuntos $C \subset \mathbb{R}^{n}$ não vazios e fechados quanto a combinações cônicas, isto é, $\alpha v+\beta u \in C$ sempre que $u, v \in C$ e $\alpha, \beta \geqslant 0$; dizemos que $C$ é pontudo se $x,-x \in S$ implica em $x=0$ e é sólido se seu interior é não vazio; além disso, um cone convexo, fechado, pontudo e sólido recebe o nome de cone próprio. O fecho cônico de um conjunto $T \subset \mathbb{R}^{n}$ é a coleção de todas as suas combinações cônicas

$$
\operatorname{cone}(T):=\left\{\sum_{i=1}^{m} \lambda_{i} a_{i} \mid m \in \mathbb{N}^{\star}, a_{1}, \ldots, a_{m} \in B, \lambda_{1}, \ldots, \lambda_{m} \in \mathbb{R}_{+}\right\},
$$

além de ser o menor cone contendo $T$. Cones convexos são, de fato, convexos e definem uma relação de ordem parcial $\preceq$ de modo que $x \preceq y$ se, e somente se, $x-y \in C$; quando $C$ é fechado e $n$-dimensional (ou seja, tem interior não vazio), podemos definir a versão estrita dessa relação $\prec$ como: $x \prec y$ se, e somente se, $x-y \in \operatorname{int}(C)$. Note que é possível separar cones fechados de pontos fora dele estritamente por um hiperplano.

\subsubsection{Matrizes semidefinidas positivas}

Seguindo a notação usual, $\mathbb{R}^{n \times m}$ remete ao espaço vetorial das matrizes com $n$ linhas, $m$ colunas, entradas em $\mathbb{R}$ e as operações usuais; se $X$ e $Y$ são elementos desse conjunto, então $X^{t}$ é a transposta de $X$ e o produto interno usual de $X$ por $Y$ é definido como $X \cdot Y:=\operatorname{tr}\left(X^{t} Y\right)$, isto é, o traço de $X^{t} Y$; note que, se $m=1$, então $X \cdot Y=X^{t} Y$.

Um fato digno de menção é que toda matriz simétrica $A$ possui uma decomposição da forma

$$
A=\sum_{i=1}^{n} \lambda_{i} u_{i} u_{i}^{t}=P D P^{t}
$$

onde $\lambda_{i}$ são os autovalores de $A$ com autovetores associados $u_{i}$ que formam uma base ortonormal de $\mathbb{R}^{n}, P$ é a matriz formada com os $u_{i}$ nas colunas e $D$ é uma matriz com os $\lambda_{i}$ na diagonal principal; essa representação é conhecida como decomposiçãa espectral de A.

Denotamos por $\mathcal{S}^{n}$ o subespaço vetorial das matrizes simétricas reais $n \times n$ e $\mathcal{S}_{+}^{n}$, o subespaço vetorial de $\mathcal{S}^{n}$ contendo as matrizes semidefinidas positivas, isto é, $A \in \mathcal{S}_{+}^{n}$ 
quando sua forma quadrática associada $z^{t} A z$ é não negativa para todo $z \in \mathbb{R}^{n}$. Como $\mathcal{S}_{+}^{n}$ é um cone convexo, esse conjunto induz uma relação de ordem parcial $\succeq$ (de Löwner). Inclusive, deste ponto em diante, a expressão " $A \succeq 0$ " envolvendo uma matriz $A$ significará que $A \in \mathcal{S}_{+}^{n}$. Quando $z^{t} A z>0$ para todo $z \in \mathbb{R}^{n}, A$ é dita ser definida positiva e isto será denotado, é claro, por " $A \succ 0$ "; o conjunto das matrizes reais definidas positivas será representado por $\mathcal{S}_{++}^{n}$, que inclusive é o interior de $\mathcal{S}_{+}^{n}$.

Teorema 1. Seja $A=\left[a_{i j}\right]_{i, j \in\{1, \ldots, n\}}$ uma matriz simétrica real $n \times n$. São equivalentes as seguintes afirmações:

1. A é semidefinida positiva;

2. Se $\lambda$ é autovalor de A, então $\lambda \geqslant 0$;

3. Para todo subconjunto $J$ de $\{1, \ldots, n\}$, a matriz $\left[a_{i j}\right]_{i, j \in J}$ tem determinante não negativo [Pru86];

4. Existe $G \in \mathbb{R}^{n \times n}$ tal que $A=G G^{t}$ (decomposição de Gram), além disso, $G$ pode ser escolhida em $\mathbb{R}^{n \times r}$, onde $r=\operatorname{rank}(A)$;

5. $N A N^{t}$ é semidefinida positiva para toda matriz $N$ não singular.

Note que, pelo item 2 do Teorema 1 , se $A \succeq 0$, então existe uma decomposição espectral $A=P D P^{t} \operatorname{com} D=\operatorname{diag}\left(\lambda_{1}, \ldots, \lambda_{n}\right)$ possuindo apenas entradas não negativas. Logo, podemos definir a matriz $\sqrt{D}:=\operatorname{diag}\left(\sqrt{\lambda_{1}}, \ldots, \sqrt{\lambda_{n}}\right)$ e, consequentemente, a raiz quadrada de $A$ como $\sqrt{A}:=P \sqrt{D} P^{t}$, que satisfaz a relação $A=\sqrt{A} \sqrt{A}$, pois $P$ é ortonormal.

A seguir, exibiremos algumas propriedades do produto interno fixado em $\mathbb{R}^{n \times n}$.

Proposição 2. Se $X, Y \in \mathbb{R}^{n \times n}$, então:

1. $X \cdot Y=\operatorname{tr}\left(X^{t} Y\right)=\sum_{i=1}^{n} \sum_{j=1}^{n} X_{i j} Y_{i j}$;

2. Se $z \in \mathbb{R}^{n}$, então $\left(z z^{t}\right) \cdot X=z^{t} X z$;

3. $X \succeq 0$ se, e somente se, $X \cdot Y \geqslant 0$, para todo $Y \succeq 0$;

4. $X \succ 0$ se, e somente se, $X \cdot Y>0$, para todo $Y \succeq 0, Y \neq 0$;

5. Sejam $X, Y \in \mathcal{S}_{+}^{n}$. Então $A \cdot B=0$ se, e somente se, $A B=0$.

\subsection{Otimização}

Um problema de otimização geral é composto por uma função objetivo $f: \mathbb{R}^{n} \rightarrow$ $\mathbb{R}$, uma região factivel $\mathcal{K}$ definida por um conjunto de restrições e, quando existir, um conjunto de soluções, isto é, um subconjunto de $\mathcal{K}$ cujos elementos adquirem, pelo menos em alguma vizinhança, os menores valores quando avaliados por $f$. A notação usual para este tipo de problema é:

$$
\min f(x)
$$

sujeito a $x \in \mathcal{K}$. 


\subsubsection{Programação linear}

A forma primal de um problema padrão de programação linear (LP) é normalmente escrita como

$$
\begin{array}{r}
\min c^{t} x \\
\text { sujeito a } A x=b \\
x_{i} \geqslant 0, \forall i \in\{1, \ldots, n\},
\end{array}
$$

onde $m$ e $n$ são naturais tais que $m \leqslant n, c \in \mathbb{R}^{n}$ é o chamado custo do problema, $b \in \mathbb{R}^{m} \mathrm{e}$ $A \in \mathbb{R}^{m \times n}$ são dados que definem as restrições e $x \in \mathbb{R}^{n}$ é a variável de decisão. A última restrição do problema será abreviada para $x \geqslant 0$ quando não houver ambiguidade.

A região factível $\mathcal{K}$ do Problema (1.1) é uma interseção de hiperplanos (subespaços afins) e o ortante não negativo em $\mathbb{R}^{n}$ (um cone), ou seja, é um conjunto necessariamente convexo chamado de poliedro. Um conceito extremamente útil sobre LPs é o fato de sempre existir um outro problema também linear associado, denominado dual:

$$
\begin{array}{r}
\max b^{t} y \\
\text { sujeito a } A^{t} y \leqslant c
\end{array}
$$

onde $y \in \mathbb{R}^{m}$ é a variável de decisão. São amplamente conhecidas as propriedades que relacionam (1.1) e (1.2). Por exemplo, o fato dos valores dos pontos viáveis (pela função objetivo) do problema primal serem limitantes superiores para as soluções do dual, que por sua vez tem pontos viáveis cujos valores limitam inferiormente as soluções do primal (dualidade fraca) e, caso um deles seja limitado, então ambos o são e têm soluções iguais (dualidade forte). 


\section{Capítulo 2}

\section{O problema de cobertura}

O objetivo do principal motivador deste trabalho é fácil de enunciar: cobrir um objeto com esferas da melhor forma possível. Mas como o significado desta frase está sujeito ao contexto em que o problema for apresentado, este capítulo é dedicado a determinar e formular matematicamente o caso particular de nosso interesse. Porém, mesmo um caso fixo permite diversas abordagens diferentes, boa parte delas envolvendo discretizações e cobertura de pontos; iniciaremos apresentando uma delas, suas principais vantangens e desvantagens e, em seguida, introduziremos uma nova abordagem algébrica para o problema em questão.

\subsection{Caracterização}

Nosso ambiente de trabalho será o espaço real em $n$ dimensões munido da Norma Euclidiana, $\|\cdot\|_{2}$. Dado um objeto, a princípio qualquer, denotado por $V$ e $m \in \mathbb{N}^{\star}$, desejamos encontrar os melhores centros $C:=\left\{c_{1}, \ldots, c_{m}\right\} \subset \mathbb{R}^{n}$ e o menor raio $r \geqslant 0$ de modo que a coleção de esferas

$$
B\left(c_{i}, r\right):=\left\{x \in \mathbb{R}^{n} \mid\left\|x-c_{i}\right\|_{2} \leqslant r\right\}, i \in\{1, \ldots, m\}
$$

forme uma cobertura para $V$, ou seja, denotando por $\mathcal{B}(C, r)$ o conjunto resultante da união de todos os $B_{i}$, buscamos uma solução do problema de otimização

$$
\begin{array}{r}
\min f\left(r, c_{1}, \ldots, c_{m}\right) \\
\text { sujeito a } V \subseteq \mathcal{B}(C, r) \\
r \geqslant 0,
\end{array}
$$

onde a função objetivo $f: \mathbb{R}^{n m+1} \rightarrow \mathbb{R}$ é o que dá sentido é expressão "melhor forma", podendo ser, por exemplo, uma representação da área de $\mathcal{B}(C, r)$ ou apenas o raio, caso o interesse seja a menor área total ou a menor área individual, respectivamente; note que estes dois objetivos são diferentes e que o posicionamento dos centros está implicitamente relacionado com o raio mínimo.

Exemplo 1. A figura de contorno azul e preenchimento vermelho abaixo é $V$ e os três círculos alaranjados formam a cobertura com círculos de menor raio possivel $\left(r^{\star}=\sqrt{0.75}\right)$ para $V$, porém claramente não é a de menor área, pois se todos os centros estivessem em $\overrightarrow{0}$ e o raio comum fosse 1 , teríamos a cobertura de menor área possível; por outro lado, a de menor área obviamente não tem o menor raio.

Portanto, minimizar a área e o raio são duas tarefas completamente distintas. 


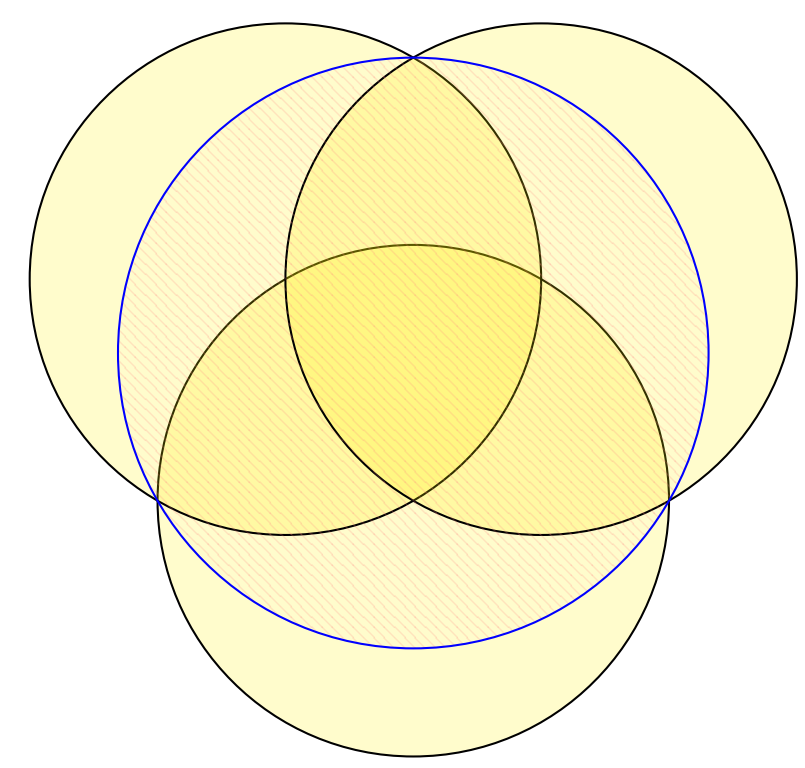

Figura 2.1: Exemplo de cobertura com o menor raio possível, mas não a menor área.

\section{$2.2 \quad \mathrm{O}$ tradicional}

Uma das maneiras clássicas na Engenharia de se trabalhar com o modelo (2.1), baseado em [VLX15], é transformá-lo num problema min-max-min da seguinte forma: defina a função

$$
d(v, C):=\min _{i=1, \ldots, m}\left\|v-c_{i}\right\|_{2}
$$

que representa a distância mínima entre um ponto $v \in V$ e o conjunto discreto $C$ (se $d(v, C) \leqslant r$, o ponto $v$ está coberto por pelo menos uma das esferas). Sendo assim, a função

$$
D_{V}(C):=\max _{v \in V} d(v, C)
$$

representa a maior distância entre $V$ e $C$ (então se $D_{V}(C)>r$ a superfície não está inteiramente coberta). O problema min-max-min consiste, portanto, em minimizar a função $D_{V}(C)$ em $C$, isto é, encontrar o conjunto de centros $C$ cuja região formada $\mathcal{B}\left(C, D_{V}(C)\right)$ está mais próxima de cobrir $V$. Formalmente:

$$
\min _{C} \max _{v \in V} \min _{i=1, \ldots, m}\left\|v-c_{i}\right\|_{2}
$$

Para resolver o Problema (2.2) numericamente, utiliza-se o artifício de aproximar $V$ por um conjunto finito de pontos $V^{\star}:=\left\{v_{1}, \ldots, v_{k}\right\}$ e, para cada um destes, defina $z_{j}(C):=d\left(v_{j}, C\right), j=1, \ldots, k \mathrm{e}$

$$
z(C)=\max _{j=1, \ldots, k} z_{j}(C)=D_{V^{\star}}(C) .
$$

Desta forma, podemos explicitar condições para que as funções $z_{j}(C)$ e $z(C)$ estejam bem definidas e tratá-las como restrições, obtendo o seguinte problema equivalente, com $\phi(y):=\max \{0, y\}:$

$\min z$

$$
\text { sujeito a } \sum_{i=1}^{m} \phi\left(z-\left\|v_{j}-c_{i}\right\|_{2}\right)>0, j \in\{1, \ldots, k\} \text {. }
$$


A partir deste ponto, existe uma coleção de métodos capazes de computar a solução. O foco é a abordagem. Note que foi necessário discretizar o objeto para tornar o método computável, o que, se não for feito de maneira satisfatória, pode levar a uma solução inconsistente. Um exemplo simples para visualizar isso é tomar $V$ como um círculo discretizado com pontos interiores. Neste caso, uma cobertura da discretização pode ser inviável para o problema original, ou seja, pode não cobrir o círculo todo, apesar de ser solução para o modelo, como no caso abaixo:

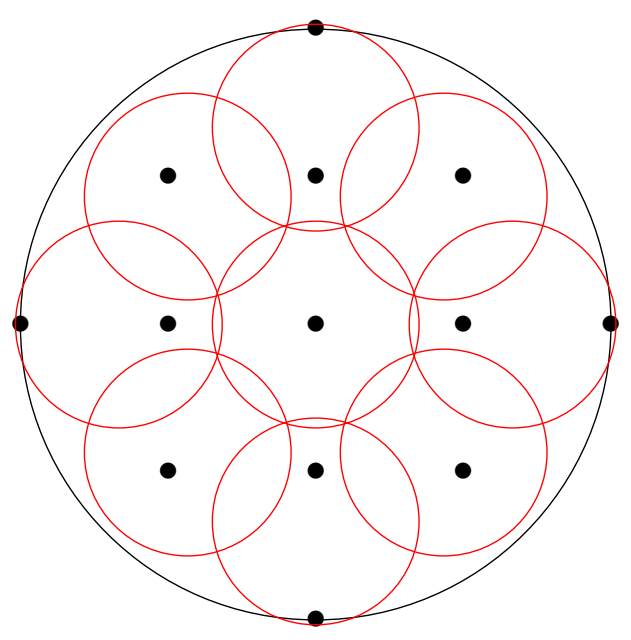

Figura 2.2: Um exemplo da desvantagem na discretização interior.

Um modo de contornar isto seria incluir alguns pontos exteriores próximos à discretização. Porém, mesmo garantindo que a cobertura calculada pelo método envolva o círculo todo, fazer isso permite que a solução do modelo seja não ótima para o problema original. Isto é ilustrado pela figura abaixo, onde a cobertura azul, que é evidentemente melhor que a vermelha, apesar de cobrir o objeto, não cobre a discretização.

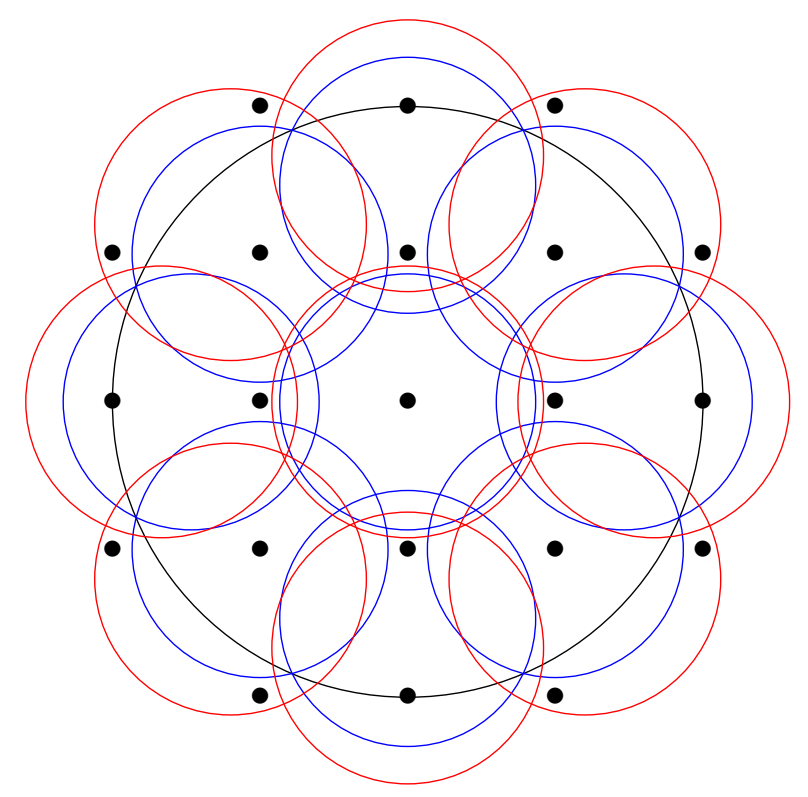

Figura 2.3: Um exemplo da desvantagem na discretização exterior. 
A forma mais prática de se evitar essas duas falhas é utilizar um número suficientemente grande de pontos na discretização, o que implica no grande aumento do tempo computacional necessário para se obter a resposta; além disso, muitas vezes este tipo de abordagem não tira proveito das particularidades de $V$, mas dependendo da aplicação, isto pode ser uma vantagem, por também não ser prejudicada por tais peculiaridades. Em certos casos, os motivos citados tornam a discretização indesejada e trazem à tona a necessidade de uma abordagem que trate o problema como ele é: uma cobertura de conjuntos infinitos com outros conjuntos infinitos.

\subsection{Uma nova abordagem}

O modelo que introduziremos nesta seção foi sugerido por Ernesto Birgin e Walter Gómez, assim como a ferramenta algébrica para lidar com ele. A linguagem desta abordagem é composta por polinômios e uma das suposições necessárias para que tudo funcione bem, ao preço de resultados mais fracos, é que $V$ seja um conjunto semialgébrico básico fechado, isto é, que existam polinômios reais $q_{i}(\mathbf{x}), i \in\{1, \ldots, t\}$, tais que $V=\left\{x \in \mathbb{R}^{n} \mid q_{i}(x) \geqslant\right.$ $0, \forall i \in\{1, \ldots, t\}\}$. Agora, sejam $p_{i}(x):=\left\|x-c_{i}\right\|_{2}^{2}-r, i \in\{1, \ldots, m\}$, os polinômios que definem as esferas em $\mathcal{B}(C, r)$ (aqui trocamos $r^{2}$ por $r$ mais a restrição $r \geqslant 0$ com o propósito de "linearizar" o problema). Então

$V \subseteq \mathcal{B}(C, r) \Leftrightarrow \mathcal{K}:=\left\{x \in \mathbb{R}^{n} \mid q_{i}(x) \geqslant 0, p_{j}(x)>0, \forall i \in\{1, \ldots, t\}, \forall j \in\{1, \ldots, m\}\right\}=\emptyset$,

isto é, $V$ está contido em $\mathcal{B}(C, r)$ quando nenhum de seus pontos está fora de $\mathcal{B}(C, r)$. Aqui, fazemos a livre correspondência entre polinômios e suas funções polinomiais associadas.

Tomando $f\left(r, c_{1}, \ldots, c_{m}\right)=r$, podemos reescrever (2.1) como um problema de otimização da seguinte forma:

$$
\begin{array}{r}
\min r \\
\text { sujeito a } \mathcal{K}=\emptyset \\
r \geqslant 0,
\end{array}
$$

onde as variáveis são $r, c_{1}, \ldots, c_{m}$ e, inclusive, $\mathcal{K}=\mathcal{K}(r, C)$. Existem ferramentas que podem auxiliar na resolução de (2.4). Dentre elas, estão alguns célebres resultados da geometria algébrica real, que serão introduzidos no capítulo seguinte.

Algo importante a se notar é que o modelo (2.4) é muito versátil, em diversos sentidos, o que nos move a destacar algumas modificações possíveis:

- A dimensão do problema torna-se apenas um problema computacional, pois o modelo (e toda a teoria) é invariante em relação a $n$.

- Esta representação do conjunto $V$ traz boas propriedades computacionais (veja a Seção 3.3.4), mas a priori é possível introduzir restrições com os símbolos $<,=\mathrm{e} \neq$ sem muitas dificuldades teóricas adicionais.

- É possível trocar as esferas por quaisquer outras regiões limitadas (abertas ou fechadas) que podem ser descritas por curvas de nível de apenas um polinômio (por exemplo, outras normas). 


\subsection{Aplicações}

Um exemplo de problema real que se enquadra no modelo (2.1) é o da alocação ótima de torres de transmissão, comum em problemas de telefonia móvel, em que o raio das esferas é o alcance do transmissor e a região a ser coberta é o contorno de uma cidade pré-determinada. Assumindo que o custo de uma torre é muito maior que o custo de uma antena, faz sentido o seu número ser fixo; neste caso, comprar antenas potentes demais pode implicar em uma grande perda de capital. Logo, decidir onde alocar as torres e a pior antena possível se enquadra no nosso modelo. O contorno do mapa através de um polinômio pode ser obtido com interpolação polinomial. Um exemplo análogo em três dimensões é a alocação de roteadores Wi-Fi num edifício, que pode ser descrito com apenas três polinômios quadráticos. 



\section{Capítulo 3}

\section{Sobre polinômios e somas de quadrados}

O propósito deste capítulo, baseado majoritariamente em [Lau09] e [PBT13], é apresentar alguns resultados clássicos da geometria algébrica real, que fundamentarão a abordagem escolhida no primeiro capítulo. Aqui apresentaremos alguns resultados clássicos da álgebra comutativa e geometria algébrica, definiremos o que são conjuntos algébricos e semialgébricos, polinômios não negativos e sua relação com somas de quadrados, inclusive como computar esses termos. Além disso, exibiremos algumas maneiras de caracterizar teoricamente conjuntos algébricos e semialgébricos vazios e suas aplicações no problema (2.4).

Seguindo a notação clássica, $\mathbb{R}\left[x_{1}, \ldots, x_{n}\right]$ denota o anel de polinômios em $n$ variáveis sobre o corpo real, com a adição e o produto usuais e, quando não houver necessidade de explicitar as variáveis, esta notação será abreviada para $\mathbb{R}[\mathbf{x}]$, com $\mathbf{x}=\left(x_{1}, \ldots, x_{n}\right)$.

Um elemento $p$ de $\mathbb{R}[\mathbf{x}]$ pode ser escrito como $p:=\sum_{i \in \mathbb{N}} c_{i} \mathbf{x}^{\alpha_{i}}, \operatorname{com} \mathbf{x}^{\alpha_{i}}:=x_{1}^{\alpha_{i 1}} \ldots x_{n}^{\alpha_{i n}}$, onde os coeficientes $c_{i} \in \mathbb{R}$ são, em quantidade finita, não nulos e $\alpha_{i} \in \mathbb{N}^{n}$; cada parcela desta soma, com $c_{i} \neq 0$, é um termo, que é composto também por um monômio $\mathbf{x}^{\alpha_{i}}$. Definimos $\mathcal{M}(p)$ como o conjunto dos monômios de $p, \operatorname{deg}\left(\mathbf{x}^{\alpha_{i}}\right):=\sum_{j=1}^{n} \alpha_{i j}$ como o grau de $\mathbf{x}^{\alpha_{i}}$ e, analogamente, $\operatorname{deg}(p):=\max \{\operatorname{deg}(m) \mid m \in \mathcal{M}(p)\}$ denota o grau de $p$; quando todos os termos de um polinômio têm o mesmo grau, ele é chamado de homogêneo ou forma. Além disso, será feita a livre correspondência entre os elementos deste anel e suas funções polinomiais homônimas associadas $p: \mathbb{R}^{n} \rightarrow \mathbb{R}$, conforme for conveniente.

Definição 1 (Ordem monomial). Se $\mathcal{M}$ denota um conjunto de monômios, então uma boa ordem $\preceq$ (total, que satisfaz o princípio da boa ordenação) em $\mathcal{M}$ é chamada de ordem monomial sobre $\mathcal{M}$ quando, dados quaisquer $a, b \in \mathcal{M}$ tais que $a \preceq b$ (a é igual ou precede b), então para todo $c \in \mathcal{M}$ vale que ac $\preceq b c$.

O monômio líder de $p$ relativo a $\preceq$ é $\operatorname{ml}(p):=\max _{\preceq} \mathcal{M}(p)$, seu coeficiente líder associado é denotado por $\mathrm{cl}(p)$ e, por último, $\mathrm{tl}(p):=\operatorname{ml}(p) \mathrm{cl}(p)$ é o termo líder de $p$. Um bom exemplo de ordem monomial é a seguinte:

Definição 2 (Ordem lexicográfica). Dados $a, b \in \mathcal{M}$ tais que $a=x^{\alpha}$ e $b=x^{\beta}$, a ordem monomial lexicográfica $\preceq_{L}$ impõe que $a \preceq_{L} b$ quando, definindo $\gamma:=\beta-\alpha$, existe $k \in \mathbb{N}$ tal que $\gamma_{i}=0$, se $i<k$ e $\gamma_{k}<0$. Uma variação desta é a ordem $\preceq_{G L}$ chamada lexicográfica graduada, segundo a qual os monômios são ordenados primeiramente pelo grau e, caso haja igualdade, vale a lexicográfica.

Segundo a ordem lexicográfica graduada, 1 precede qualquer outro monômio e $\mathbf{x}^{(1,1)}=$ $x_{1} x_{2} \preceq x_{2}^{2}=\mathbf{x}^{(0,2)}$, pois $\gamma=(0,2)-(1,1)=(-1,1)$ e $k=1$ na notação da definição. 
Daqui em diante, fixaremos a ordem lexicográfica graduada em anéis de polinômios, por ser a mais intuitiva dentre todas as outras.

Note que, munindo $\mathcal{M}$ de uma ordem monomial, é possível caracterizar um polinômio $p$ apenas por seu vetor de coeficientes $\vec{p}:=\left(c_{i}\right)_{i \in \mathbb{N}}$, o que estabelece uma relação injetiva entre $\mathbb{R}[\mathbf{x}]$ e $\mathbb{R}^{\infty}$; além disso, as definições acima podem ser estendidas para qualquer corpo, além do real (por exemplo, $\mathbb{C}[\mathbf{x}]$ é o anel de polinômios sobre o corpo complexo).

\subsection{Conjuntos algébricos e Bases de Gröbner}

Conjuntos algébricos são aqueles definidos por zeros de polinômios, como hiperplanos, círculos, pontos ou quaisquer outras curvas de nível de funções polinomiais. O propósito desta seção é expor alguns resultados referentes à vacuidade de um conjunto algébrico definido por polinômios complexos, algo que é amplamente conhecido, e um pouco da teoria das Bases de Gröbner.

Definição 3 (Conjunto algébrico). Dado $P \subseteq \mathbb{C}[\boldsymbol{x}]$, o zero-lócus de $P$ é o conjunto $Z(P):=\left\{x \in \mathbb{C}^{n} \mid p(x)=0, \forall p \in P\right\}$ e um conjunto $A \subseteq \mathbb{C}^{n}$ é dito algébrico se $A=Z(S)$, para algum $S \subseteq \mathbb{C}[\boldsymbol{x}]$; quando um conjunto algébrico não pode ser escrito como união de dois ou mais subconjuntos algébricos próprios (irreducibilidade), ele é chamado de variedade algébrica.

Se $A=Z(f)$ e $B=Z(g)$ são conjuntos algébricos, então $A \cap B=Z(f, g)$ e $A \cup B=$ $Z(f g)$. Indutivamente, concluímos que uniões e interseções finitas de conjuntos algébricos são também conjuntos algébricos.

Definição 4 (Ideal). Um subconjunto I de $\mathbb{C}[\boldsymbol{x}]$ é um ideal quando:

1. $0 \in I$;

2. $a, b \in I$ implica que $a+b \in I$;

3. $a \in I$ e $b \in \mathbb{C}[\boldsymbol{x}]$ implica em $a b \in I$.

Somas e interseções de ideais são ideais e, dado um subconjunto $P$ de $\mathbb{C}[\mathbf{x}]$, o conjunto

$$
\langle P\rangle:=\left\{\sum_{p \in Q} p h_{p} \mid Q \text { é um subonjunto finito de } P \text { e } h_{p} \in \mathbb{C}[\mathbf{x}], \forall p \in Q\right\}
$$

é um ideal e é dito o ideal gerado por $P$. Os zeros de $P$ são exatamente os zeros de $\langle P\rangle$, isto é, equações da forma $q=0$ com $q$ no ideal gerado por $P$ podem ser vistas como redundantes ao sistema polinomial $\{p(x)=0, \forall p \in P\}$ (quando não houver ambiguidade, este símbolo será abreviado pela expressão " $P=0$ ").

David Hilbert, em 1893, propôs e provou um dos resultados mais importantes da álgebra comutativa, conhecido como nullstellensatz, mas o que nos interessa aqui é sua forma mais fraca, que constitui um critério de inviabilidade para um sistema de polinômios complexos e, consequentemente, de vacuidade para um conjunto algébrico complexo.

Teorema 3 (Weak nullstellensatz). Seja $P:=\left\{p_{1}, \ldots, p_{m}\right\} \subset \mathbb{C}[\boldsymbol{x}]$, ent $\tilde{a} o Z(P)=\emptyset$ se, e somente se, $1 \in\langle P\rangle$.

Demonstração. Uma demonstração elementar pode ser encontrada em [Man04]. 
Um sistema polinomial real $P_{\mathbb{R}}=0$ (com coeficientes reais e buscando apenas raízes reais) é um caso particular do complexo e, portanto, a presença da unidade no ideal $\left\langle P_{\mathbb{R}}\right\rangle$ é uma condição suficiente de inviabilidade, porém não necessária. Por exemplo, o conjunto $\left\{x \in \mathbb{R} \mid x^{2}+1=0\right\}$ é vazio, mas $p=x^{2}+1$ não é $\mathbb{R}[x]$-múltiplo de 1 ; contudo, $-1=(-1) \cdot p+x^{2}$, que é a soma de um elemento de $\langle p\rangle$ com um quadrado. Maiores detalhes sobre isso serão dados na terceira seção deste capítulo (Corolário 19).

\subsubsection{Uma breve passagem pela teoria das Bases de Gröbner}

Antes de continuarmos, é válido mencionar pelo menos um método computacional capaz de verificar o critério do Teorema 3. Para isso, relembramos o algoritmo da divisão no anel de polinômios.

Teorema 4 (Algoritmo da Divisão). Dado $F:=\left\{f_{1}, \ldots, f_{k}\right\} \subset \mathbb{C}[\boldsymbol{x}]$, para cada $f \in \mathbb{C}[\boldsymbol{x}]$, existem $a_{1}, a_{2}, \ldots, a_{k}$ e $r \in \mathbb{C}[\boldsymbol{x}]$, tais que $f=a_{1} f_{1}+\ldots+a_{k} f_{k}+r$ e $\mathrm{ml}(r)$ não divide nenhum $\mathrm{ml}\left(f_{i}\right)$. O polinômio $r$ é o resto da divisão de $f$ por $F$ e será denotado por $r_{F}(f)$.

A alternativa mais intuitiva para decidir a inviabilidade de $P=0$ é simplesmente computar $r_{P}(1)$ e verificar se é ou não nulo, mas esse resto nem sempre é único com respeito à permutação. Então, $r_{P}(1) \neq 0$ não implica em $1 \notin\langle P\rangle$, pois a primeira afirmação está sujeita à ordem em que essa divisão é feita.

Exemplo 2. Tome $f=x y^{2}-x, g_{1}=x y-y$ e $g_{2}=y^{2}-x \in \mathbb{K}[x, y]$ e fixe a ordem lexicográfica com $y \prec x$. Ao dividir $f$ por $g_{1} e$, em seguida, por $g_{2}$, utilizando o algoritmo da divisão, obtêm-se que $f=y g_{1}+g_{2}$, onde o resto é nulo, mas ao dividir primeiro por $g_{2}$ e depois por $g_{1}$, pode-se obter $f=x g_{2}+0 . g_{1}+x^{2}-x$, ou seja, deixa como resto o polinômio $x^{2}-x \neq 0$.

Felizmente, Bruno Buchberger e Wolfgang Gröbner, em 1965, concluíram os fundamentos da teoria das Bases de Gröbner, que nos permite avançar com o raciocínio anterior.

Definição 5 (Base de Gröbner). Seja I um ideal de $\mathbb{C}[\boldsymbol{x}]$. Diz-se que um subconjunto finito $G$ de I é uma Base de Gröbner quando, dado qualquer $f \in \mathbb{C}[\boldsymbol{x}], r_{G}(f)=0$ se, e somente se, $f \in I$.

Ressaltamos que toda Base de Gröbner de um ideal $I$ é, de fato, um gerador de $I$ e um resultado ainda mais importante é que todo ideal não nulo de $\mathbb{C}[\mathbf{x}]$ possui uma Base de Gröbner (veja [Cou09] para mais detalhes), o que resulta no famoso Teorema das Bases de Hilbert como corolário, que afirma que todo ideal admite um número finito de geradores. A principal contribuição de Buchberger foi o seguinte resultado, que é um critério de decisão computacionalmente viável para estabelecer um critério de parada para o algoritmo apresentado em seguida.

Teorema 5 (Critério de Buchberger). Dado um ideal $I \subseteq \mathbb{C}[\boldsymbol{x}]$, um conjunto $G=$ $\left\{g_{1}, \ldots, g_{s}\right\} \subseteq I$ é uma Base de Gröbner para $I$ se, e somente se, $r_{G}\left(S\left(g_{i}, g_{j}\right)\right)=0$, para quaisquer $g_{i}, g_{j} \in G$.

O polinômio dado por

$$
S\left(g_{i}, g_{j}\right):=\operatorname{mmc}\left(\mathrm{ml}\left(g_{i}\right), \mathrm{ml}\left(g_{j}\right)\right) \frac{g_{i}}{\mathrm{tl}\left(g_{i}\right)}-\operatorname{mmc}\left(\mathrm{ml}\left(g_{i}\right), \mathrm{ml}\left(g_{j}\right)\right) \frac{g_{j}}{\mathrm{tl}\left(g_{j}\right)}
$$

é chamado S-polinômio de $g_{i}$ e $g_{j}$. 
Para finalizar, faremos menção ao célebre Algoritmo de Buchberger, que computa esses geradores, dado qualquer ideal; isso complementa o algoritmo da divisão para determinar computacionalmente se o conjunto algébrico definido por $P$ é vazio ou não. Ele é intuitivamente induzido do Critério de Buchberger e visa incluir os restos da divisão dos S-polinômios de cada iteração por $G$, nele mesmo, culminando em uma Base de Gröbner:

Algoritmo 1 (Algoritmo de Buchberger). Sejam I um ideal de $\mathbb{C}[\boldsymbol{x}]$ e $S \subset I$ finito tal $q u e\langle S\rangle=I$. Uma maneira de calcular uma Base de Gröbner $G$ a partir de $S$, é:

1. faça $G:=S$ e defina $H=\left\{\left(g, g^{\prime}\right) \mid g, g^{\prime} \in G, g \neq g^{\prime}\right\}$

2. Enquanto $H \neq \emptyset$, faça:

3. Escolha um par de polinômios $\left(g, g^{\prime}\right) \in H$ e remova-o de $H$

4. Calcule $r:=r_{G}\left(S\left(g, g^{\prime}\right)\right)$

5. Se $r \neq 0$, então faça $G=G \cup\{r\}$ e $H:=H \cup\{(g, r) \mid g \in G\}$

\section{Fim enquanto}

\section{Retorne $G$}

Note que, se em algum momento $H=\emptyset$, isso significa que o S-polinômio de todo par $\left(g, g^{\prime}\right) \in G$ deixa resto nulo na divisão por $G$ e, portanto, $G$ é uma Base de Gröbner; isto já justifica sua corretude. S-polinômios de elementos de $I$ também pertencem a $I$, assim como os restos de suas divisões por elementos de $I$; isso implica na finitude do algoritmo, quando somado ao fato de que $\mathbb{C}[\mathbf{x}]$ é um anel noetheriano, isto é, para toda cadeia de ideais crescente

$$
I_{0} \subseteq I_{1} \subseteq I_{2} \subseteq I_{3} \subseteq \ldots
$$

existe $k \in \mathbb{N}$ tal que $I_{j}=I_{k}$ para todo $j>k$ (condição da cadeia crescente).

Saber computar uma Base de Gröbner $G$ para $\langle P\rangle$ e o resto $r_{G}(1)$ é suficiente para decidir se o sistema $P=0$ tem ou não solução, porém é algo computacionalmente caro, uma vez que $G$ é um conjunto geralmente muito maior que $P$ e, muitas vezes, possui elementos redundantes. Para isso, define-se a Base de Gröbner reduzida de I, que além de única é, em certo sentido, a Base de Gröbner mais simples possível.

Definição 6 (Base de Gröbner Reduzida). Seja $I \subset \mathbb{C}[\boldsymbol{x}]$ um ideal e $G \subset I$ uma Base de Gröbner para I. G é reduzida quando:

1. Todo elemento de $G$ é mônico, isto é, $\mathrm{cl}(g)=1, \forall g \in G$;

2. Para quaisquer $g_{1}, g_{2} \in G$ distintos, $\mathrm{ml}\left(g_{1}\right) \nmid \mathrm{ml}\left(g_{2}\right)$;

3. Se $g \in G$, então para todo $h \in G \backslash\{g\}, r_{h}(g)=g$.

A partir de uma Base de Gröbner $G$, é possível reduzí-la removendo $g_{1}$ e introduzindo $r_{g_{1}}\left(g_{2}\right)$ em seu lugar sempre que $\mathrm{ml}\left(g_{1}\right)$ dividir $\mathrm{ml}\left(g_{2}\right)$ a fim de satisfazer a segunda propriedade; para satisfazer a terceira, basta trocar todo elemento de $G$ pelo seu resto na divisão por $G \backslash\{g\}$ (essas trocas ainda resultam em Bases de Gröbner). Esse processo pode ser visto como uma espécie de generalização da técnica de eliminação de Gauss para sistemas lineares. 
Exemplo 3. Seja $P=\left\{x^{2}-y z-3, y^{2}-x z-4, z^{2}-x y-5\right\} \subseteq \mathbb{C}[x, y, z]$ com a ordem lexicográfica, considerando $z \prec y \prec x$.

Sua Base de Gröbner reduzida, calculada pelo algoritmo de Buchberger e as trocas mencionadas acima, é

$$
G=\left\{x+\frac{11}{13} z, y-\frac{1}{13} z, z^{2}-\frac{56}{12}\right\} .
$$

É possivel visualizar a semelhança com a eliminação gaussiana notando que a equação $z^{2}-56 / 12=0$ depende apenas de z e que resolvê-la primeiro torna as outras duas também equações univariadas, o que é similar ao que ocorre num sistema triangular.

Para mais detalhes sobre sistemas polinomiais e vacuidade de conjuntos algébricos via Bases de Gröbner, veja [Cou09].

\subsection{Conjuntos semialgébricos e SOS}

A classe de conjuntos semialgébricos é ainda mais vasta que a de conjuntos algébricos (reais), pois são definidos por desigualdades polinomiais, ou seja, além dos exemplos anteriores, ainda englobam semiespaços, bolas e regiões externas e internas de curvas de nível fechadas e geradas por polinômios. A mesma motivação para estudar resultados sobre zeros de polinômios ao falar de conjuntos algébricos, se aplica a conjuntos semialgébricos, logo exibiremos algumas propriedades sobre polinômios não negativos, somas de quadrados e, novamente, critérios de infactiblidade, como o célebre positivstellensatz apresentado em 1974 por Gilbert Stengle (veja [Ste74]) e refinado posteriormente por Mihai Putinar (em [Put93]).

Definição 7 (Conjunto semialgébrico básico). Um conjunto $V \subseteq \mathbb{R}^{n}$ é dito semialgébrico básico se existem polinômios $p_{1}, \ldots, p_{v} \in \mathbb{R}[\boldsymbol{x}]$ tais que

$$
V=\left\{x \in \mathbb{R}^{n} \mid p_{i}(x) \square_{i} 0, \forall i \in\{1, \ldots, v\}\right\},
$$

onde $\square_{i} \in\{=, \neq,>, \geqslant\}$, isto é, se pode ser caracterizado por finitas igualdades e desigualdades polinomiais. Quando ele pode ser escrito de modo que $\square_{i}$ é igual a $\geqslant$ para todo $i$, ele é dito semialgébrico básico fechado.

É possível afirmar que interseções finitas e complementares de tais conjuntos ainda são semialgébricos básicos, o que não é verdade para uniões ou projeções, no geral. Por isso, definem-se conjuntos gerados por uniões finitas de conjuntos semialgébricos básicos (fechados) por conjuntos semialgébricos (fechados); estes são fechados quanto à projeção (resultado conhecido como teorema de Tarski-Seidenberg). Note que o conjunto $\mathcal{K}$ definido no Capítulo 2 é semialgébrico básico.

Todo conjunto semialgébrico básico pode ser definido por desigualdades na forma $p_{i}(x) \geqslant 0$ e uma única desigualdade $\neq$. Então, é razoável buscar propriedades úteis de polinômios que são não negativos em dadas regiões, o que é feito a seguir.

\subsubsection{Polinômios não negativos}

Um polinômio $p \in \mathbb{R}[\mathbf{x}]$ é dito ser não negativo em $X \subseteq \mathbb{R}^{n}$ quando $p(x) \geqslant 0$ para todo $x \in X$. Trata-se de um conceito bastante óbvio, mas o que desejamos é decidir se um dado polinômio é negativo ou não em $X$, e isso não é tão trivial. 


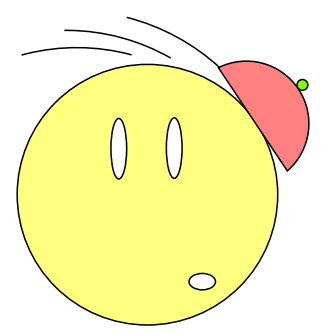

Figura 3.1: Um exemplo de conjunto semialgébrico, em que cada uma das regiões coloridas (e as linhas) são conjuntos semialgébricos básicos.

Exemplo 4. Se $\operatorname{deg}(p)=2$, então existem $A \in \mathcal{S}^{n}:=\left\{M \in \mathbb{R}^{n \times n} \mid M=M^{t}\right\}, b \in \mathbb{R}^{n} e$ $c \in \mathbb{R}$ tais que

$$
p(x)=x^{t} A x+2 b^{t} x+c .
$$

Logo, uma condição necessária e suficiente para que p seja não negativo é que

$$
M:=\left[\begin{array}{ll}
A & b \\
b^{t} & c
\end{array}\right]
$$

seja semidefinida positiva. De fato, se dados quaisquer $v \in \mathbb{R}^{n}$ e $u \in \mathbb{R}$, vale que

$$
q(s):=s^{t} M s=s^{t}\left(A v+u b, b^{t} v+u c\right)=v^{t} A v+2 u b^{t} v+u^{2} c \geqslant 0
$$

sendo $s:=(v, u) \in \mathbb{R}^{n+1}$, então, em particular, $q(x, 1)=p(x) \geqslant 0$ para todo $x \in \mathbb{R}^{n}$.

Por outro lado, se p é não negativo, então $p(0)=c \geqslant 0$ e A é semidefinida positiva, pois se houvesse algum $z \in \mathbb{R}^{n}$ tal que $z^{t} A z<0$, então teríamos

$$
p(\alpha z)=\alpha^{2} z^{t} A z+2 \alpha b^{t} z+c<0
$$

para algum $\alpha>0$ e isso seria um absurdo; logo $q(v, 0)=v^{t} A v \geqslant 0$ para todo $v \in \mathbb{R}^{n}$ e, para o caso restante, basta notar que

$$
q(v, u)=u^{2} p(v / u) \geqslant 0,
$$

para todo $(v, u)$ tal que $u \neq 0$.

Portanto, $q(s) \geqslant 0$ para todo $s \in \mathbb{R}^{n+1}$.

O Exemplo 4 sugere que há alguma relação entre polinômios quadráticos não negativos e matrizes semidefinidas positivas, o que de fato é verdade e será explorado nas seções seguintes.

Defina o conjunto de polinômios não negativos em $\mathbb{R}^{n}$ com grau até $2 d$ por

$$
\mathcal{P}_{n, 2 d}:=\left\{p \in \mathbb{R}[\mathbf{x}] \mid p(x) \geqslant 0, \forall x \in \mathbb{R}^{n} \text { e } \operatorname{deg}(p) \leqslant 2 d\right\},
$$

e defina também $\mathcal{P}_{n}:=\cup_{d \in \mathbb{N}} \mathcal{P}_{n, 2 d}$. É claro que todos os elementos de $\mathcal{P}_{n, 2 d}$ têm grau par, mas existem propriedades mais fortes a respeito desse conjunto, por exemplo:

Proposição 6. $\mathcal{P}_{n, 2 d}$ é um cone próprio.

Demonstração. Defina $N(d):=\left(\begin{array}{c}n+d \\ d\end{array}\right)$, que é o número total de monômios em $n$ variáveis com grau até $d$. O conjunto $\mathcal{P}_{n, 2 d}$ é isomorfo a algum $W \subseteq \mathbb{R}^{N(2 d)}$. Esse fato pode ser verificado escrevendo cada $p \in \mathcal{P}_{n, 2 d}$ como seu vetor de coeficientes $\vec{p}$. É elementar concluir que $\mathcal{P}_{n, 2 d}$ é um cone convexo fechado, pontudo e que qualquer polinômio que assume apenas valores estritamente positivos (identicamente constantes positivas, por exemplo) está em seu interior que, portanto, é não vazio. 
Mesmo conhecendo essa propriedade, $\mathcal{P}_{n, 2 d}$ ainda é algo substancialmente difícil de se explorar, pois foi provado que, para polinômios de grau maior que três, decidir se são não negativos em um dado conjunto ou não, é um problema $N P$-Hard (provável por meio de uma redução ao $\operatorname{lin} 3 S A T$, veja [Ahm17] para algo mais aprofundado).

\subsubsection{Somas de quadrados}

Existem muitas aplicações para o problema de decisão sobre a não negatividade de um polinômio em uma dada região, mas devido à grande dificuldade de lidar com ele, apelamos para uma alternativa mais fraca, porém razoavelmente mais amigável e fácil de manusear, que é buscar uma representação em somas de quadrados. Denote por $\mathbb{R}_{d}[\mathbf{x}]$ o conjunto dos polinômios em $n$ variáveis, com coeficientes em reais e grau menor ou igual a $d$.

Definição 8 (Soma de quadrados). Um polinômio $p \in \mathbb{R}_{2 d}[\boldsymbol{x}]$ é uma soma de quadrados (SOS) se existem $q_{1}, \ldots, q_{m} \in \mathbb{R}_{d}[\boldsymbol{x}]$ tais que

$$
p=\sum_{i=1}^{m} q_{i}^{2}
$$

Defina também $\Sigma_{n, 2 d}$ como o conjunto dos polinômios em $n$ variáveis tais que são somas de quadrados e têm grau menor ou igual a $2 d$. Além disso, $\Sigma_{n}:=\cup_{d \in \mathbb{N}} \Sigma_{n, 2 d}$.

Claramente, se um polinômio pode ser decomposto como soma de quadrados, então ele é não negativo. Porém, isso não é suficiente para caracterizar este conceito, já que a recíproca não é verdadeira. Em geral, decomposições em SOS não são únicas.

Proposição 7 . O conjunto $\Sigma_{n, 2 d}$ é um cone próprio em $\mathbb{R}_{2 d}[\boldsymbol{x}]$.

Demonstração. Completamente análoga à demonstração da Proposição 6.

Decidir se um polinômio é ou não soma de quadrados é, portanto, um problema de pertinência a um cone.

Proposição 8. Sejam $p=p_{1}^{2}+\cdots+p_{P}^{2}$ e $q=q_{1}^{2}+\cdots+q_{Q}^{2}$, ambos em $\Sigma_{n, 2 d}$, com $p_{1}, \ldots, p_{P}, q_{1}, \ldots, q_{Q} \in \mathbb{R}_{d}[\boldsymbol{x}]$.

1. $p+q \in \Sigma_{n, 2 d}$ e $p q \in \Sigma_{n, 4 d}$;

2. Se p e q são somas de apenas dois quadrados, então pq também o é;

3. Todo polinômio não negativo de $\mathbb{R}[x]$ pode ser decomposto como soma de dois quadrados;

Demonstração.

1. A soma é trivial e, para o produto, vale que

$$
p q=\sum_{(i, j)=(1,1)}^{(P, Q)} p_{i}^{2} q_{j}^{2}=\sum_{(i, j)=(1,1)}^{(P, Q)}\left(p_{i} q_{j}\right)^{2} .
$$

Além disso, como $\max \left\{\operatorname{deg}\left(p_{i}\right), \operatorname{deg}\left(q_{j}\right)\right\} \leqslant d$, então $\operatorname{deg}\left(p_{i} q_{j}\right) \leqslant 2 d, \operatorname{logo} p q \in \Sigma_{n, 4 d}$. 
2. Se $P=Q=2$, então

$$
\begin{gathered}
p q=\left(p_{1} q_{1}\right)^{2}+\left(p_{1} q_{2}\right)^{2}+\left(p_{2} q_{1}\right)^{2}+\left(p_{2} q_{2}\right)^{2} \\
p q=\left(p_{1} q_{1}\right)^{2}+\left(p_{2} q_{2}\right)^{2}-2 p_{1} p_{2} q_{1} q_{2}+\left(p_{1} q_{2}\right)^{2}+\left(p_{2} q_{1}\right)^{2}+2 p_{1} p_{2} q_{1} q_{2} \\
p q=\left(p_{1} q_{1}+p_{2} q_{2}\right)^{2}+\left(p_{1} q_{2}-p_{2} q_{1}\right)^{2} .
\end{gathered}
$$

Logo $p q$ pode ser escrito como soma de apenas dois quadrados.

3. Seja $p \in \mathbb{R}[x]$ não negativo, de grau $2 d$ e recorra ao teorema fundamental da álgebra para afirmar que

$$
p=\alpha_{0}\left(x-r_{1}\right)^{\alpha_{1}} \ldots\left(x-r_{R}\right)^{\alpha_{R}} \prod_{j=1}^{C}\left[\left(x-z_{j}\right)\left(x-\overline{z_{j}}\right)\right]^{\beta_{j}},
$$

onde $r_{1}, \ldots, r_{R} \in \mathbb{R}$ e $z_{1}, \overline{z_{1}}, \ldots, z_{C}, \overline{z_{C}} \in \mathbb{C}$ são todas as raízes de $p$, com $R+$ $2 C=2 d, \alpha_{0}>0$ é o coeficiente do termo de maior grau e $\alpha_{i}, \beta_{j} \in \mathbb{N}^{\star},(i, j) \in$ $\{1, \ldots, R\} \times\{1, \ldots, C\}$ são as multiplicidades das raízes. Note que as raízes reais têm multiplicidade par, pois se algum $\alpha_{i}$ fosse ímpar, então $\left(x-r_{i}\right)\left(x-r_{i}\right)^{\alpha_{i}-1}<0$ para todo $x<r_{i}$. Agora escreva $k:=\sqrt{\alpha_{0}}\left(x-r_{1}\right)^{\alpha_{1} / 2} \ldots\left(x-r_{R}\right)^{\alpha_{R} / 2}$ e note que

$$
\begin{gathered}
\left(x-z_{j}\right)\left(x-\overline{z_{j}}\right)=x^{2}-x\left(z_{j}+\overline{z_{j}}\right)+z_{j} \overline{z_{j}}= \\
=x^{2}-2 x \operatorname{Re}\left(z_{j}\right)+\operatorname{Re}\left(z_{j}\right)^{2}+\operatorname{Im}\left(z_{j}\right)^{2}=\left(x-\operatorname{Re}\left(z_{j}\right)\right)^{2}+\operatorname{Im}\left(z_{j}\right)^{2},
\end{gathered}
$$

ou seja, é soma de dois quadrados em $\mathbb{R}[x]$ para todo $j \in\{1, \ldots, C\}$. Pelo item anterior e dois argumentos indutivos diretos - um sobre a multiplicidade e outro sobre $j$ - é possível concluir que

$$
f:=\prod_{j=1}^{C}\left[\left(x-z_{j}\right)\left(x-\overline{z_{j}}\right)\right]^{\beta_{j}}
$$

é também soma de dois quadrados, suponha no caso, $f=a^{2}+b^{2}$. Desta forma

$$
p=k^{2}\left(a^{2}+b^{2}\right)=(k a)^{2}+(k b)^{2} ;
$$

portanto é possível concluir que $p$ é soma de apenas dois quadrados.

A Proposição 8 nos mostra que, no caso univariado, não negatividade e ser soma de quadrados são conceitos equivalentes. Além disso, Hilbert mostrou (em [Hil93]) que existem apenas mais dois casos onde isso é válido: quando tratamos de polinômios quadráticos em $n$ variáveis e quárticos em duas variáveis.

Teorema 9 (Hilbert). Existe a inclusão $\Sigma_{n, 2 d} \subseteq \mathcal{P}_{n, 2 d}$ se, e somente se, um dos três casos abaixo ocorre:

1. $n=1$;

2. $d=1$;

3. $n=d=2$. 
Por outro lado, um famoso exemplo de polinômio não negativo que não pode ser escrito como soma de quadrados é devido a Motzkin (veja [Mot67]).

Exemplo 5 (Motzkin). O polinômio $M(x, y):=x^{4} y^{2}+x^{2} y^{4}-3 x^{2} y^{2}+1$ é não negativo, mas não é soma de quadrados.

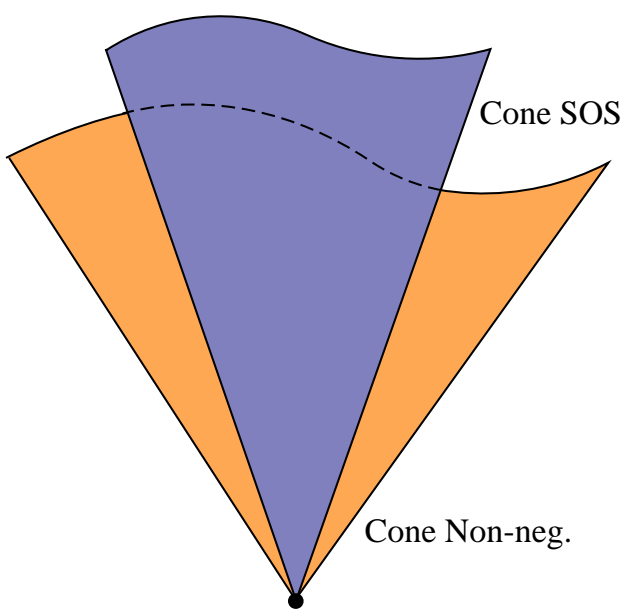

Figura 3.2: Representação de $\mathcal{P}_{n}$ e $\Sigma_{n}$.

Posteriormente, G. Blekherman mostrou que, conforme o grau $d$ se afasta de dois, a diferença entre $\mathcal{P}_{n, 2 d}$ e $\Sigma_{n, 2 d}$ torna-se mais significativa (maiores detalhes em [Ble06], inclusive uma medida explícita de crescimento).

As próximas duas seções tratam de aspectos mais práticos de somas de quadrados, isto é, como computar tal representação, se ela existir. Ilustraremos o procedimento com o caso de apenas uma variável e, em seguida, apresentaremos as devidas generalizações.

\subsubsection{Em uma variável}

É possível caracterizar um polinômio $p$ apenas por seu vetor ordenado (pelo grau) de coeficientes $\vec{p}:=\left(c_{i}\right)_{i \in \mathbb{N}}$, o que estabelece uma relação injetiva entre $\mathbb{R}[x]$ e $\mathbb{R}^{\infty}$. Analogamente, existe uma injetividade entre $\mathbb{R}_{2 d}[x]$ e $\mathbb{R}^{2 d+1}$ que leva $p$ no seu vetor de coeficientes truncado após a $(2 d+1)$-ésima componente, que denotaremos também por $\vec{p}$.

O que faremos a seguir é apresentar uma maneira conveniente de caracterizar polinômios SOS em uma variável. Ilustramos essa representação tomando $p \in \mathbb{R}_{2 d}[x]$ tal que existem $q_{1}, \ldots, q_{m} \in \mathbb{R}_{d}[x]$ de modo que

$$
p=q_{1}^{2}+\cdots+q_{m}^{2},
$$

em outras palavras, uma soma de quadrados. Se $q:=\left(q_{1}, q_{2}, \ldots, q_{m}\right)^{t}$ é um vetor de $\mathbb{R}_{d}[x]^{m}$, então $p=q^{t} q$. E mais: a correspondência descrita no parágrafo anterior nos permite construir uma matriz $V \in \mathbb{R}^{m \times(d+1)}$ cuja $i$-ésima linha é o vetor $\vec{q}_{i}^{t}$ e, com isso em mente, note que $q=V[x]_{d}$, onde

$$
[x]_{d}:=\left(1, x, \ldots, x^{d}\right)^{t} \in \mathbb{R}[x]^{d+1}
$$

é o vetor de monômios na variável $x$ e grau até $d$, ordenado da mesma maneira que o vetor de coeficientes. Juntando as pontas, verificamos que $p=[x]_{d}^{t} V^{t} V[x]_{d}$. Além disso, $V^{t} V$ é semidefinida positiva, pois dado qualquer $u \in \mathbb{R}^{d+1}$, vale que $u^{t} V^{t} V u=\|V u\|_{2}^{2} \geqslant 0$. Isso motiva a seguinte proposição: 
Teorema 10. Um polinômio $p \in \mathbb{R}_{2 d}[x]$ é soma de quadrados se, e somente se, existe $Q \in \mathcal{S}^{d+1}$ semidefinida positiva, tal que $p(x)=[x]_{d}^{t} Q[x]_{d}$.

Demonstração. O raciocínio anterior prova uma das implicações, com $Q=V^{t} V$.

Por outro lado, se existe $Q$ como enunciado, seja $L \in \mathbb{R}^{(d+1) \times(d+1)}$ a matriz do item 4 do Teorema 1 para $Q$, isto é, $Q=L^{t} L$. Daí

$$
p(x)=[x]_{d}^{t} Q[x]_{d}=[x]_{d}^{t} L^{t} L[x]_{d}=\|l(x)\|_{2}^{2}=\sum_{i=1}^{d+1} l_{i}(x)^{2},
$$

sendo $l(x)=\left(l_{1}(x), \ldots, l_{d+1}(x)\right)^{t}=L[x]_{d}$; isso mostra que $p$ é soma de quadrados e conclui a demonstração do teorema.

Essa representação é denominada representação de Gram e a matriz $Q$ é chamada de matriz de Gram. Pelo menos no caso univariado, é fácil explicitar cada coeficiente de $[x]_{d}^{t} Q[x]_{d}$ em termos das entradas $Q_{i, j}$ de $Q$; a fim de elucidar:

$$
[x]_{d}^{t} Q[x]_{d}=\sum_{i=0}^{d} \sum_{j=0}^{d} Q_{i j} x^{i+j}=\sum_{k=0}^{2 d}\left(\sum_{i+j=k} Q_{i j}\right) x^{k} .
$$

Portanto, o Teorema 10 reduz o problema de decisão sobre a não negatividade de $p(x):=$ $\sum_{k=0}^{2 d} p_{k} x^{k}$ a uma decisão sobre a existência de alguma matriz semidefinida positiva cujas entradas satisfazem um sistema linear de $2 d+1$ equações (uma matriz na interseção entre um conjunto algébrico e um cone). Para constar, eis o referido sistema:

$$
\left\{\begin{array}{r}
p_{k}=\sum_{i+j=k} Q_{i j}, \quad k=0, \ldots, 2 d \\
Q \succeq 0 .
\end{array}\right.
$$

Exemplo 6. Para expressar o polinômio $p(x)=x^{4}+4 x^{3}+6 x^{2}+4 x+5$ por meio de sua matriz de Gram, é necessário resolver o seguinte sistema:

$$
\left\{\begin{array}{l}
5=Q_{00} \\
4=Q_{10}+Q_{01}=2 Q_{10} \\
6=Q_{20}+Q_{02}+Q_{11}=2 Q_{20}+Q_{11} \\
4=Q_{21}+Q_{12}=2 Q_{21} \\
1=Q_{22} \\
Q \succeq 0
\end{array}\right.
$$

que tem como solução

$$
Q=\left[\begin{array}{lll}
5 & 2 & 0 \\
2 & 6 & 2 \\
0 & 2 & 1
\end{array}\right]=L^{t} L, \quad L=\left[\begin{array}{ccc}
0 & 2 & 1 \\
\sqrt{2} & \sqrt{2} & 0 \\
\sqrt{3} & 0 & 0
\end{array}\right]
$$

dai

$$
p(x)=\sum_{i=1}^{3} l_{i}(x)^{2}=\left(2 x+x^{2}\right)^{2}+(\sqrt{2}+\sqrt{2} x)^{2}+(\sqrt{3})^{2},
$$

é sua decomposição $S O S$. 
A relação entre polinômios não negativos em $\mathbb{R}$ e somas de quadrados é de igualdade (Proposição 8), mas não se limita a isso, pois existem formas de se caracterizar não negatividade em intervalos por meio de SOS; e isso é, inclusive, algo ainda mais interessante que na reta toda.

Proposição 11. Seja $p \in \mathbb{R}[x]$ tal que $\operatorname{deg}(p)=d$.

1. Sejam $g(x):=1-x^{2}$ e $I:=[-1,1]$, então $p \geqslant 0$ em $I$ se, e somente se, existem polinômios $f, h \in \Sigma_{1}$ tais que

$$
p=f+g h
$$

e o grau de cada um dos termos é menor que $2 d$.

2. Sejam $g_{1}(x):=1-x, g_{2}(x):=1+x$, então $p \geqslant 0$ em I se, e somente se, existem $f_{0}, f_{1}, f_{2}, f_{3} \in \Sigma_{1}$ tais que

$$
p=f_{0}+f_{1} g_{1}+f_{2} g_{2}+f_{3} g_{1} g_{2},
$$

além disso, o grau de cada um dos termos é menor ou igual a d e, se d é par, $f_{1}=f_{2}=0$ e, se d é ímpar, $f_{0}=f_{3}=0$.

A ideia principal por trás da prova do primeiro item da Proposição 11 é mapear $[-1,1]$ em $[0, \pi]$ fazendo a mudança de variável $x=\cos \theta$ e $\operatorname{considerar} p(\cos \theta)$, que é um cos-polinômio; sendo assim, um resultado de F. Riesz e L. Fejér (veja [FR16]) afirma que todo cos-polinômio não negativo pode ser escrito como $p(\cos \theta)=\left|h\left(e^{i \theta}\right)\right|^{2}$, sendo $h$ um polinômio com coeficientes reais. Mas como $e^{i \theta}=\cos \theta+i \sin \theta$, existem polinômios $A, B \in \mathbb{R}[x]$ tais que $h\left(e^{i \theta}\right)=A(\cos \theta)+i \sin \theta B(\cos \theta), \log 0$

$$
p(\cos \theta)=|A(\cos \theta)+i \sin \theta B(\cos \theta)|^{2} .
$$

Então, no primeiro item, por exemplo, teremos

$$
p(\cos \theta)=A(\cos \theta)^{2}+\sin ^{2} \theta B(\cos \theta)^{2}
$$

e o segundo item é similar. Esses resultados são devidos a M. Fekete e F. Lukàcs, respectivamente, e mais detalhes a respeito podem ser encontrados em [PS98].

Por fim, apresentamos um resultado devido a G. Polyá e G. Szegö em [PS98], que lida com a não negatividade no intervalo $[0, \infty)$.

Proposição 12. Seja $p \in \mathbb{R}[x]$ não negativo em $[0, \infty)$ com grau d. Então existem $f_{0}, f_{1} \in$ $\Sigma_{1, d}$ tais que

$$
p=f_{0}+x f_{1}
$$

e o grau de ambos os termos é, no máximo, d.

Nas Proposições 11 e 12, note a relação entre as caracterizações semialgébricas do intervalo $I$ e a não negatividade de $p$ nele, por meio dos polinômios que o definem. Por exemplo, os critérios de não negatividade em $[-1,1]=\{x \in \mathbb{R} \mid 1-x \geqslant 0,1+x \geqslant 0\}=$ $\left\{x \in \mathbb{R} \mid 1-x^{2} \geqslant 0\right\}$ podem depender tanto dos polinômios $1-x$ e $1+x$ quanto de $1-x^{2}$ e todas as representações envolvem o produto destes polinômios por somas de quadrados.

Maiores detalhes podem ser encontrados em [PBT13], [PR00] e [Las10]. 


\subsubsection{Em várias variáveis}

Os conceitos vistos para somas de quadrados em uma variável podem ser generalizados em termos de dimensão. Porém, também foi visto que não existe a equivalência entre ser SOS e não negatividade, no caso geral. Baseado na subseção anterior, considere o vetor de monômios ordenados lexico-graduadamente

$$
[\mathbf{x}]_{d}^{t}:=\left(1, x_{1}, x_{2}, \ldots, x_{n}, x_{1} x_{2}, \ldots, x_{n}^{d}\right) .
$$

Recordamos que um polinômio arbitrário de grau $d$ pode ter, no máximo, $N(d):=$ $\left(\begin{array}{c}n+d \\ d\end{array}\right)$ coeficientes não nulos. Logo, a caracterização vetorial de um polinômio $p \in \mathbb{R}_{2 d}[\mathbf{x}]$ é um elemento $\vec{p}$ de $\mathbb{R}^{N(2 d)}$, cujas componentes são os coeficientes de $p$ ordenados segundo a ordem induzida da monomial, isto é, de modo que $[\mathbf{x}]_{2 d}^{t} \vec{p}=p$. A passagem sobre polinômios de uma variável nos sugere que é possível encontrar uma representação de Gram para polinômios SOS em mais variáveis, uma vez que somente sua representação vetorial é importante e isso nada mais é do que apenas um caso de dimensões maiores que o primeiro, com raciocínios completamente análogos.

Teorema 13. Fixe uma ordem monomial $\preceq$. Um polinômio $p \in \mathbb{R}[\boldsymbol{x}]$ de grau $2 d$ é soma de quadrados se, e somente se, existe uma matriz $Q \in \mathcal{S}^{N(d)}$ indexada por $\preceq$ e semidefinida positiva tal que $p=[\boldsymbol{x}]_{d}^{t} Q[\boldsymbol{x}]_{d}$.

A indexação das entradas de $Q$ é feita de maneira similar ao caso univariado, por pares de vetores $(\beta, \gamma)$, onde $\beta, \gamma \in\{0, \ldots, d\}^{n}$, cada posição representa uma variável e cada entrada representa o grau desta variável. Por exemplo, a entrada de $Q$ associada aos monômios bivariados $x^{2}$ na linha e $x y$ na coluna será $Q_{20,11}$.

Da mesma forma, embora seja algo menos trivial de se perceber à primeira vista,

$$
[\mathbf{x}]_{d}^{t} Q[\mathbf{x}]_{d}=\sum_{\alpha \in\{0, \ldots, 2 d\}^{n}}\left(\sum_{\beta+\gamma=\alpha} Q_{\beta, \gamma}\right) \mathbf{x}^{\alpha}
$$

e isso representa o polinômio $p(x)=\sum_{\alpha \in\{0, \ldots, 2 d\}^{n}} p_{\alpha} \mathbf{x}^{\alpha}$ quando são satisfeitas as igualdades

$$
p_{\alpha}=\sum_{\beta+\gamma=\alpha} Q_{\beta, \gamma}
$$

totalizando $N(2 d)$ equações lineares e uma desigualdade matricial $Q \succeq 0$.

Exemplo 7. Considere o polinômio $p(x, y)=2 x^{4}+5 y^{4}-x^{2} y^{2}+2 x^{3} y+2 x+2$. Decidiremos se p é SOS buscando uma representação de p por meio de sua matriz de Gram, isto é, na forma:

$$
p(x, y)=[\boldsymbol{x}]_{d}^{t} G[\boldsymbol{x}]_{d}=\left[\begin{array}{c}
1 \\
x \\
y \\
x^{2} \\
x y \\
y^{2}
\end{array}\right]^{t}\left[\begin{array}{llllll}
Q_{00,00} & Q_{00,10} & Q_{00,01} & Q_{00,20} & Q_{00,11} & Q_{00,02} \\
Q_{10,00} & Q_{10,10} & Q_{10,01} & Q_{10,20} & Q_{10,11} & Q_{10,02} \\
Q_{01,00} & Q_{01,10} & Q_{01,01} & Q_{01,20} & Q_{01,11} & Q_{01,02} \\
Q_{20,00} & Q_{20,10} & Q_{20,01} & Q_{20,20} & Q_{20,11} & Q_{20,02} \\
Q_{11,00} & Q_{11,10} & Q_{11,01} & Q_{11,20} & Q_{11,11} & Q_{11,02} \\
Q_{02,00} & Q_{02,10} & Q_{02,01} & Q_{02,20} & Q_{02,11} & Q_{02,02}
\end{array}\right]\left[\begin{array}{c}
1 \\
x \\
y \\
x^{2} \\
x y \\
y^{2}
\end{array}\right]
$$

com $Q_{\beta, \gamma}=Q_{\gamma, \beta}$, pois $Q$ é simétrica.

Prosseguindo agora da mesma maneira que no Exemplo 6, desejamos encontrar as entradas de $Q$ que representem $p$, isto é, que satisfaçam o sistema linear de 15 equações abaixo (se ele tiver solução): 


$$
\left\{\begin{array}{l}
p_{00}=2=Q_{00,00} \\
p_{10}=2=2 Q_{00,10} \\
p_{01}=0=2 Q_{00,01} \\
p_{20}=0=2 Q_{00,20}+Q_{10,10} \\
p_{11}=0=2 Q_{10,01}+2 Q_{00,11} \\
p_{02}=0=2 Q_{00,02}+Q_{01,01} \\
p_{30}=0=2 Q_{10,20} \\
p_{21}=0=2 Q_{20,01}+2 Q_{11,10} \\
p_{12}=0=2 Q_{10,02}+2 Q_{11,01} \\
p_{03}=0=2 Q_{01,02} \\
p_{40}=2=Q_{20,20} \\
p_{31}=2=2 Q_{11,20} \\
p_{22}=-1=2 Q_{20,02}+Q_{11,11} \\
p_{13}=0=2 Q_{11,02} \\
p_{04}=5=Q_{02,02}
\end{array}\right.
$$

Intersectando o conjunto solução deste sistema com o cone das matrizes semidefinidas positivas, isto é, acrescentando a desigualdade $Q \succeq 0$, temos uma solução

$$
Q=\frac{1}{3}\left[\begin{array}{cccccc}
6 & 3 & 0 & -2 & 0 & -2 \\
3 & 4 & 0 & 0 & 0 & 0 \\
0 & 0 & 4 & 0 & 0 & 0 \\
-2 & 0 & 0 & 6 & 3 & -4 \\
0 & 0 & 0 & 3 & 5 & 0 \\
-2 & 0 & 0 & -4 & 0 & 15
\end{array}\right]
$$

e qualquer decomposição $L^{t} L$ de $Q$ produzirá uma representação em $S O S$ da forma $p(\boldsymbol{x})=$ $\sum_{i=1}^{15} l_{i}(\boldsymbol{x})^{2}$, onde $l_{i}(\boldsymbol{x})=L_{i} \cdot[\boldsymbol{x}]_{d}$ e $L_{i}$ é a i-ésima linha de $L$.

Os resultados vistos aqui fornecem uma forma de lidar com polinômios em $\Sigma_{n, 2 d}$ utilizando sistemas de equações lineares e desigualdades com variáveis matriciais. Tais problemas fazem parte de uma classe muito mais abrangente que será vista com mais detalhes no capítulo seguinte, chamada programação semidefinida.

A ligação entre a vacuidade de conjuntos semialgébricos e a não negatividade de algum polinômio neles é muito forte, afinal $p \geqslant 0$ num semialgébrico $\mathcal{K}$ se, e somente se, $\mathcal{K} \cap\{x \in$ $\left.\mathbb{R}^{n} \mid p(x)<0\right\}=\emptyset$. Logo, os resultados da última parte da subseção anterior serão generalizados na seção seguinte.

\subsection{Critérios de inviabilidade}

No problema de cobertura, o desafio é decidir se um dado conjunto semialgébrico $\mathcal{K} \subseteq \mathbb{R}^{n}$ é ou não vazio. Isso é algo que pode ser feito, para o caso particular dos conjuntos algébricos, com a ajuda das Bases de Gröbner e o Nullstellensatz de Hilbert (Seção 3.1.1). Além disso, as Proposições 11 e 12 fornecem os primeiros vislumbres sobre critérios de inviabilidade de conjuntos semialgébricos. Apresentamos, agora, uma espécie de generalização dessas ideias, majoritariamente em termos de dimensão.

Uma observação importante e não trivial a ser feita é que este problema é, de fato, decidível [PBT13]. 


\subsubsection{Estruturação}

Tratando de equações polinomiais, toda combinação entre seus elementos adicionada ao sistema é redundante. Mas, às vezes, algumas delas são mais convenientes para encontrar uma solução do que as próprias equações originais (Exemplo 3). Com desigualdades polinomiais, redundâncias podem ser geradas fazendo combinações com polinômios SOS, o que torna interessante trabalhar com uma estrutura que represente tais casos, assim como o ideal faz com as igualdades; esta é exatamente a noção de módulo quadrático.

Definição 9 (Módulo quadrático). Dados polinômios em $P=\left\{p_{1}, \ldots, p_{m}\right\} \subset \mathbb{R}[\boldsymbol{x}]$, define-se o módulo quadrático gerado por $P$ como o conjunto

$$
\text { qmodulo }(P):=\left\{q_{0}+p_{1} q_{1}+\cdots+p_{m} q_{m} \mid q_{i} \in \Sigma_{n}, \forall i \in\{0, \ldots, m\}\right\} \text {. }
$$

Veja que, como $P \subset$ qmodulo $(P)$, o subconjunto de $\mathbb{R}^{n}$ onde $P$ é não negativo, denotado por

$$
\text { nonneg }(P):=\left\{x \in \mathbb{R}^{n} \mid p(x) \geqslant 0, \forall p \in P\right\},
$$

está contido no conjunto

$$
\text { nonneg }(\operatorname{qmodulo}(P)):=\left\{x \in \mathbb{R}^{n} \mid p(x) \geqslant 0, \forall p \in \operatorname{qmodulo}(P)\right\}
$$

e isso é o que dá sentido ao uso da palavra "redundância" anteriormente. Faz-se necessário mencionar que para todo módulo quadrático $Q \subset \mathbb{R}[\mathbf{x}]$ vale que $1 \in Q, Q+Q \subset Q$ e $\Sigma_{n} Q \subset Q$; disso segue que $\Sigma_{n} \subset Q$ e que $-1 \in Q$ se, e somente se, $Q=\mathbb{R}[\mathbf{x}]$ (isto é, se $Q$ não é próprio).

Da mesma forma, nonneg(qmodulo $(P))=\operatorname{nonneg}\left(\operatorname{qmodulo}\left(P \cup\left\{p_{i} p_{j}\right\}_{i \neq j}\right)\right)$ e isso serve de justificativa para considerar um conjunto de redundâncias ainda maior.

Definição 10 (Pré-ordem). Dados polinômios em $P=\left\{p_{1}, \ldots, p_{m}\right\} \subset \mathbb{R}[\boldsymbol{x}]$, define-se a pré-ordem gerada por $P$ como o conjunto

$$
\operatorname{preorder}(P):=\left\{q_{0}+\sum_{i} p_{i} q_{i}+\sum_{i, j} p_{i} p_{j} q_{i j}+\sum_{i, j, k} p_{i} p_{j} p_{k} q_{i j k}+\ldots\right\}
$$

onde cada um dos $2^{m}$ termos é livre de quadrados e os polinômios $q_{i}$, $q_{i j}$ etc. são somas de quadrados; ou, de forma sucinta,

$$
\operatorname{preorder}(P)=\left\{q_{0}+\sum_{\emptyset \neq \Lambda \subset\{1, \ldots, m\}}\left(q_{\Lambda} \prod_{i \in \Lambda} p_{i}\right) \mid q_{\Lambda} \in \Sigma_{n}, \forall \emptyset \neq \Lambda \subset\{1, \ldots, m\}\right\}
$$

De fato, assim como os módulos quadráticos, as pré-ordens geradas por $P$ são cones convexos cujos elementos são não negativos em nonneg $(P)$.

Definição 11. Dados $f_{1}, \ldots, f_{r} \in \mathbb{R}[\boldsymbol{x}]$, defina o conjunto

$$
\operatorname{monoid}\left(f_{1}, \ldots, f_{r}\right):=\left\{f_{1}^{b_{1}} \ldots f_{r}^{b_{r}} \mid b_{1}, \ldots, b_{r} \in \mathbb{N}\right\}
$$

Este conjunto é um monóide multiplicativo.

Posicionados os jogadores, iniciaremos apresentando um critério de inviabilidade para conjuntos semialgébricos lineares, para depois ligar essas ideias ao caso polinomial. 


\subsubsection{O caso linear}

Vejamos o caso simples, no qual temos um sistema de equações lineares complexas, que pode ser expresso matricialmente como $A x+b=0$, sendo $A \in \mathbb{C}^{m \times n}, x \in \mathbb{C}^{n} \mathrm{e}$ $b \in \mathbb{C}^{m}$. A existência de um vetor $\mu \in \mathbb{C}^{m}$, tal que $\mu(A x+b) \neq 0$ para todo $x$, é uma condição suficiente (e trivial) para inviabilidade. Mais do que isso:

Teorema 14 (Alternativa de Fredholm). Dado um sistema linear complexo $A x+b=0$, com $A \in \mathbb{C}^{m \times n}$ e $b \in \mathbb{C}^{m}$, ele é infactivel se, e somente se, existe $\mu \in \mathbb{C}^{m}$ tal que $A^{t} \mu=0$ e $\mu^{t} b=-1$.

Seja $a_{i}$ a $i$-ésima linha de $A$, de modo que a solução do sistema é o conjunto algébrico $F=\left\{x \in \mathbb{C}^{n} \mid a_{1} x+b_{1}=0, \ldots, a_{m} x+b_{m}=0\right\}$. Então, o Teorema 14 afirma que $F$ é vazio se, e somente se, existe uma combinação linear das funções $x \mapsto a_{i} x$ que resulta no polinômio constante $x \mapsto 1$; isso claramente se assemelha a um caso particular do Nullstellensatz, com hipóteses e conclusões mais fortes. Os escalares $\mu_{i}$ do Teorema 14 são chamados certificados de inviabilidade para o sistema $A x+b=0$. Computar esses certificados consiste em resolver um sistema linear, por exemplo, utilizando eliminação gaussiana, que é um "caso particular" do método das Bases de Gröbner apresentado na Seção 3.1.1.

Agora, tratando de desigualdades e sobre o corpo ordenado real, o Lema de Farkas melhora este resultado, em certos aspectos.

Teorema 15 (Lema de Farkas). Dados $A \in \mathbb{R}^{m \times n}, b \in \mathbb{R}^{m}, C \in \mathbb{R}^{k \times n}$ e $d \in \mathbb{R}^{k}$, defina a relação $\leqslant$ para vetores induzida da ordenação em $\mathbb{R}$ coordenada a coordenada. Nestas condições o sistema:

$$
\begin{aligned}
& A x+b=0, \\
& C x+d \geqslant 0
\end{aligned}
$$

é infactível se, e somente se, existem $\mu \in \mathbb{R}^{m}$ e $0 \leqslant \eta \in \mathbb{R}^{k}$ tais que

$$
\begin{aligned}
& A^{t} \mu+C^{t} \eta=0, \\
& b^{t} \mu+d^{t} \eta=-1 .
\end{aligned}
$$

Note que $\mu^{t} A x$ pertence ao ideal $I=\left\langle a_{1} x, \ldots, a_{m} x\right\rangle$ e $\eta^{t} C x$ é um elemento do conjunto $Q=$ qmodulo $\left(c_{i} x, \ldots, c_{k} x\right)$, ou seja, se o conjunto semialgébrico formado por $A, b, C$ e $d$ é vazio, então o Teorema 15 afirma que $-1 \in I+Q$. Para computar esses certificados, basta resolver o problema de programação linear (PL) abaixo:

$$
\begin{gathered}
\min b^{t} \mu+d^{t} \eta+1 \\
\text { sujeito a } \\
A^{t} \mu+C^{t} \eta=0 \\
b^{t} \mu+d^{t} \eta+1 \geqslant 0 \\
\eta \geqslant 0 .
\end{gathered}
$$

Na próxima seção, será apresentada uma generalização desse resultado envolvendo somas de quadrados de polinômios, o que sugere a presença de programação semidefinida (que generaliza a programação linear, mais detalhes na Seção 4.1) na abordagem computacional, devido ao que foi visto na seção anterior. 


\subsubsection{O caso polinomial}

Assim como o weak nullstellensatz pode ser visto como uma generalização do Teorema 14 para polinômios quaisquer em $\mathbb{C}[\mathbf{x}]$, é possível generalizar o Lema de Farkas para polinômios reais.

Teorema 16 (Positivstellensatz (Stengle)). Dados polinômios $f_{i}, g_{j}, h_{k} \in \mathbb{R}[\boldsymbol{x}]$, com os indices $(i, j, k) \in \Lambda:=\{1, \ldots, r\} \times\{1, \ldots, s\} \times\{1, \ldots, t\}$, o conjunto

$$
\mathcal{K}:=\left\{x \in \mathbb{R}^{n} \mid f_{i}(x) \neq 0, g_{j}(x) \geqslant 0, h_{k}(x)=0\right\}
$$

é vazio se, e somente se, existem $F \in \operatorname{monoid}\left(f_{1}, \ldots, f_{r}\right), G \in \operatorname{preorder}\left(g_{1}, \ldots, g_{s}\right) e$ $H \in\left\langle h_{1}, \ldots, h_{t}\right\rangle$ tais que

$$
F^{2}+G+H=0
$$

Este teorema (veja mais em [Ste74]) é uma das peças centrais da geometria algébrica real contemporânea, devido à sua imensa versatilidade. Abreviadamente, este teorema será chamado P-satz e, decorrentes dele:

Corolário 17. Sejam $g_{1}, \ldots, g_{m}, h_{1}, \ldots, h_{k} \in \mathbb{R}[\boldsymbol{x}]$.

1. O conjunto

$$
\mathcal{K}:=\left\{x \in \mathbb{R}^{n} \mid h_{i}(x)=0, g_{j}(x) \geqslant 0,(i, j) \in\{1, \ldots, t\} \times\{1, \ldots, s\}\right\}
$$

é vazio se, e somente se, existem $H \in\left\langle h_{1}, \ldots, h_{t}\right\rangle$ e $G \in \operatorname{preorder}\left(g_{1}, \ldots, g_{s}\right)$ tais que

$$
H+G=-1 .
$$

2. O conjunto

$$
\mathcal{K}:=\left\{x \in \mathbb{R}^{n} \mid f_{i}(x) \neq 0, g_{j}(x) \geqslant 0,(i, j) \in\{1, \ldots, r\} \times\{1, \ldots, s\}\right\}
$$

é vazio se, e somente se, existem $F \in \operatorname{monoid}\left(f_{1}, \ldots, f_{r}\right)$ e $G \in \operatorname{preorder}\left(g_{1}, \ldots, g_{s}\right)$ tais que

$$
F^{2}+G=0
$$

Demonstração.

1. Se existem tais polinômios, então para qualquer $x \in \mathcal{K}$, vale que $G(x)=-1$, mas o fato de $G$ em $\mathcal{K}$ ser uma combinação positiva de somas de quadrados gera uma contradição. A recíproca é direta, considerando $f \in \mathbb{R}[\mathbf{x}]$ tal que $f \equiv 1$ e aplicando o positivstellensatz ao conjunto $\mathcal{K}^{\star}:=\mathcal{K} \cap\left\{x \in \mathbb{R}^{n} \mid f(x) \neq 0\right\}$, pois obviamente $\mathcal{K}=\mathcal{K}^{\star}$ e monoid $(f)=\{1\}$.

2. Análogo ao primeiro item, mas aplicando o $P$-satz ao conjunto $\mathcal{K} \cap\left\{x \in \mathbb{R}^{n} \mid h(x)=\right.$ $0\}$, sendo $h \equiv 0$. Tenha em mente também que $0 \notin \operatorname{monoid}\left(f_{1}, \ldots, f_{r}\right)$.

O item 4 do corolário seguinte é um pequeno critério de inviabilidade para conjuntos semialgébricos básicos fechados. 
Corolário 18. Dados $p, g_{1}, \ldots, g_{m} \in \mathbb{R}[\boldsymbol{x}]$, defina $\mathcal{K}:=\left\{x \in \mathbb{R}^{n} \mid g_{i} \geqslant 0, \forall i \in\{1, \ldots, m\}\right\}$. São válidos:

1. $p>0$ em $\mathcal{K}$ se, e somente se, existem $f, g \in \operatorname{preorder}\left(g_{1}, \ldots, g_{m}\right)$ tais que $p f=1+g$;

2. $p \geqslant 0$ em $\mathcal{K}$ se, e somente se, existem $f, g \in \operatorname{preorder}\left(g_{1}, \ldots, g_{m}\right), k \in \mathbb{N}$ tais que $p f=p^{2 k}+g$

3. $p=0$ em $\mathcal{K}$ se, e somente se, $-p^{2 k} \in \operatorname{preorder}\left(g_{1}, \ldots, g_{m}\right)$ para algum $k \in \mathbb{N}$;

4. $\mathcal{K}=\emptyset$ se, e somente se, $-1 \in \operatorname{preorder}\left(g_{1}, \ldots, g_{m}\right)$.

Demonstração.

1. Se $p>0$ em $\mathcal{K}$, aplique o primeiro corolário do $P$-satz ao conjunto vazio

$$
\mathcal{K}_{1}:=\{x \in \mathcal{K} \mid-p(x) \geqslant 0\}
$$

reciprocamente, se existem tais polinômios, o fato de $g$ e $f$ serem não negativos em $\mathcal{K}$ implica que $1+g$ é positivo. Logo, $f$ é positivo.

2. Se $p \geqslant 0$ em $\mathcal{K}$, aplique o $P$-satz ao conjunto vazio

$$
\mathcal{K}_{2}:=\{x \in \mathcal{K} \mid p(x) \neq 0,-p(x) \geqslant 0\} ;
$$

a recíproca decorre da afirmação de que $f, g$ são não negativos.

3. Se $p=0$ em $\mathcal{K}$, aplique o $P$-satz ao conjunto vazio

$$
\mathcal{K}_{3}:=\{x \in \mathcal{K} \mid p(x) \neq 0\}
$$

e terá $g \in \operatorname{preorder}\left(g_{1}, \ldots, g_{m}\right)$ e $p^{k} \in \operatorname{monoid}(p):=\left\{p^{d} \mid d \in \mathbb{N}\right\}$ tais que $\left(p^{k}\right)^{2}+g=$ 0 . Para a recíproca, note que $-p^{2 k}$ é não positivo em $\mathcal{K}$. Logo, só pertence à préordem gerada pelos $g_{i}, i \in\{1, \ldots, m\}$ se for nulo em $\mathcal{K}$, o que implica que $p=0 \mathrm{em}$ $\mathcal{K}$.

4. Aplique o corolário do P-satz ao conjunto

$$
\mathcal{K}_{4}:=\{x \in \mathcal{K} \mid h(x)=0\},
$$

sendo $h \equiv 0$.

É possível agora enunciar formalmente uma versão real do weak nullstellensatz, como a discussão na Seção 3.1 sugere (também em [Ste74]).

Corolário 19 (Weak real nullstellensatz). Seja $P:=\left\{p_{1}, \ldots, p_{m}\right\} \subset \mathbb{R}[\boldsymbol{x}]$. Então, $Z(P)=$ $\emptyset$ se, e somente se, $-1 \in \Sigma_{n}+\langle P\rangle$.

Demonstração. Se existissem $q_{1}, \ldots, q_{m} \in \mathbb{C}[\mathbf{x}], s \in \Sigma_{n}$ e algum ponto $a \in Z(P)$, tais que $-1=s+\sum_{i=1}^{m} p_{i} q_{i}$, então $s(a)=-1$ e isto seria um absurdo. Por outro lado, aplicar o $P$-satz ao conjunto

$$
\emptyset=\left\{x \in \mathbb{R}^{n} \mid f(x):=1 \neq 0, g(x):=0 \geqslant 0, p_{1}(x)=0, \ldots, p_{m}(x)=0\right\}
$$

nos dá polinômios $1 \in \operatorname{monoid}(1), s \in \operatorname{preorder}(0)$ e $H \in\langle P\rangle$ tais que $1+s+H=0$, o que completa a demonstração. 


\subsubsection{Conjuntos compactos}

O primeiro item do Corolário 18 do positivstellensatz fornece uma caracterização de polinômios positivos em conjuntos semialgébricos. Agora, adicionaremos uma hipótese e nos restringiremos a estudar conjuntos semialgébricos compactos, o que nos permite obter resultados mais fortes, como representações em somas de quadrados mais simples. Note a semelhança entre os resultados seguintes e as Proposições 11 e 12.

Konrad Schmüdgen (em [Sch91]) propôs e demonstrou o resultado a seguir, que foi considerado um marco por ser mais simples que o Corolário 18 e não necessitar de hipóteses adicionais sobre o conjunto.

Teorema 20 (Positivstellensatz (Schmüdgen)). Dados $p, g_{1}, \ldots, g_{m} \in \mathbb{R}[\boldsymbol{x}]$, suponha que o conjunto $\mathcal{K}:=\left\{x \in \mathbb{R}^{n} \mid g_{i} \geqslant 0, \forall i \in\{1, \ldots, m\}\right\}$ é compacto. Nestas condições, se $p>0$ em $\mathcal{K}$, então $p \in \operatorname{preorder}\left(g_{1}, \ldots, g_{m}\right)$.

Uma representação em função da pré-ordem ainda possui "termos demais", cuja quantidade cresce exponencialmente com $m$. Então, Mihai Putinar possibilitou simplificá-la ainda mais (substituindo a pré-ordem pelo módulo quadrático), em troca de uma hipótese mais exigente.

Teorema 21. Dados $g_{1}, \ldots, g_{m} \in \mathbb{R}[\boldsymbol{x}]$ tais que $\mathcal{K}=\left\{x \in \mathbb{R}^{n} \mid g_{1} \geqslant 0, \ldots, g_{m} \geqslant 0\right\}$ é compacto, as seguintes afirmações são equivalentes:

1. Existe $f \in$ qmodulo $\left(g_{1}, \ldots, g_{m}\right)$ tal que o conjunto $\left\{x \in \mathbb{R}^{n} \mid f(x) \geqslant 0\right\}$ é compacto;

2. Existe $M \in \mathbb{N}$ tal que $M-\sum_{i=1}^{n} x_{i}^{2} \in$ qmodulo $\left(g_{1}, \ldots, g_{m}\right)$;

3. Para todo $p \in \mathbb{R}[\boldsymbol{x}]$ existe $M \in \mathbb{N}$ tal que $M+p$ ou $M-p$ pertencem a qmodulo $\left(g_{1}, \ldots, g_{m}\right)$;

4. Existem $p_{1}, \ldots, p_{s} \in \mathbb{R}[\boldsymbol{x}]$ tais que

$$
\left\{x \in \mathbb{R}^{n} \mid p_{i}(x) \geqslant 0, i \in\{1, \ldots, s\}\right\}
$$

é compacto $e \prod_{i \in \Lambda} p_{i} \in$ qmodulo $\left(g_{1}, \ldots, g_{m}\right)$, para todo $\Lambda \subset\{1, \ldots, s\}$.

Quando um módulo quadrático satisfaz qualquer um dos itens acima, ele é dito arquimediano e é exatamente essa a exigência adicional que, aliás, não é tão restritiva; ela é satisfeita, por exemplo, quando $\mathcal{K}$ é um polítopo ou quando $\left\{x \in \mathbb{R}^{n} \mid g_{j}(x) \geqslant 0\right\}$ é compacto, para algum $j \in\{1, \ldots, m\}$. Além disso, se $\mathcal{K} \subset B(0, M):=\left\{x \in \mathbb{R}^{n} \mid\|x\|_{2}^{2} \leqslant M\right\}$ para algum $M>0$, então o polinômio $g_{m+1}:=M-\|x\|_{2}^{2}$ é redundante a $\mathcal{K}$, isto é, $\mathcal{K}=\left\{x \in \mathbb{R}^{n} \mid g_{1}(x) \geqslant 0, \ldots, g_{m}(x) \geqslant 0, g_{m+1}(x) \geqslant 0\right\}$. Mas essa nova representação é arquimediana, pois $M-\|x\|_{2}^{2} \in$ qmodulo $\left(g_{1}, \ldots, g_{m+1}\right)$. Logo, todo conjunto compacto tem uma representação arquimediana.

Enunciemos formalmente o resultado de Putinar (mais detalhes em [Put93]), que utilizaremos extensivamente na resolução do problema de cobertura:

Teorema 22 (Positivstellensatz (Putinar)). Dados $p, g_{1}, \ldots, g_{m} \in \mathbb{R}[x]$, defina $\mathcal{K}:=\{x \in$ $\left.\mathbb{R}^{n} \mid g_{i} \geqslant 0, \forall i \in\{1, \ldots, m\}\right\}$ e suponha que qmodulo $\left(g_{1}, \ldots, g_{m}\right)$ é arquimediano. Se $p>0$ em $\mathcal{K}$, então $p \in$ qmodulo $\left(g_{1}, \ldots, g_{m}\right)$.

A demonstração do Teorema 22 (versão apresentada em [Las10]), apesar de elementar, é extensa, porém é necessário exibí-la em detalhes devido ao seu corolário. Mas, antes disso, seguem alguns lemas. 
Lema 1. Se $Q \subset \mathbb{R}[\boldsymbol{x}]$ é um módulo quadrático próprio maximal, isto é, se o único módulo quadrático próprio que contém $Q$, é $Q$, então $Q \cap(-Q)$ é um ideal e $Q \cup(-Q)=\mathbb{R}[\boldsymbol{x}]$.

Demonstração. Para a primeira parte, seja $I:=Q \cap(-Q)$. I é um ideal, pois se $a, c \in I$ e $b \in \mathbb{R}[\mathbf{x}]$, então

$$
a b=a(b+1)^{2} / 2+(-a)(b-1)^{2} / 2 \in(Q \cap(-Q)) \Sigma_{n}+((-Q) \cap Q) \Sigma \subset Q \cap(-Q)=I,
$$

usando as propriedades gerais de um módulo quadrático. Trivialmente $a+c \in I$.

Agora suponha que $Q \cup(-Q) \neq \mathbb{R}[\mathbf{x}]$. Então, existe $f \in \mathbb{R}[\mathbf{x}] \backslash(Q \cup(-Q))$ e tanto $Q+f \Sigma_{n}$ quanto $Q-f \Sigma_{n}$ contêm $Q$. Portanto, devido à maximalidade de $Q$, não são próprios, isto é, existem $g_{1}, g_{2} \in Q$ e $s_{1}, s_{2} \in \Sigma_{n}$ tais que $-1=g_{1}+f s_{1}$ e $-1=g_{2}-f s_{2}$. Multiplique a primeira igualdade por $s_{2}$, a segunda por $s_{1}$ e some-as para obter

$$
s_{1}+s_{2}+s_{1} g_{1}+s_{2} g_{2}=0 \text {. }
$$

Mas $s_{1}=-\left(s_{2}+s_{1} g_{1}+s_{2} g_{2}\right) \in-Q$ e similarmente, $s_{2} \in-Q$. Isso somado ao fato de que $\Sigma_{n} \subset Q$, nos permite concluir que $s_{1}, s_{2} \in I$, que é um ideal. Isso implica que $s_{1} f$ e $s_{2} f$ pertencem a $I$ e, portanto, $-1=g_{1}+s_{1} f \in Q$ e $1=g_{2}+\left(-s_{2} f\right) \in-Q$, cada uma destas duas afirmações é um absurdo, pois $Q$ é próprio.

Lema 2. Sejam $Q \subset \mathbb{R}[\boldsymbol{x}]$ um módulo quadrático maximal, próprio e arquimedeano, $I:=$ $Q \cap(-Q)$ e $f \in \mathbb{R}[\boldsymbol{x}]$. Então existe um único $a \in \mathbb{R}$ tal que $f-a \in I$.

Demonstração. Como $Q$ é arquimediano, então $A:=\{a \in \mathbb{R} \mid f-a \in Q\}$ e $B:=\{b \in \mathbb{R} \mid$ $b-f \in Q\}$ são não vazios, pelo item 3 do Teorema 21. Note que todo elemento $c \in A \cap B$ satisfaz $f-c \in Q$ e $f-c \in-Q$. Logo, basta mostrar que $A \cap B$ possui um único elemento.

Para quaisquer $a \in A$ e $b \in B$, temos que $a \leqslant b$, pois, se $b$ fosse menor que $a$, então $0>b-a=(b-f)+(f-a) \in Q$ e isto seria um absurdo; tomemos então $a_{0}:=\sup A$ e $b_{0}:=\inf B$ e notemos que se existisse $c \in \mathbb{R}$ tal que $a_{0}<c<b_{0}$, então $f-c$ e $c-f$ não poderiam estar em $Q$, isto é, $f-c \notin Q \cup(-Q)=\mathbb{R}[\mathbf{x}]$, o que seria um absurdo. Portanto, $a_{0}=b_{0}$. Resta-nos apenas mostrar que $a_{0} \in A$ e $b_{0} \in B$ para concluirmos que $A \cap B=\left\{a_{0}\right\}$.

Suponha que $a_{0} \notin A$, isto é, que $f-a_{0} \notin Q$. Então, $Q^{\prime}:=Q+\left(f-a_{0}\right) \Sigma_{n}$ contém $Q$ e, por isso, não pode ser próprio, uma vez que $Q$ é máximal. Logo, devem existir $s \in \Sigma_{n}$ e $g \in Q$ tais que $-1=g+\left(f-a_{0}\right) s$. Como $Q$ é arquimediano, existe $M \in \mathbb{N}$ tal que $M-s \in Q$ (Teorema 21, item 3) e $\varepsilon>0$ tal que $\varepsilon<M^{-1}$. Como $a_{0}-\varepsilon \in A$ (pois $a_{0}$ é o menor limitante superior para $A)$, temos que $f-\left(a_{0}-\varepsilon\right) \in Q$, consequentemente $-1+\varepsilon s=g+\left(f-\left(a_{0}-\varepsilon\right)\right) s \in Q$ e $\varepsilon(M-s) \in \Sigma_{n} Q \subset Q$. Somando tudo, concluímos que $0>\varepsilon M-1 \in Q$, o que é um absurdo. Portanto, $a_{0} \in A$. Similarmente, $b_{0} \in B$ e isto encerra a demonstração.

Lema 3. Seja $f \in \mathbb{R}[x]$ estritamente positivo em um conjunto $\mathcal{K}=\left\{x \in \mathbb{R}^{n} \mid g_{1}(x) \geqslant\right.$ $\left.0, \ldots, g_{m}(x) \geqslant 0\right\}$ tal que qmodulo $\left(g_{1}, \ldots, g_{m}\right)$ é arquimediano. Então, existe $s \in \Sigma_{n}$ tal que $s f \in 1+$ qmodulo $\left(g_{1}, \ldots, g_{m}\right)$. Além disso, existem $h \in$ qmodulo $\left(g_{1}, \ldots, g_{m}\right)$ e $M \in \mathbb{N}$ tais que $M-h \in \Sigma_{n}$ e $h f \in 1+$ qmodulo $\left(g_{1}, \ldots, g_{m}\right)$.

Demonstração. Seja $Q_{0}:=$ qmodulo $\left(g_{1}, \ldots, g_{m}\right)-f \Sigma_{n}$. Se $Q_{0}=\mathbb{R}[\mathbf{x}]$, então $-1 \in Q_{0}$, isto é, existem $s \in \Sigma_{n}$ e $G \in$ qmodulo $\left(g_{1}, \ldots, g_{m}\right)$ tais que $f s=1+G$. Por outro lado, se $Q_{0} \neq \mathbb{R}[\mathbf{x}]$, então existe um ideal maximal $Q$ contendo $Q_{0}$. Além disso, como $0-f \Sigma_{n} \subset Q_{0}$, segue que qmodulo $\left(g_{1}, \ldots, g_{m}\right) \subset Q$. Logo, $Q$ também é arquimedeano. 
Pelo Lema 2, existem únicos $a_{1}, \ldots, a_{n} \in \mathbb{R}$ tais que $x_{i}-a_{i} \in I:=Q \cap(-Q)$, para todo $i \in\{1, \ldots, n\}$. Defina $a:=\left(a_{1}, \ldots, a_{n}\right)$. Pelo algoritmo da divisão (Teorema 4 ), existem $q_{1}, \ldots, q_{n}, r_{q} \in \mathbb{R}[\mathbf{x}]$ tais que

$$
f=r_{q}+\sum_{i=1}^{n} q_{i}\left(x_{i}-a_{i}\right)
$$

e $\mathrm{ml}\left(r_{q}\right)$ não divide $\mathrm{ml}\left(x_{i}-a_{i}\right)$, para todo $i \in\left\{q_{1}, \ldots, q_{m}\right\}$, o que significa que $r_{q}$ é constante. Mais que isso: note que $f(a)=r_{q}$, então $f-f(a)=\sum_{i=1}^{n} q_{i}\left(x_{i}-a_{i}\right) \in I$. Isso vale para qualquer $f \in \mathbb{R}[\mathbf{x}]$, em particular, escolhendo $f:=g_{j}$, uma vez que $g_{j} \in$ qmodulo $\left(g_{1}, \ldots, g_{m}\right) \subset Q$ e $g_{j}-g_{j}(a) \in I \subset Q$, obtemos que $g_{j}(a)=g_{j}-\left(g_{j}-g_{j}(a)\right) \in Q$. Isso implica que $g_{j}(a) \geqslant 0$ para todo $j \in\{1, \ldots, m\}$, pois $Q$ é próprio. Logo, $a \in \mathcal{K}$. Quando $\mathcal{K}$ é vazio, temos um absurdo aqui e, portanto, concluímos que $Q_{0}$ não é próprio e a demonstração se encerra. Caso contrário, note que $-f \in Q_{0}$. Logo, $-f(a)=(f-$ $f(a))+(-f) \in Q$, o que implica que $-f(a) \geqslant 0$ e isto também é um absurdo, pois $a \in \mathcal{K}$ e $f$ é estritamente positivo em $\mathcal{K}$, por hipótese, logo $Q_{0}$ não pode ser próprio. Isto encerra a primeira parte da demonstração.

Para a segunda parte, considere $s \in \Sigma_{n}$ tal que $s f \in 1+$ qmodulo $\left(q_{1}, \ldots, q_{m}\right)$. Como qmodulo $\left(q_{1}, \ldots, q_{m}\right)$ é arquimedeano, o Teorema 21 nos possibilita dizer que existe $k \in \mathbb{N}$ tal que $2 k-s, 2 k-s^{2} f-1 \in$ qmodulo $\left(q_{1}, \ldots, q_{m}\right)$. Defina $h:=s(2 k-s)$ e $M:=k^{2}$. Então, $h \in \Sigma_{n} Q \subset Q$ e

$$
M-h=k^{2}-2 s k+s^{2}=(k-s)^{2} \in \Sigma_{n} .
$$

Além disso,

$$
h f-1=s(2 k-s) f-1=2 k(s f-1)+\left(2 k-s^{2} f-1\right) \in \text { qmodulo }\left(q_{1}, \ldots, q_{m}\right),
$$

pois tanto $s f-1$ quanto $2 k-s^{2} f-1$ pertencem a qmodulo $\left(q_{1}, \ldots, q_{m}\right)$. Portanto $h f \in$ $1+$ qmodulo $\left(q_{1}, \ldots, q_{m}\right)$.

Demonstremos o Teorema 22. Sob suas hipóteses, vale a tese do Lema 3. Logo, existem $h \in$ qmodulo $\left(q_{1}, \ldots, q_{m}\right)$ e $M \in \mathbb{N}$ tais que $M-h \in \Sigma_{n}$ e $h f \in 1+$ qmodulo $\left(q_{1}, \ldots, q_{m}\right)$. Além disso, existe $k \in \mathbb{N}$ tal que $k+f \in$ qmodulo $\left(q_{1}, \ldots, q_{m}\right)$, pelo Teorema 21. Então, temos que

$$
\left(k-\frac{1}{M}\right)+f=\frac{1}{M}((M-h)(k+f)+(h f-1)+k h) \in \text { qmodulo }\left(q_{1}, \ldots, q_{m}\right) .
$$

O mesmo pode ser dito sobre $(k-2 / M)+f=((k-1 / M)-1 / M)+f$, apenas substituindo $k$ no argumento acima por $k-1 / M$, pois vale que $(k-1 / M)+f \in$ qmodulo $\left(q_{1}, \ldots, q_{m}\right)$; repetindo este raciocínio com $k \leftarrow k-2 / M$, obtemos $(k-3 / M)+f \in$ qmodulo $\left(q_{1}, \ldots, q_{m}\right)$ e, com $k N$ repetições, concluímos que $f \in$ qmodulo $\left(q_{1}, \ldots, q_{m}\right)$, quod erat demonstrandum.

É importante notar, na demonstração do Teorema 22, que o resultado é válido também quando $\mathcal{K}$ é vazio. O seguinte corolário do P-satz de Putinar firma o critério de inviabilidade associado a ele.

Corolário 23. Dados $g_{1}, \ldots, g_{m} \in \mathbb{R}[\boldsymbol{x}]$, defina $\mathcal{K}:=\left\{x \in \mathbb{R}^{n} \mid g_{i} \geqslant 0, \forall i \in\{1, \ldots, m\}\right\}$ e suponha que $\mathcal{K}$ é compacto, isto é, que existe $M>0$ tal que $\mathcal{K} \subseteq\left\{x \in \mathbb{R}^{n} \mid g(x):=\right.$ $\left.M-\|x\|_{2}^{2} \geqslant 0\right\}$. Então $\mathcal{K}=\emptyset$ se, e somente se, $-1 \in$ qmodulo $\left(g, g_{1}, \ldots, g_{m}\right)$. 
Demonstração. Se existem $q_{0}, \ldots, q_{m}, q \in \Sigma_{n}$ tais que $q_{0}+g_{1} q_{1}+\cdots+g_{m} q_{m}+g q=-1$, então $\mathcal{K}=\emptyset$, pois se houvesse algum elemento $x \in \mathcal{K}$, então teríamos $q_{0}(x)+g_{1}(x) q_{1}(x)+$ $\cdots+g_{m}(x) q_{m}(x)+g(x) q(x)<0$, o que é um absurdo. Por outro lado, se $\mathcal{K}$ é vazio, então o polinômio $p: x \mapsto p(x)=-1$ é positivo em $\mathcal{K}$, por vacuidade, logo o positivstellensatz de Putinar pode ser aplicado, já que discussão anterior implica em qmodulo $\left(g, g_{1}, \ldots, g_{m}\right)$ ser aquimediano. Portanto, $-1 \in$ qmodulo $\left(g, g_{1}, \ldots, g_{m}\right)$. Além disso, se qmodulo $\left(g_{1}, \ldots, g_{m}\right)$ é arquimediano, então $\mathcal{K}=\emptyset$ se, e somente se, $-1 \in$ qmodulo $\left(g_{1}, \ldots, g_{m}\right)$.

O Corolário 23 pode ser complementado pelo procedimento descrito na Seção 3.2.4 que, por sua vez, necessita da técnica apresentada no capítulo seguinte para formar a ponte entre a geometria algébrica real e o problema de cobertura. Na prática, como polinômio $g$ depende exclusivamente de $V$, que está fixo, assumimos que qmodulo $\left(g_{1}, \ldots, g_{m}\right)$ é arquimediano.

Como existem diversas aplicações para os teoremas da classe positivstellensatz e a variável inteira $d$ representa um grande empecilho computacional, então a discussão sobre o grau dos coeficientes SOS de cada $P$-satz esteve em alta até meados de 2008. Exibimos a seguir o mais recente resultado, de J. Nie e M. Schweighofer:

Teorema 24 (P-satz de Putinar limitado). Sejam $g_{1}, \ldots, g_{m} \in \mathbb{R}[\boldsymbol{x}]$ tais que

$$
\emptyset \neq \operatorname{nonneg}\left(g_{1}, \ldots, g_{m}\right) \subseteq(-1,1)^{n} .
$$

Então, existe $c \in \mathbb{R}_{++}$(que depende dos $g_{i}$ ) tal que, para todo polinômio $f$ de grau $d$ satisfazendo

$$
f^{\star}:=\min \left\{f(x) \mid x \in \operatorname{nonneg}\left(g_{1}, \ldots, g_{m}\right)\right\}>0,
$$

vale

$$
f \in \operatorname{qmodbound}\left(g_{1}, \ldots, g_{m}, c \exp \left(d^{2} n^{d} \frac{\|f\|_{2}}{f^{\star}}\right)^{c}\right)
$$

onde

$$
\text { qmodbound }\left(g_{1}, \ldots, g_{m}, \sigma\right):=\left\{s_{0}+\sum_{i=1}^{m} s_{i} g_{i} \mid s_{i} \in \Sigma_{n, \sigma}\right\} \text {. }
$$

Há também uma versão limitada do P-satz de Schmüdgen. Mais detalhes acerca do Teorema 24 podem ser encontrados em [NS08], juntamente a diversos outros resultados sobre o grau. Um detalhe importante a se notar aqui é a hipótese nonneg $\left(g_{1}, \ldots, g_{m}\right) \neq \emptyset$, o que não nos permite restringir o Corolário 23 para um grau limitado diretamente. Contudo, como este grau, na prática, é muito menor que o limtante quando ele existe, é esperado que isso não gere complicações adicionais em âmbito computacional. 



\section{Capítulo 4}

\section{Programação semidefinida e restauração inexata}

Este capítulo tem caráter introdutório e expõe superficialmente os fundamentos de programação semidefinida, alguns algoritmos e pacotes computacionais, e um esboço da teoria geral de restauração inexata para problemas de otimização não linear.

\subsection{Programação semidefinida}

Programação (ou otimização) semidefinida (SDP) é uma área da otimização convexa, extensivamente estudada nas últimas décadas, que pode ser entendida como uma generalização da programação linear, no seguinte sentido: em vez de minimizarmos funções lineares sobre poliedros, o fazemos sobre espectraedros, que são interseções de subespaços afins e o cone das matrizes semidefinidas positivas.

\subsubsection{Caracterização}

A forma primal de um SDP linear é:

$$
\begin{array}{r}
\min C \cdot X \\
\text { sujeito a } A_{j} \cdot X=b_{j}, \forall j \in\{1, \ldots, m\} \\
X \succeq 0
\end{array}
$$

onde $C, A_{1}, \ldots, A_{m} \in \mathcal{S}^{n}, b:=\left(b_{1}, \ldots, b_{m}\right) \in \mathbb{R}^{m}$ e $X \in \mathbb{R}^{n \times n}$ é a variável de decisão. Quando lidamos com problemas que se apresentam de modo que possuem mais de uma variável, por exemplo,

$$
\begin{array}{r}
\min C_{1} \cdot X_{1}+C_{2} \cdot X_{2} \\
\text { sujeito a } A_{j 1} \cdot X_{1}+A_{j 2} \cdot X_{2}=b_{j}, \forall j \in\{1, \ldots, m\} \\
X_{1} \succeq 0 \\
X_{2} \succeq 0
\end{array}
$$

é possível reescrevê-lo na forma (4.1) com uma simples mudança de variável envolvendo diagonais em blocos. Para constar: 


$$
X:=\left[\begin{array}{cc}
X_{1} & 0 \\
0 & X_{2}
\end{array}\right] \quad C:=\left[\begin{array}{cc}
C_{1} & 0 \\
0 & C_{2}
\end{array}\right] \quad A_{j}:=\left[\begin{array}{cc}
A_{j 1} & 0 \\
0 & A_{j 2}
\end{array}\right], \forall j \in\{1, \ldots, m\} .
$$

O mesmo procedimento pode ser aplicado para um número arbitrário de variáveis. Além disso, se $X_{k}=\left[x_{i j}^{k}\right]_{i, j \in\{1, \ldots, n\}}$, sendo $k \in \mathbb{N}$ fixo, desigualdades ou igualdades lineares envolvendo apenas alguns de seus elementos, como $x_{21}^{1}+3 x_{22}^{1}+2 x_{11}^{2}=0$ (com $n=k=2$, por exemplo), podem ser reescritas na forma (4.1) utilizando coeficientes matriciais com as entradas correspondentes não nulas e as demais nulas. No caso exibido, tomar

$$
A_{1}:=\left[\begin{array}{cc}
0 & 1 / 2 \\
1 / 2 & 3
\end{array}\right] \quad A_{2}:=\left[\begin{array}{ll}
2 & 0 \\
0 & 0
\end{array}\right]
$$

nos levaria é equação equivalente $A_{1} \cdot X_{1}+A_{2} \cdot X_{2}=0$. Da mesma forma, podemse considerar variáveis matriciais de tamanhos diferentes, desde que sejam quadradas e simétricas. Portanto, é suficientemente abrangente atentarmos apenas ao Problema 4.1.

Espectraedros são conjuntos semialgébricos, convexos, fechados e, portanto, suas projeções também são semialgébricas (Tarski-Seidenberg) e fechadas. Porém, o conjunto de soluções de um SDP não é poliédrico, como as do LP e, mesmo que os dados sejam racionais, as soluções não serão necessariamente racionais [PBT13]. Quando a função objetivo do SDP é zero, (4.1) é dito ser um problema de viabilidade.

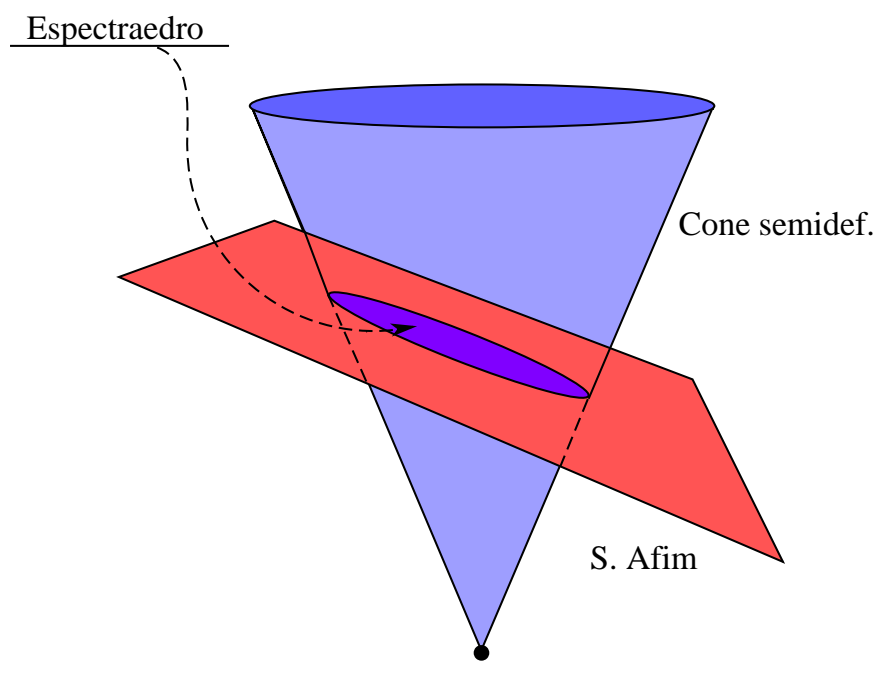

Figura 4.1: Representação de um espectraedro, como interseção de $\mathcal{S}_{+}^{n}$ com um subespaço afim.

A seguir, veremos um pequeno exemplo de como representar um problema na forma de SDP.

Exemplo 8 (Menor valor próprio). Seja $A \in \mathcal{S}^{n}$ e suponha que $\lambda_{1} \geqslant \cdots \geqslant \lambda_{n}$ são seus autovalores ordenados; para calcular $\lambda_{n}$, note que os autovalores de $A-t I$ são $\lambda_{1}-t, \ldots, \lambda_{n}-t$ (pois $A v=\lambda v$ se, e somente se, $(A-t I) v=(\lambda-t) v$, sendo $v$ um autovetor de $A$ associado ao autovalor $\lambda$ ) e que estes são não negativos quando $t \leqslant \lambda_{n}$ ou, equivalentemente, quando $A-t I \succeq 0$; sendo assim o menor autovalor de $A$ é obtido quando $t=\lambda_{n}$ e este valor é, de fato, atingido pois $\lambda_{i}-\lambda_{n} \geqslant 0$ para todo $i \in\{1, \ldots, n\}$. Logo, $A-\lambda_{n} I \succeq 0$, de modo que o menor autovalor de A é exatamente a solução do problema 


$$
\begin{array}{r}
\max t \\
\text { sujeito a } t I-A \preceq 0
\end{array}
$$

Alternativamente, defina $\mathcal{A}:=A-\lambda_{n} I \succeq 0$ e, para qualquer matriz semidefinida positiva $X$ tal que $X \cdot I=\operatorname{tr}(X)=1$, vale

$$
\mathcal{A} \cdot X=\operatorname{tr}\left(\mathcal{A}^{t} X\right)=\operatorname{tr}(\mathcal{A} \sqrt{X} \sqrt{X})=\operatorname{tr}(\sqrt{X} \mathcal{A} \sqrt{X}) \geqslant 0
$$

pois $\sqrt{X} \mathcal{A} \sqrt{X} \succeq 0$ (isso segue do Teorema 1). Logo, $\mathcal{A} \cdot X=A \cdot X-\lambda_{n} X \cdot I \geqslant 0$ e, consequentemente, $A \cdot X \geqslant \lambda_{n} X \cdot I=\lambda_{n}$. Portanto $\lambda_{n}$ também é solução do problema

$\min A \cdot X$

sujeito a $X \cdot I=1$

$X \succeq 0$

isto é, obtivemos uma representação deste problema na forma (4.1) e, anteriormente, uma outra da mesma natureza, mas com um formato aparentemente distinto.

A relação entre essas duas formulações será explicada na subseção seguinte.

\subsubsection{Funções Lagrangiana e dual}

Similarmente ao LP, existe uma forma dual associada a qualquer SDP primal obtida a partir da penalização do Lagrangiano do problema (4.1).

Definição 12 (Lagrangiano). A função Lagrangiana do problema (4.1) é dada por

$$
\begin{aligned}
\mathcal{L}(X, s, y) & :=C \cdot X+\sum_{j=1}^{m} y_{j}\left(b_{j}-A_{j} \cdot X\right)-s \lambda_{\min }(X) \\
& =y^{t} b+\left(C-\sum_{j=1}^{m} y_{j} A_{j}\right) \cdot X-s \lambda_{\min }(X),
\end{aligned}
$$

onde $\lambda_{\min }(X)$ é o menor autovalor de $X, y \in \mathbb{R}^{m}$ e $s \geqslant 0$.

Pode-se interpretar o Lagrangiano como uma espécie de penalização do problema (4.1), visto como um problema geral de otimização, uma vez que a restrição $X \succeq 0$ é equivalente a $\lambda_{\min }(X) \geqslant 0$.

Definição 13 (Funções dual e subdual). A função subdual ao Lagrangiano é definida por

$$
G(s, Z):=\min _{X} Z \cdot X-s \lambda_{\min }(X)
$$

com $Z \in \mathbb{R}^{n \times n}$. Dado isso, definimos a função dual ao Lagrangiano por

$$
\mathcal{G}(s, y):=\min _{X} \mathcal{L}(X, s, y)=y^{t} b+G\left(s, C-\sum_{j=1}^{m} y_{j} A_{j}\right) .
$$

Um $\operatorname{par}(s, y)$ tal que $\mathcal{G}(s, y)>-\infty$ é dito ser viável dual.

A função dual representa a solução do problema penalizado irrestrito de minimizar o Lagrangiano para parâmetros $s$ e $y$ fixos. De acordo com a teoria geral, o valor da função dual converge para uma solução do problema (4.1) conforme os parâmetros tendem ao infinito em valor absoluto. 
Como $\lambda_{\min }(X)=\min \left\{Y \cdot X \mid Y \in \mathcal{S}_{+}^{n}, \operatorname{tr}(Y)=1\right\}$ (Exemplo 8), temos que

$$
\begin{gathered}
G(s, Z)=\min _{X}\left(Z \cdot X-\min _{Y \succeq 0, \operatorname{tr}(Y)=1} s Y \cdot X\right) \\
=\min _{X} \min _{Y \succeq 0, \operatorname{tr}(Y)=1}(Z-s Y) \cdot X .
\end{gathered}
$$

Substituindo $Y$ por $s Y$ e reescrevendo, temos

$$
\begin{gathered}
G(s, Z)=\min _{X} \min _{Y \succeq 0, \operatorname{tr}(Y)=s}(Z-Y) \cdot X \\
=\min _{Y \succeq 0, \operatorname{tr}(Y)=s} \min _{X}(Z-Y) \cdot X .
\end{gathered}
$$

Mas $\min _{X}(Z-Y) \cdot X$ é igual a zero quando $Z=Y$ e $-\infty$, caso contrário.

Isso implica que $G(s, Z)=0$, se $\operatorname{tr}(Z)=s \geqslant 0$ e $Z \succeq 0$, mas $G(s, Z)=-\infty$, caso contrário. Note que $Z \succeq 0$ implica em $\operatorname{tr}(Z) \geqslant 0$. Logo, essa restrição é dispensável, e o mesmo vale para a função dual $\mathcal{G}$. Por isso, concluímos que os pontos viáveis duais são os $s$ e $y$ tais que

$$
C \succeq \sum_{j=1}^{m} y_{j} A_{j}
$$

afirmação que pode ser ratificada avaliando o subdual em $Z=C-\sum_{j=1}^{m} y_{j} A_{j}$.

Note que para todo $X$ viável de (4.1) e $s \geqslant 0$, temos

$$
\mathcal{L}(X, s, y)=C \cdot X-s \lambda_{\min }(X) \leqslant C \cdot X
$$

Logo, $\mathcal{G}(s, y) \leqslant p^{\star}$, se $p^{\star}$ é solução de (4.1).

Acabamos de formular intuitivamente o problema dual de 4.1, pois maximizar a função dual sobre os pontos viáveis duais nos fornece um ótimo limitante inferior para as soluções do problema primal, segundo esse ponto de vista. Formalmente, o dual de um SDP é dado por:

$$
\begin{array}{r}
\max b^{t} y \\
\text { sujeito a } \sum_{j=1}^{m} A_{j} y_{j} \preceq C
\end{array}
$$

onde $b=\left(b_{1}, \ldots, b_{m}\right)$ é o custo e $y=\left(y_{1}, \ldots, y_{m}\right)$ é a nova variável de decisão. A restrição de (4.3) é dita ser uma desigualdade linear de matrizes (LMI) e pode, é claro, ser substituída pela restrição $\sum_{j=1}^{m} A_{j} y_{j}+S=C$, juntamente com $S \succeq 0$. Além disso, mudanças de variáveis análogas às discutidas anteriormente nos permitem reescrever um sistema de LMIs como apenas um.

Um fato mencionável é que a dualidade é simétrica, no sentido que o dual de (4.3) é (4.1).

Outro fato interessante é que é possível definir uma versão cônica do Lagrangiano para o problema (4.1) como

$$
\mathcal{L}^{\star}(X, S, y):=C \cdot X+\sum_{j=1}^{m} y_{i}\left(b_{i}-A_{j} \cdot X\right)-S \cdot X
$$

e deduzir o problema dual de maneira análoga à anterior, mas trazendo uma vantagem em termos de notação; maiores detalhes em [Gha08]. 


\subsubsection{Dualidade}

Assim como no caso linear, está presente o conceito de dualidade fraca, que já esboçamos ao deduzir o problema dual e agora reescreveremos formalmente segundo os formatos fixados em (4.1) e (4.3).

Proposição 25 (Dualidade fraca). Sejam X um ponto viável de (4.1) e y um ponto viável do problema dual (4.3). Então $C \cdot X \geqslant b^{t} y$ e, portanto $d^{\star} \leqslant p^{\star}$, sendo que $d^{\star}$ denota um valor ótimo de (4.3) e $p^{\star}$, um de (4.1).

Demonstração. Se $y$ é viável no dual, então $\sum_{j=1}^{m} A_{j} y_{j}+S=C$, mas o fato de $X$ ser viável para o primal implica em

$$
C \cdot X=\sum_{j=1}^{m}\left(A_{j} \cdot X\right) y_{j}+S \cdot X=b^{t} y+S \cdot X \geqslant b^{t} y,
$$

porque $S, X \in \mathcal{S}_{+}^{n}$ e o item 3 da Proposição 2 nos permite concluir que $S \cdot X \geqslant 0$. A última afirmação é direta.

Contudo, de maneira geral não está presente o conceito de dualidade forte, como havia em LP, mas existe o seguinte resultado, devido a Morton Slater:

Teorema 26 (Dualidade forte). Considere os problemas primal e dual com valores ótimos $p^{\star}$ e $d^{\star}$, respectivamente. Se (4.1) (respec. (4.3)) é limitado inferiormente (respec. superiormente) e estritamente viável, isto é, se existe $X \succ 0$ tal que $A_{j} \cdot X=b_{j}$ para todo $j \in\{1, \ldots, m\}$ (análogo para o dual), então $p^{\star}=d^{\star}$ e a solução do dual (respec. primal) é atingida.

Uma demonstração elementar pode ser encontrada em [Tan15]. O exemplo a seguir ilustra um caso onde não é satisfeita a hipótese do Teorema 26 e não há dualidade forte.

Exemplo 9. Considere o seguinte SDP na forma (4.3):

$$
\begin{array}{r}
\max -y_{2} \\
\text { sujeito a } y_{1} A_{1}+y_{2} A_{2} \preceq A_{0}
\end{array}
$$

onde

$$
A_{0}:=\left[\begin{array}{ccc}
1 & 0 & 0 \\
0 & 0 & 0 \\
0 & 0 & 0
\end{array}\right] \quad A_{1}:=\left[\begin{array}{ccc}
0 & 0 & 0 \\
0 & -1 & 0 \\
0 & 0 & 0
\end{array}\right] \quad A_{2}:=\left[\begin{array}{ccc}
-1 & 0 & 0 \\
0 & 0 & -1 \\
0 & -1 & 0
\end{array}\right]
$$

Os determinantes dos menores de $-A_{0}+y_{1} A_{1}+y_{2} A_{2}$ são $d_{1}:=-\left(y_{2}-1\right), d_{2}:=-y_{1}$, $d_{3}:=0, d_{4}:=y_{1}\left(y_{2}-1\right), d_{5}:=-y_{2}^{2}$ e $d_{6}:=y_{2}^{2}\left(y_{2}-1\right)$. Logo, todo ponto viável dual $y$ (onde $d_{i} \leqslant 0$ ) satisfaz $y_{2}=0$, pois $d_{6} \geqslant 0$ e $y_{2}-1 \geqslant 0$. Assim, a solução d deste problema é zero.

O dual deste problema pode ser calculado mecanicamente:

$$
\begin{array}{r}
\min A_{0} \cdot Y=Y_{11} \\
\text { sujeito a } A_{1} \cdot Y=-Y_{22}=0 \\
A_{2} \cdot Y=-Y_{11}-2 Y_{23}=-1 \\
Y \succeq 0 .
\end{array}
$$


Agora, qualquer ponto viável deste problema possui o determinante do menor $2 \times 2$ inferior direita de $Y, \bar{d}:=-Y_{23}^{2}$ (pois $Y_{22}=0$ ), não negativa. Logo, $Y_{23}=0$ e $Y_{11}=1$ em todo ponto viável. Portanto a solução $p^{\star}$ deste problema é 1 , que é diferente de $d^{\star}$.

Podemos verificar que a hipótese do Teorema 26 não é satisfeita, por exemplo, notando que o determinante do menor principal $2 \times 2$ de $Y$ é $-Y_{12}^{2}$, que deve ser zero quando $Y$ é viável. Logo, não existe $Y$ definida positiva e factível.

Agora que a questão de dualidade foi formalizada, é interessante revisitar o Exemplo 8 e verificar que, de fato, as duas representações do problema do menor autovalor possuem uma relação de dualidade, mesmo tendo sido obtidas de maneiras completamente distintas.

\subsubsection{Pontos interiores}

A primeira observação a ser feita sobre algoritmos para resolução de SDPs é que não existe um análogo direto de "solução básica"do Método Simplex de LP para SDPs, isto é, o algoritmo mais popular de programação linear ainda não pode ser utilizado para resolver SDPs. Logo, as semelhanças entre essas duas classes de problemas acabam aqui. Porém, existem algoritmos baseados no esquema de pontos interiores, que serão abordados nesta seção. Antes disso, expomos condições de otimalidade para SDPs lineares:

Teorema 27 (Condições de Karush-Kuhn-Tucker (KKT)). Considere os problemas (4.1) $e$ (4.3) com valores ótimos $p^{\star} e d^{\star}$. Se ambos atingem seus valores ótimos e são estritamente viáveis, isto é, $p^{\star}=d^{\star}$, então um par primal-dual $(X, y)$ é ótimo se, e somente se, as seguintes condições são satisfeitas:

1. $\sum_{j=1}^{m} A_{j} y_{j} \preceq C$ (viabilidade dual);

2. $A_{j} \cdot X=b_{j}, \forall j \in\{1, \ldots, m\}$ e $X \succeq 0$ (viabilidade primal);

3. $X \cdot\left(\sum_{j=1}^{m} A_{j} y_{j}-C\right)=0$ (folgas complementares).

Note que os itens do Teorema 27 são análogos às condições KKT para LPs, que a viabilidade dual surge da condição $\nabla_{X} \mathcal{L}(X, S, y)=0$, com $S \succeq 0$, e que as folgas complementares surgem da dualidade forte. Uma discussão mais detalhada sobre o chamado Lagrangiano cônico descrito anteriormente pode ser encontrada em [Gha08].

Faremos, a seguir, uma breve menção aos algoritmos de pontos interiores para SDPs. Normalmente, eles são baseados em algoritmos de barreira e penalidade. Em particular, focaremos na função de barreira logarítmica, cujo argumento é uma matriz $X$ com autovalores $\lambda_{i}(X), i \in\{1, \ldots, n\}$,

$$
B(X):=-\sum_{i=1}^{n} \ln \left(\lambda_{i}(X)\right)=-\ln \left(\prod_{i=1}^{n} \lambda_{i}(X)\right)=-\ln (\operatorname{det}(X))
$$

para penalizar a fronteira de $\mathcal{S}_{+}^{n}$, isto é, consideremos o problema a seguir:

$$
\begin{array}{r}
\max C \cdot X+\mu B(X) \\
\text { sujeito a } A_{j} \cdot X=b_{j}, \forall j \in\{1, \ldots, m\} \\
X \succ 0,
\end{array}
$$


com $\mu>0$. O problema (4.4) é chamado SDP com barreira logarítmica e será denotado por $B S D P(\mu)$. O gradiente da função $f_{\mu}(X):=C \cdot X-\mu \ln (\operatorname{det}(X))$ é

$$
\nabla f_{\mu}(X)=C-\mu X^{-1}
$$

que está bem definido quando $X \succ 0$. Fazendo uma mudança de variável $S=\mu X^{-1}$, temos $X S=S X=\mu I$. Escrevendo as condições KKT deste problema,

- $A_{j} \cdot X=b_{j}, \forall j \in\{1, \ldots, m\}$ e $X \succ 0$;

- $\sum_{j=1}^{m} y_{j} A_{j}+S=C$;

- $X S=\mu I$,

é possível verificar que qualquer solução $(X, S, y)$ deste sistema tem $X$ viável primal e $(S, y)$ viável dual, com gap de dualidade

$$
C \cdot X-b^{t} y=S \cdot X=\operatorname{tr}(S X)=\operatorname{tr}(\mu I)=n \mu,
$$

sendo que a primeira igualdade é proveniente da demonstração da Proposição 25.

Tendo em mente que (4.4) sempre é estritamente viável quando (4.1) é viável, tudo o que foi discutido sugere que, quando $\mu$ tende a zero, as soluções de $B S D P(\mu)$ convergem para uma solução de (4.1). Finalmente, para resolver os subproblemas $B S D P(\mu)$ pode-se utilizar o Método de Newton nos sistemas KKT correspondentes.

\subsubsection{Solvers computacionais}

Encerramos a seção de programação semidefinida citando o solver SDPT3 (versão 4.0) de Kim-Chuan Toh, Michael J. Todd e Reha H. Tutuncu e o solver SeDuMi (versão 1.05 R5) de Jos F. Sturm, Oleksandr Romanko e Imre Pólik, que foram implementados em MATLAB e utilizam pontos interiores para resolver SDPs lineares, mas com focos diferentes: o SDPT3 aborda estes problemas como casos particulares de SDPs quadráticas e o SeDuMi abrange toda a classe de problemas de minimização sobre cones autoduais, no sentido de análise convexa, por exemplo, $\mathbb{R}_{++}^{n}$ e $\mathcal{S}_{+}^{n}$. Maiores detalhes podem ser encontrados em [Stu99] e em [TTT99].

Normalmente, a geração de entradas para os solvers é um processo que consome muito tempo. Logo, é comum a utilização de geradores de entrada para eles. Após a criação de um gerador de entradas próprio voltado ao problema de cobertura, nós nos deparamos com uma enorme dificuldade para lidar com o alto consumo de memória por parte das matrizes esparsas sem otimização de código. Isso culminava numa execução lenta de um algoritmo (a ser introduzido no capítulo seguinte) que já é intrinsecamente custoso. Sendo assim, optamos por utilizar o YALMIP (versão disponibilizada em agosto de 2017) de Johan Löfberg (veja [Lö09]) e obtivemos um resultado mais promissor ${ }^{1}$.

\footnotetext{
${ }^{1}$ Sugestão de J. Gouveia (Universidade de Coimbra) durante a SIAM Conference in Applied Algebraic Geometry, 2017.
} 


\subsection{Restauração Inexata}

Iniciaremos esta seção com uma breve motivação construtiva por trás dos chamados métodos de restauração inexata para, então, introduzi-los com mais detalhes num nível suficiente de formalidade.

A abordagem mais intuitiva para a resolução de problemas do tipo

$$
\begin{array}{r}
\min f(x) \\
\text { sujeito a } x \in \mathcal{K},
\end{array}
$$

é calcular repetitivamente, para todo ponto viável $x$ não ótimo, uma direção $d$ que mantenha a viabilidade, isto é, encontrar $d$ tal que $x+\alpha d \in \mathcal{K}$ para algum $\alpha>0$ e, ao mesmo tempo, que decresça o valor da função objetivo, ou seja, que $f(x+\alpha d)<f(x)$. Gerar uma sequência deste tipo caracteriza os chamados métodos viáveis e funciona bem quando $f$ pode ser exatamente avaliada (desconsiderando o erro intrínseco da representação em ponto flutuante) e $\mathcal{K}$ permite que essa direção $d$ sempre exista. Porém, estas são hipóteses raramente satisfeitas. Por exemplo, existem muitos casos em que $\mathcal{K}$ é determinado por equações não lineares e não vale a segunda. Por outro lado, sempre que $\mathcal{K}$ é uma interseção de conjuntos descritos por transformações lineares, é possível aplicar um método viável.

Em geral, a perda eventual de viabilidade é esperada e os algoritmos que tentarem resolver (4.5) devem ser capazes de retornar ao conjunto viável a partir de um ponto qualquer, o que caracteriza um processo chamado de restauração e dá origem a uma grande classe de métodos, como o gradiente reduzido generalizado (GRG) de J. Abadie e J. Carpentier (em [AC68]) e o método de projeção do gradiente de J. B. Rosen (veja [Ros60] e [Ros61]). Para ser perfeitamente aplicada, a restauração exige que seja possível retornar a $\mathcal{K}$ exatamente com custo computacional aceitável, por exemplo, por meio de uma projeção. Todavia, os métodos de projeção em conjuntos não linearmente representáveis são, em geral, iterativos, o que faz com que toda restauração desse tipo tenha uma medida de erro associada, tornando-as tecnicamente inexatas.

Existem ainda casos que ferem a primeira hipótese, isto é, nos quais não é possível avaliar o valor de $f(x)$ ou de suas derivadas exatamente, mas apenas associado a uma medida de erro. Por exemplo:

- Quando os valores de $f$ são dados por integrais definidas sem soluções analíticas conhecidas e, portanto, têm que ser aproximadas computacionalmente (por exemplo, em problemas de controle [BBM15]);

- Quando $f$ envolve o cálculo das médias sobre conjuntos $\Omega$ inaceitavelmente grandes, mas é aproximada por médias sobre amostras $A \subset \Omega$ de tamanhos aceitáveis; isso gera um erro em função de $A$.

Nestes casos, menor passo de integração e uma amostra maior são meios de diminuir os erros na avaliação de $f$, respectivamente. Então, introduzir uma nova restrição ao problema original que force o erro a ser nulo (caso ele possa ser escrito de maneira amigável) permite que tiremos vantagem dos meios acima descritos tratando-os como métodos de restauração. Veja [BKM17] ou [BKM18] para um desenvolvimento mais aprofundado, pois ao longo do texto desconsideraremos esse caso. 


\subsubsection{Uma visão geral}

A discussão anterior aponta uma certa peculiaridade com restrições não lineares de igualdade, o que nos motiva a discriminá-las reescrevendo (4.5) na forma:

$$
\begin{array}{r}
\min f(x) \\
\text { sujeito a } h(x)=0 \\
x \in \mathcal{D},
\end{array}
$$

onde $f: \mathbb{R}^{n} \rightarrow \mathbb{R}, h: \mathbb{R}^{n} \rightarrow \mathbb{R}^{m}$ e $\mathcal{D} \subseteq \mathbb{R}^{n}$ é fechado e convexo. Assumiremos também que $\nabla f$ e $J_{h}(x)$ existem e são contínuas em $\mathcal{D}$.

A restauração inexata (RI) é um paradigma computacional para resolução de problemas do tipo (4.6) baseando-se nas ideias de restauração, mas tratando viabilidade e otimalidade separadamente. Esta classe de métodos toma direções na aproximação linear do conjunto factível, de modo a decrescer a função objetivo, tolerando eventuais perdas razoáveis de viabilidade e, na etapa seguinte, recupera o suficiente dela, de modo a não perder muito do decréscimo recém obtido. Inclusive, o meio de restauração pode ser escolhido arbitrariamente, de modo a aproveitar as particularidades de cada problema. Essa é uma das justificativas para a utilização de um método de duas fases, que pode parecer desvantajoso à primeira vista.

\subsubsection{A fase de restauração}

Dado um ponto $x \in \mathcal{D}$, defina $\bar{x} \in \mathcal{D}$ como sua contraparte restaurada. O mínimo exigido de uma restauração é que $\bar{x}$ seja "mais viável" que $x$ com respeito a $h$, isto é, que

$$
\|h(\bar{x})\|_{2} \leqslant \gamma\|h(x)\|_{2}
$$

para algum $\gamma \in(0,1)$. Se isso não for possível, então o restante do algoritmo também pode falhar (veja mais adiante, no Teorema 28).

\subsubsection{A fase de minimização}

Sabendo que num conjunto linear convexo sempre é possível obter uma direção viável e de decréscimo para $f$, buscamos uma direção $d$ que mantenha $x+d$ quase tão viável quanto $x$ relativo a $h$, ou seja, tal que

$$
h(x+d) \approx h(x)+J_{h}(x) d=h(x) \Rightarrow J_{h}(x) d=0 .
$$

Além disso, é preciso que $x+d \in \mathcal{D}$ e que $f(x+d) \leqslant f(x)$, isto é, $d$ deve ser solução do problema

$$
\begin{array}{r}
\min _{d} f(x+d) \\
\text { sujeito a } J_{h}(x) d=0 \\
d \in \mathcal{D}-x .
\end{array}
$$

Note que (4.7) é viável, pois $0 \in \operatorname{Ker}\left(J_{h}(x)\right) \cap(\mathcal{D}-x)$, já que $x \in \mathcal{D}$.

Obtida uma boa direção, considere a seguinte função de mérito: 


$$
\Phi(x, \theta):=\theta f(x)+(1-\theta)\|h(x)\|_{2}, \operatorname{com} \theta \in[0,1],
$$

que nada mais é que uma média ponderada entre o valor de $f$ e a medida de inviabilidade $\|h(x)\|_{2}$, isto é, $\theta$ é a importância do valor da função objetivo e $(1-\theta)$, da viabilidade, tornando as duas coisas entrelaçadas, de modo que decrescer a função de mérito implica em melhorar ambas as coisas. Contudo, apenas decrescê-la não garante bons resultados, mas é necessário que ela decresça o suficiente. Para isso, exigiremos que o decréscimo real de $\Phi$,

$$
\begin{gathered}
D R_{\Phi}(\alpha, \theta):=\Phi(x, \theta)-\Phi(\bar{x}+\alpha d, \theta) \\
=\theta(f(x)-f(\bar{x}+\alpha d))+(1-\theta)\left(\|h(x)\|_{2}-\|h(\bar{x}+\alpha d)\|_{2}\right)
\end{gathered}
$$

seja suficientemente grande. Logo, definimos também o decréscimo previsto de $\Phi$ como

$$
D P_{\Phi}(\alpha, \theta):=\theta(f(x)-f(\bar{x}+\alpha d))+(1-\theta)\left(\|h(x)\|_{2}-\|h(\bar{x})\|_{2}\right)
$$

e exigimos que

$$
D R_{\Phi}(\alpha, \theta) \geqslant(1-\gamma) D P_{\Phi}(\alpha, \theta) .
$$

Mesmo que $D P_{\Phi}(\alpha, \theta)$ possa ser negativo, quando $\theta$ é suficientemente pequeno é possível garantir que

$$
D P_{\Phi}(\alpha, \theta) \geqslant \frac{1}{2}\left(\|h(x)\|_{2}-\|h(\bar{x})\|_{2}\right) \geqslant 0,
$$

devido a (4.7) e a exigência da fase de restauração. Logo, quando a desigualdade

$$
\Phi(\bar{x}+\alpha d, \theta)-\Phi(x, \theta) \leqslant \frac{1}{2}(1-\gamma)\left(\|h(\bar{x})\|_{2}-\|h(x)\|_{2}\right)
$$

é satisfeita, o tamanho de passo $\alpha$ pode ser aceito. Caso contrário, reduz-se o valor de $\alpha$.

\subsubsection{Algoritmo formal}

O algoritmo a seguir formaliza as ideias discutidas anteriormente. A ideia original de José Martínez e Elvio Pilotta (veja [MP00]) utiliza regiões de confiança e, mais tarde, uma abordagem com busca linear foi publicada por Andreas Fischer e Ana Friedlander (veja [FF10]). Nós utilizamos uma versão mais flexível e recente, publicada por Luis F. Bueno, Gabriel Haeser e José M. Martínez (veja [BHM14] e [BHM15]).

Algoritmo 2 (Fischer-Friedlander flexível).

Dados $x_{0} \in \mathcal{D}, \theta_{-1} \in(0,1), \gamma \in(0,1)$ e $\beta>0$, inicie com $k:=0$.

1. Compute $\overline{x_{k}} \in \mathcal{D}$ tal que

$$
\left\|h\left(\overline{x_{k}}\right)\right\|_{2} \leqslant \gamma\left\|h\left(x_{k}\right)\right\|_{2}
$$

e

$$
f\left(\overline{x_{k}}\right)-f\left(x_{k}\right) \leqslant \beta\left\|h\left(x_{k}\right)\right\|_{2} ;
$$

2. Compute uma direção $d_{k} \in \mathbb{R}^{n}$ (solução de (4.7)) após substituir $x$ por $\overline{x_{k}}$. Se $d_{k}=0$, pare o algoritmo. Senão:

3. Defina $\theta_{k}$ como o maior $\theta \in\left[0, \theta_{k-1}\right]$ tal que

$$
\Phi\left(\overline{x_{k}}, \theta\right)-\Phi\left(x_{k}, \theta\right) \leqslant \frac{1}{2}(1-\gamma)\left(\left\|h\left(\overline{x_{k}}\right)\right\|_{2}-\left\|h\left(x_{k}\right)\right\|_{2}\right) ;
$$


4. Defina $\alpha_{k}$ como o maior $\alpha \in(0,1]$ tal que

$$
\Phi\left(\overline{x_{k}}+\alpha d_{k}, \theta_{k}\right)-\Phi\left(x_{k}, \theta_{k}\right) \leqslant \frac{1}{2}(1-\gamma)\left(\left\|h\left(\overline{x_{k}}\right)\right\|_{2}-\left\|h\left(x_{k}\right)\right\|_{2}\right)
$$

$e$

$$
f\left(\overline{x_{k}}+\alpha d_{k}\right)<f\left(\overline{x_{k}}\right)
$$

5. faça $x_{k+1} \leftarrow \overline{x_{k}}+\alpha_{k} d_{k}, k \leftarrow k+1$ e retorne ao passo 1 ;

Nesse algoritmo, os Passos 1 e 2 são as fases de restauração e minimização, respectivamente, enquanto os Passos 3 e 4 compõem a estratégia de globalização do método, discutida na seção anterior. Pela praticidade, $\alpha_{k}$ no Passo 4 pode ser escolhido no conjunto $\left\{2^{-\eta} \mid \eta \in \mathbb{N}\right\}$.

\subsubsection{Um comentário sobre convergência}

O conjunto viável de (4.7),

$$
T(x):=\left\{d \in \mathbb{R}^{n} \mid J_{h}(x) d=0, x+d \in \mathcal{D}\right\},
$$

como já discutido anteriormente é, além de não vazio, convexo e fechado, pois $\mathcal{D}$ possui essas características e $\operatorname{Ker}\left(J_{h}(x)\right)$ é uma interseção de hiperplanos.

Lema 4. Suponha que $h$ é continuamente diferenciável em $\mathcal{D}$ tal que $J_{h}$ é Lipschitz contínua em $\mathcal{D}$ com constante $L_{h}$ positiva. Então, para todo $x \in \mathcal{D}$, existe $\mu>0$ tal que:

$$
\left\|h\left(x+\alpha d^{\star}\right)\right\|_{2} \leqslant\|h(x)\|_{2}+\mu \alpha^{2}\left\|d^{\star}\right\|_{2}^{2},
$$

para todo $\alpha \in(0,1)$, sendo d* a solução de (4.7).

Demonstração. Como $J_{h}(x) d^{\star}=0$,

$$
h\left(x+\alpha d^{\star}\right)=h(x)+\alpha \int_{0}^{1}\left(J_{h}\left(x+\sigma \alpha d^{\star}\right)-J_{h}(x)\right)^{t} d^{\star} d \sigma .
$$

Logo,

$$
\begin{gathered}
\left\|h\left(x+\alpha d^{\star}\right)\right\|_{2} \leqslant\|h(x)\|_{2}+\alpha \int_{0}^{1}\left\|J_{h}\left(x+\sigma \alpha d^{\star}\right)-J_{h}(x)\right\|_{2}\left\|d^{\star}\right\|_{2} d \sigma \\
\leqslant \alpha L_{h}\left\|x+\alpha d^{\star}-x\right\|_{2}\left\|d^{\star}\right\|_{2}=\alpha^{2} L_{h}\left\|d^{\star}\right\|_{2}^{2} .
\end{gathered}
$$

Defina $\mu:=L_{h}$ e a demonstração está encerrada.

O Lema 4 está associado ao ganho "suficiente" de viabilidade ao longo da direção escolhida e sua hipótese não é um problema, uma vez que tratamos de polinômios; da mesma forma, assumiremos que existe ganho suficiente em otimalidade.

Hipótese 1. Suponha que $f$ é diferenciável e que existem $\nu>0$ e $1>\tau>0$ tais que

$$
f\left(x+\alpha d^{\star}\right) \leqslant f(x)-\nu \alpha\left\|d^{\star}\right\|_{2}^{2},
$$

para todo $\alpha \in(0, \tau)$, onde $d^{\star}$ é solução de (4.7). 
Essa hipótese não é tão restritiva, por exemplo, quando $d^{\star}$ é uma solução global de (4.7), pois, como $d=0$ é viável,

$$
f\left(x+\alpha d^{\star}\right)-f(x)=\alpha \nabla f(x)^{t} d^{\star}+\mathcal{O}\left(\alpha^{2}\left\|d^{\star}\right\|_{2}^{2}\right) \leqslant 0 .
$$

Então, quando $\alpha>0$ é pequeno o suficiente vale

$$
\alpha \nabla f(x)^{t} d^{\star}=\alpha\|\nabla f(x)\|_{2}\left\|d^{\star}\right\|_{2} \cos \theta \leqslant 0 .
$$

Defina $\nu:=-\|\nabla f(x)\|_{2}\left\|d^{\star}\right\|_{2}^{-1} \cos \theta$ e a hipótese será satisfeita quando $\nabla f(x) \neq 0$ e $d^{\star} \neq 0$ e quando $\cos \theta \neq 0$. No caso do problema de cobertura, como será discutido mais à frente, cada instância de (4.7) minimiza uma função linear com valor limitado inferiormente num conjunto fechado. Logo, há solução global. Além disso, $\cos \theta=0$ somente quando a direção no raio $d_{r}$ é zero, o que não é realmente um impeditivo.

Para mais detalhes, veja [FF10] ou [BHM14].

Proposição 28. Assumindo a Hipótese 1, se o Passo 1 do Algoritmo 2 está bem definido, então todos os outros passos do Algoritmo 2 também estão bem definidos, a sequência $\left\{\theta_{k}\right\}_{k \in \mathbb{N}}$ converge para um escalar maior que zero e $\left\{\alpha_{k}\right\}_{k \in \mathbb{N}}$ está limitada inferiormente por um número maior que zero.

Demonstração.

- Fixe $k \in \mathbb{N}$ e sejam $x_{k}$ e $\theta_{k}$ gerados pelo Algoritmo 2. Então, para todo $\theta \in(0,1)$,

$$
\begin{gathered}
\Phi\left(\overline{x_{k}}, \theta\right)-\Phi\left(x_{k}, \theta\right)=\theta\left(f\left(\overline{x_{k}}\right)-f\left(x_{k}\right)\right)+(1-\theta)\left(\left\|h\left(\overline{x_{k}}\right)\right\|_{2}-\left\|h\left(x_{k}\right)\right\|_{2}\right) \\
\leqslant \theta \beta\left\|h\left(x_{k}\right)\right\|_{2}-(1-\theta)(1-\gamma)\left\|h\left(x_{k}\right)\right\|_{2} \\
=\left\|h\left(x_{k}\right)\right\|_{2}(\theta(\beta+1-\gamma)-(1-\gamma)) .
\end{gathered}
$$

Agora defina

$$
\bar{\theta}:=\frac{1-\gamma}{2(\beta+1-\gamma)}
$$

e note que, se $\theta \in[0, \bar{\theta}]$ vale

$$
\theta(\beta+1-\gamma)-(1-\gamma) \leqslant-\frac{1}{2}(1-\gamma)
$$

$\mathrm{e}$

$$
\Phi\left(\overline{x_{k}}, \theta\right)-\Phi\left(x_{k}, \theta\right) \leqslant \frac{1}{2}(1-\gamma)\left(\left\|h\left(\overline{x_{k}}\right)\right\|_{2}-\left\|h\left(x_{k}\right)\right\|_{2}\right) .
$$

Logo, o Passo 3 do Algoritmo 2 está bem definido, isto é,

$$
\theta_{k}:=\max \left\{\theta \in\left[0, \theta_{k-1}\right], \text { tal que (4.8) vale }\right\}
$$

existe.

- Como qualquer $\theta \leqslant \bar{\theta} \operatorname{satisfaz~(4.8),~então,~para~todo~} k \in \mathbb{N}, \theta_{0} \geqslant \theta_{k} \geqslant \bar{\theta}$, por definição. Logo,

$$
\theta_{k} \geqslant \theta^{\star}:=\min \left\{\theta_{0}, \frac{\bar{\theta}}{2}\right\}>0
$$

pois $\bar{\theta}>0$. Daí, segue que $\left\{\theta_{k}\right\}_{k \in \mathbb{N}}$ é limitada inferiormente por $\theta^{\star}>0$ e, por definição, é monótona não crescente. Portanto, essa sequência converge e seu ponto limite é maior que zero. 
- A discussão na Subseção 4.2.3 e a Hipótese 1 nos levam concluir que o Passo 2 do Algoritmo 2 está bem definido.

- Pelo Lema 4, a Hipótese 1 e as propriedades da fase de restauração, temos que

$$
\begin{gathered}
\Phi\left(\overline{x_{k}}+\alpha_{k} d_{k}, \theta_{k}\right)-\Phi\left(x_{k}, \theta_{k}\right)=\Phi\left(\overline{x_{k}}+\alpha_{k} d_{k}, \theta_{k}\right)-\Phi\left(x_{k}, \theta_{k}\right)+\Phi\left(\overline{x_{k}}, \theta_{k}\right)-\Phi\left(\overline{x_{k}}, \theta_{k}\right) \\
\leqslant \theta_{k}\left(f\left(\overline{x_{k}}+\alpha_{k} d_{k}\right)-f\left(\overline{x_{k}}\right)\right)+\left(1-\theta_{k}\right)\left(\left\|h\left(\overline{x_{k}}+\alpha_{k} d_{k}\right)\right\|_{2}-\left\|h\left(\overline{x_{k}}\right)\right\|_{2}\right)+ \\
+\frac{1}{2}(1-\gamma)\left(\left\|h\left(\overline{x_{k}}\right)\right\|_{2}-\left\|h\left(x_{k}\right)\right\|_{2}\right) \\
<\alpha\left\|d_{k}\right\|_{2}\left(\left(1-\theta_{k}\right) \mu \alpha-\theta_{k} \nu\right)+\frac{1}{2}(1-\gamma)\left(\left\|h\left(\overline{x_{k}}\right)\right\|_{2}-\left\|h\left(x_{k}\right)\right\|_{2}\right)
\end{gathered}
$$

para algum $\mu>0, \nu>0$ e todo $\alpha \in(0, \tau)$, para algum $\tau>0$. Daí, quando $\alpha$ é suficientemente pequeno, digamos $\alpha<\alpha^{\star} \leqslant \tau$, temos

$$
\Phi\left(\overline{x_{k}}+\alpha_{k} d_{k}, \theta_{k}\right)-\Phi\left(x_{k}, \theta_{k}\right) \leqslant \frac{1}{2}(1-\gamma)\left(\left\|h\left(\overline{x_{k}}\right)\right\|_{2}-\left\|h\left(x_{k}\right)\right\|_{2}\right)
$$

e o Passo 4 também está bem definido.

- De forma análoga ao que foi discutido para $\theta_{k}$, temos que $\alpha_{k} \leqslant \alpha^{\star} / 2$ e, portanto, $\left\{\alpha_{k}\right\}_{k \in \mathbb{N}}$ está limitada inferiormente por uma constante maior que zero.

Teorema 29. Se o Passo 1 do Algoritmo 2 está bem definido e $\mathcal{D}$ é compacto, então:

1. $\lim _{k \rightarrow \infty}\left\|h\left(x_{k}\right)\right\|_{2}=\lim _{k \rightarrow \infty}\left\|h\left(\overline{x_{k}}\right)\right\|_{2}=0 ;$

2. $\lim _{k \rightarrow \infty} d_{k}=0$.

Demonstração.

1. Assumindo todas as conclusões da Proposição $28 \operatorname{com} \theta^{\star}:=\lim _{j \rightarrow \infty} \theta_{j}>0$, temos $\Phi\left(x_{k+1}, \theta^{\star}\right)-\Phi\left(x_{k}, \theta^{\star}\right) \leqslant \frac{1}{2}(1-\gamma)\left(\gamma\left\|h\left(x_{k}\right)\right\|_{2}-\left\|h\left(x_{k}\right)\right\|_{2}\right)=-\frac{1}{2}(1-\gamma)^{2}\left\|h\left(x_{k}\right)\right\|_{2}$

para todo $k \in \mathbb{N}$. Segue que, para todo $l \in \mathbb{N} \operatorname{com} l>k_{0}$,

$$
\begin{gathered}
\Phi\left(x^{l}, \theta_{k_{0}}\right)-\Phi\left(x_{k_{0}}, \theta_{k_{0}}\right)=\sum_{k=k_{0}}^{l-1}\left(\Phi\left(x_{k+1}, \theta_{k_{0}}\right)-\Phi\left(x_{k}, \theta_{k_{0}}\right)\right) \\
\leqslant-\frac{1}{2}(1-\gamma)^{2} \sum_{k=k_{0}}^{l-1}\left\|h\left(x_{k}\right)\right\|_{2} .
\end{gathered}
$$

Agora, como $\Phi$ é contínua, a compacidade de $\mathcal{D}$ implica que, para todo $l$, a soma $\sum_{k=k_{0}}^{l-1}\left\|h\left(x_{k}\right)\right\|_{2}$ é limitada e crescente em função de $l$. Logo, essa série converge e, portanto, $\lim _{k \rightarrow \infty}\left\|h\left(x_{k}\right)\right\|_{2}=0$. A conclusão sobre o limite envolvendo os pontos restaurados segue do critério de restauração. 
2. Usando as condições do Passo 1, o Lema 4 e a Hipótese 1, vale que

$$
f\left(x_{k+1}\right)-f\left(x_{k}\right)=f\left(\overline{x_{k}}+\alpha_{k} d_{k}\right)-f\left(\overline{x_{k}}\right)+f\left(\overline{x_{k}}\right)-f\left(x_{k}\right) \leqslant-\nu \alpha_{k}\left\|d_{k}\right\|_{2}^{2}+\beta\left\|h\left(x_{k}\right)\right\|_{2} .
$$

Logo,

$$
\begin{aligned}
f\left(x_{l+1}\right)-f\left(x_{0}\right)= & \left(f\left(x_{l+1}\right)-f\left(x_{l}\right)\right)+\left(f\left(x_{l}\right)-f\left(x_{l-1}\right)\right)+\cdots-f\left(x_{0}\right) \leqslant \\
& \leqslant-\nu \sum_{k=0}^{l} \alpha_{k}\left\|d_{k}\right\|_{2}+\beta \sum_{k=0}^{l}\left\|h\left(x_{k}\right)\right\|_{2},
\end{aligned}
$$

o que vale para todo $l \in \mathbb{N}$. O item 1 e o fato de que $f$ ser contínua num conjunto compacto $\mathcal{D}$ implica que $\lim _{k \rightarrow \infty} d_{k}=0$.

O Teorema 29 remete à finitude e à viabilidade do Algoritmo 2, isto é, ele garante que o algoritmo termina e que o ponto limite é viável quando $\mathcal{D}$ é compacto. Contudo, a questão de corretude envolve uma condição de qualificação chamada $C P G$, que implica que todo ponto limite gerado pelo algoritmo satisfaz as condições de otimalidade KKT, mas sem ela ao menos a condição $L$-AGP é satisfeita. Mais detalhes sobre isso em [BHM14].

Mesmo tendo fundamentadas as bases do algoritmo genérico de restauração inexata, a hipótese sobre a compacidade de $\mathcal{D}$ torna sua convergência, no mínimo, obscura para 0 caso envolvido neste trabalho, que será abordado no capítulo seguinte.

Informações mais aprofundadas acerca de restauração inexata podem ser encontradas em [MP05] e [BHM15], além das referências já citadas. 


\section{Capítulo 5}

\section{Resultados}

O último capítulo deste trabalho é um ponto de acumulação comum das duas principais sequências de raciocínio contidas nos Capítulos 3 e 4 . Iniciaremos aplicando todos os resultados algébricos exibidos anteriormente ao problema de cobertura e, em seguida, faremos algumas considerações a respeito dos algoritmos computacionais desenvolvidos, incluindo as heurísticas e comentários sobre convergência.

\section{$5.1 \quad$ Acerca do modelo}

Recordamos o problema de cobertura:

$$
\begin{array}{r}
\min r \\
\text { sujeito a } \mathcal{K}=\emptyset \\
r \geqslant 0,
\end{array}
$$

onde $\mathcal{K}:=\left\{x \in \mathbb{R}^{n} \mid q_{i}(x) \geqslant 0, p_{j}\left(x, r, c_{j}\right)>0, \forall i \in\{1, \ldots, t\}, j \in\{1, \ldots, m\}\right\}, m$ é o número de esferas, $t$ é o número de polinômios que descrevem o conjunto a ser coberto $V$ e $r$ é o raio das esferas.

Note que esse conjunto também pode ser escrito como

$$
\mathcal{K}:=\left\{x \in \mathbb{R}^{n} \mid q_{1}(x) \geqslant 0, \ldots, q_{t}(x) \geqslant 0, p_{1}\left(x, r, c_{1}\right) \geqslant 0, \ldots, p_{m}\left(x, r, c_{m}\right) \geqslant 0, \prod_{k=1}^{m} p_{k}\left(x, r, c_{k}\right) \neq 0\right\}
$$

e representa todos os pontos de $V$ que estão fora de $\mathcal{B}$. Logo, $\mathcal{K}$ é compacto quando $V$ é limitado e $r<\infty$. Para simplifcar a notação, lembramos que $C:=\left\{c_{1}, \ldots, c_{m}\right\}$ e definimos

$$
g_{i}(x, r, C):=q_{i}(x), \text { se } i \in\{1, \ldots, t\} \text { e } g_{j+t}(x, r, C):=p_{j}\left(x, r, c_{j}\right) \text {, se } j \in\{1, \ldots, m\} ;
$$

além disso,

$$
\bar{g}(x, r, C):=\prod_{k=1}^{m} p_{k}\left(x, r, c_{k}\right) .
$$

Por ser um conjunto semialgébrico básico, $\mathcal{K}$ pode ter sua eventual vacuidade certificada por polinômios que são somas de quadrados, fato decorrente do Teorema 16. Ou seja, $\mathcal{K}=\emptyset$ se, e somente se, existem $b, d \in \mathbb{N}$ e $s_{0}(x), s_{\Lambda}(x) \in \Sigma_{n, 2 d}$ para todo $\emptyset \neq \Lambda \subseteq\{1, \ldots, m+t\}$ tais que 


$$
P_{S t e}(x, r, C, s):=s_{0}(x)+\sum_{\emptyset \neq \Lambda \subseteq\{1, \ldots, m+t\}}\left(s_{\Lambda}(x) \prod_{i \in \Lambda} g_{i}(x, r, C)\right)+\bar{g}(x, r, C)^{2 b}=0,
$$

com $s:=\left(\left\{s_{0}\right\} \cup\left\{s_{\Lambda}\right\}_{\emptyset \neq \Lambda \subseteq\{1, \ldots, m+t\}}\right)$. Para constar, o grau do polinômio associado ao $P$ satz de Stengle é $\operatorname{deg}\left(P_{\text {Ste }}(x, r, C, s)\right)=2 m+2 d+\sum_{i=1}^{t} \operatorname{deg}\left(q_{i}\right)$ e, assim como foi feito no Exemplo 7, podemos escrever $s_{0}(x)=[\mathbf{x}]_{d}^{t} S_{0}[\mathbf{x}]_{d}$ e cada $s_{\Lambda}(x)=[\mathbf{x}]_{d}^{t} S_{\Lambda}[\mathbf{x}]_{d}$, para $S_{0}, S_{\Lambda} \in \mathcal{S}_{+}^{N(d)}$, lembrando que $N(d):=\left(\begin{array}{c}n+d \\ d\end{array}\right)$. Assim, para $b, d$ fixos, reduzimos (5.2) ao problema equivalente:

$$
\begin{aligned}
& \min r \\
& \text { sujeito a } \overrightarrow{P_{S t e}}(r, C, S)=\overrightarrow{0} \\
& r \geqslant 0 \text {, } \\
& S_{0} \succeq 0, \quad S_{\Lambda} \succeq 0, \forall \emptyset \neq \Lambda \subseteq\{1, \ldots, m+t\},
\end{aligned}
$$

sendo $S:=\left(\left\{S_{0}\right\} \cup\left\{S_{\Lambda}\right\} \emptyset \neq \Lambda \subseteq\{1, \ldots, m+t\}\right)$. Recordamos que $\overrightarrow{P_{S t e}}$ é o vetor de coeficientes do polinômio $P_{S t e}$ e destacamos que, quando fixamos os centros e o raio, (5.3) se torna um SDP linear de viabilidade, isto é, qualquer solução dele certifica que $\mathcal{B}(C, r)$ é uma cobertura viável para $V$. Um fato intuitivamente óbvio é que (5.1) tem solução quando $V$ é limitado.

A seguir, exibimos um exemplo ilustrando a busca de soluções de (5.2) analiticamente (algo fácil em casos pequenos) para um problema viável e mostramos a inexistência de tais soluções num caso inviável.

\section{Exemplo 10.}

- Considere dois círculos em $\mathbb{R}^{2}$, definidos pelas desigualdades $p(x, r, c):=\left(x_{1}-c_{1}\right)^{2}+$ $\left(x_{2}-c_{2}\right)^{2}-r \leq 0$ e $q(x):=-p(x, 1, \overrightarrow{0}) \geq 0$. O primeiro cobre o segundo quando o conjunto

$$
K:=\left\{x \in \mathbb{R}^{n} \mid p(x, r, c) \geq 0, q(x) \geq 0, p(x, r, c) \neq 0\right\}
$$

é vazio, o que acontece se, e somente se, existem $b, d \in \mathbb{N}$ e $s_{0}, s_{1}, s_{2}, s_{3} \in \Sigma_{n, 2 d}$ tais que

$$
P_{\text {Ste }}(x, r, c, s):=s_{0}+p s_{1}+q s_{2}+p q s_{3}+p^{2 b}=0 .
$$

Se $r=1$ e $c=\overrightarrow{0}$, ent $\tilde{a} o p=-q$ e o P-satz fica

$$
P_{S t e}(x, 1, \overrightarrow{0}, s):=s_{0}+q\left(s_{2}-s_{1}\right)-q^{2} s_{3}+q^{2 d}=0 .
$$

$\log$,

$$
s_{0}+q s_{2}+q^{2 b}=q s_{1}+q^{2} s_{3},
$$

onde um conjunto de soluções é dado por $s_{3}=q^{2(b-1)}, s_{2}=s_{1}$ arbitrários e $s_{0}=0$. Logo esta cobertura é, de fato, viável.

- Por outro lado, se c $=\overrightarrow{0}$ e $r<1$, então existe $z>0$ tal que $r=1-z$, logo $q=p-z$ $e$, se existissem $b, d \in \mathbb{N}$ e coeficientes em $\Sigma_{n, 2 d}$, tais que

$$
P_{S t e}(x, r, \overrightarrow{0}, s):=s_{0}+p s_{1}+(p-z) s_{2}+p(p-z) s_{3}+p^{2 b}=0,
$$


então tome uma sequência $\left\{x_{k}\right\}_{k \in \mathbb{N}} \rightarrow x^{\star}$, tal que $\lim _{k \rightarrow \infty}\left\|x_{k}\right\|_{2}=1$ e note que

$$
\lim _{k \rightarrow \infty} p\left(x_{k}, r, \overrightarrow{0}\right)=1-r=z,
$$

o que implica em

$$
\lim _{k \rightarrow \infty} s_{0}\left(x^{\star}\right)+z s_{1}\left(x^{\star}\right)=-z^{2 b}<0 .
$$

Mas isso implica que existe algum ponto próximo de $x^{\star}$ onde $s_{0}+z s_{1}<0$, o que é um absurdo, pois $s_{0}+z s_{1} \geqslant 0$ como polinômios. Ou seja, o círculo de centro $c=\overrightarrow{0}$ e raio $r<1$ não forma uma cobertura para $\left\{x \in \mathbb{R}^{2} \mid\|x\|_{2} \leqslant 1\right\}$, como esperado.

Mais à frente neste capítulo, comentaremos a aplicação da técnica de restauração inexata para o problema de cobertura, o que torna necessário computar a matriz jacobiana da função vetorial $\overrightarrow{P_{S t e}}(r, C, S)$. Mas, como essa é uma tarefa difícil, especialmente devido aos termos combinatoriais e exponenciais, foi utilizada a abordagem mais simples possível: aproximar cada derivada por sua definição truncada. Por exemplo,

$$
\frac{\partial \overrightarrow{P_{S t e}}(r, C, S)}{\partial r} \approx \frac{\overrightarrow{P_{S t e}}(r+\delta, C, S)-\overrightarrow{P_{S t e}}(r, C, S)}{\delta}
$$

para $0<\delta \approx 0$

\subsubsection{Cobrindo com bolas abertas}

Como o polinômio $\bar{g}$ representa os pontos de $V$ cobertos pela fronteira das esferas, removê-lo constitui um problema de cobrir um objeto fechado com uma união de bolas abertas, isto é, ele passa a ser insolúvel em termos exatos. Algo dessa natureza pode parecer ultrajante à primeira vista, mas o dilema aparece quando comparamos essa alternativa com a cobertura por bolas fechadas e percebemos que a dificuldade do primeiro é drasticamente menor que a do segundo.

Em mais detalhes, se considerarmos o problema (5.1) com

$$
\mathcal{K}^{\star}:=\left\{x \in \mathbb{R}^{n} \mid g_{i}(x, r, C) \geqslant 0, \forall i \in\{1, \ldots, m+t\}\right\}
$$

no lugar de $\mathcal{K}$, somos amparados pelo Corolário 23 para concluir que $\mathcal{K}^{\star}=\emptyset$ se, e somente se, existem $d \in \mathbb{N}$ e $s_{0}(x), s_{1}(x), \ldots, s_{m+t}(x) \in \Sigma_{n, 2 d}$ tais que

$$
P_{\text {Put }}(x, r, C, s):=s_{0}(x)+s_{1}(x) g_{1}(x, r, C)+\cdots+s_{m+t}(x) g_{m+t}(x, r, C)+1=0 .
$$

Vale comentar que $\operatorname{deg}\left(P_{\text {Put }}(x, r, C, s)\right)=2 d+\max \left\{2, \max \left\{\operatorname{deg}\left(q_{i}\right) \mid i \in\{1, \ldots, t\}\right\}\right\}$ e, por um raciocínio análogo ao anterior, com $s_{i}(x)=[\mathbf{x}]_{d}^{t} S_{i}[\mathbf{x}]_{d}$ e $S_{i} \in \mathcal{S}_{+}^{N(d)}$ para todo $i \in\{1, \ldots, m+t\}$, temos um novo problema:

$\min r$

$$
\begin{aligned}
& \text { sujeito a } \overrightarrow{P_{P u t}}(r, C, S)=\overrightarrow{0} \\
& r \geqslant 0, \\
& S_{i} \succeq 0, \forall i \in\{1, \ldots, m+t\},
\end{aligned}
$$

que, embora seja insolúvel, também é um SDP não linear com $m+t+1$ variáveis matriciais, contra as $2^{m+t}$ variáveis matriciais de (5.3), isto é, o número de esferas da cobertura exerce 
uma influência muito menor nesse caso. Além disso, a variável inteira $b$, que usualmente traz grandes dificuldades computacionais, não existe nessa abordagem.

Outra vantagem de utilizar a cobertura aberta é a simplicidade em calcular exatamente as derivadas de $P_{P u t}(x, r, C, S)$ em relação a todas as variáveis. Abaixo, exibiremos cada uma delas quase explicitamente, definindo $X_{2 d}:=[\mathbf{x}]_{d}[\mathbf{x}]_{d}^{t}$ :

\section{- Derivada no raio:}

$$
\partial P_{P u t}(x, r, C, S) / \partial r=-\sum_{k=1}^{m} S_{k} \cdot X_{2 d}
$$

\section{- Derivada nos centros:}

$$
\partial P_{P u t}(x, r, C, S) / \partial c_{i j}=2\left(c_{i j}-x_{j}\right) S_{i} \cdot X_{2 d}
$$

para $(i, j) \in\{1, \ldots, m\} \times\{1, \ldots, n\}$;

- Derivada nos coeficientes:

$$
\partial P_{P u t}(x, r, C, S) / \partial S_{k}^{(i, j)}=g_{k} X_{2 d}^{(i, j)}
$$

para $k \in\{0, \ldots, m+t\}$ e $(i, j) \in\{1, \ldots, m\} \times\{1, \ldots, n\}$. Além disso, defina $g_{0}:=1$.

Por fim, o vetor de coeficientes de cada uma dessas derivadas constitui sua respectiva linha no jacobiano de $\overrightarrow{P_{P u t}}(r, C, S)$.

Dadas essas vantagens, somos inclinados a questionar se a insolubilidade de (5.5) é mesmo tão ruim assim. Intuitivamente, pode-se imaginar que uma tentativa de resolver (5.5) por um método iterativo, gere uma sequência que se aproxime arbitrariamente da solução, de maneira análoga a minimizar a função $f(x)=x$ no intervalo $(0, \infty)$. Em princípio, não há nada que impeça tal procedimento. Contudo, algumas dificuldades práticas podem aparecer em âmbito computacional, por exemplo, quando uma das variáveis tende ao infinito conforme outras tendem ao ótimo. Isso pode ser ilustrado com um exemplo:

Exemplo 11. Assim como no Exemplo 10, considere dois círculos em $\mathbb{R}^{2}$ definidos pelas desigualdades $p(x, r, c):=\left(x_{1}-c_{1}\right)^{2}+\left(x_{2}-c_{2}\right)^{2}-r \leq 0$ e $q(x):=-p(x, 1, \overrightarrow{0}) \geq 0 . O$ interior do primeiro cobre o segundo quando

$$
K:=\left\{x \in \mathbb{R}^{n} \mid p(x, r, c) \geq 0, q(x) \geq 0\right\}=\emptyset,
$$

o que acontece se, e somente se, existem $d \in \mathbb{N}$ e $s_{0}, s_{1}, s_{2} \in \Sigma_{n, 2 d}$ tais que

$$
P_{P u t}(x, r, c, s):=s_{0}+p s_{1}+q s_{2}+1=0 .
$$

Se $r=1$ e $c=\overrightarrow{0}$, então $p=-q$ e a equação acima torna-se

$$
P_{\text {Put }}(x, 1, \overrightarrow{0}, s):=s_{0}+q\left(s_{2}-s_{1}\right)+1=0 .
$$

Logo,

$$
s_{0}+1=q\left(s_{1}-s_{2}\right) .
$$

Tome uma sequência $\left\{x_{k}\right\}_{k \in \mathbb{N}} \rightarrow x^{\star}$, tal que $\lim _{k \rightarrow \infty}\left\|x_{k}\right\|_{2}=1$ e note que

$$
\lim _{k \rightarrow \infty} s_{0}\left(x_{k}\right)=-1
$$


o que é um absurdo. Logo, a bola com raio 1 e centro $\overrightarrow{0}$ é inviável segundo o positivstellensatz de Putinar para este modelo, como esperado.

Sabendo disso, podemos verificar o que acontece quando tomamos uma sequência de raios $\left\{r_{k}\right\}_{k \in \mathbb{N}} \rightarrow 1$ tal que $r_{k}>1$ para todo $k \in \mathbb{N}$. Para isso, fixemos $c=\overrightarrow{0}$ e um ponto $x \in \mathbb{R}^{2}$ tal que $\|x\|_{2}=1$. Claramente, $x \in V:=\left\{x \in \mathbb{R}^{n} \mid q(x) \leqslant 0\right\}$ e existe uma sequência $\left\{z_{k}\right\}_{k \in \mathbb{N}} \subset \mathbb{R}_{++}$tal que $r_{k}=1+z_{k}$ para todo $k$. Logo,

$$
p\left(x, r_{k}, c\right)=\|x\|_{2}-r_{k}=1-\left(1+z_{k}\right)=-z_{k}
$$

$e$

$$
q(x)=\|x\|_{2}-1=0 .
$$

Sendo assim, como cada $r_{k}$ é viável, isto é, $B\left(\overrightarrow{0}, r_{k}\right)$ cobre $V$, considere $d_{k} \in \mathbb{N}$ e $s_{0}^{k}, s_{1}^{k}, s_{2}^{k} \in$ $\Sigma_{n, 2 d_{k}}$ tais que

$$
s_{0}^{k}+s_{1}^{k} p+s_{2}^{k} q+1=0 .
$$

Nesta situação, iremos supor que $\left\{d_{k}\right\}_{k \in \mathbb{N}}$ é limitada. Avalie essa equação em $x$ para obter

$$
s_{0}^{k}(x)-s_{1}^{k}(x) z_{k}+1=0,
$$

mas note que $z_{k}>0 e$

$$
\lim _{k \rightarrow \infty} s_{1}^{k}(x)=\lim _{k \rightarrow \infty} \frac{s_{0}^{k}(x)+1}{z_{k}}=\infty,
$$

quando $\lim _{k \rightarrow \infty} s_{0}^{k}(x)<\infty$, pois $s_{0}^{k}(x)+1 \geqslant 0$ para todo $k \in \mathbb{N}$ e dizer que $\lim _{k \rightarrow \infty} s_{0}^{k}(x)=$ -1 seria um absurdo.

Como $s_{1}^{k}$ é um polinômio, então a sequência $\left\{s_{1}^{k}\right\}_{k \in \mathbb{N}}$ é ilimitada, se avaliada em $x$. Já que $\left\{d_{k}\right\}_{k \in \mathbb{N}}$ é limitada, podemos afirmar que a norma dos seus coeficientes é também ilimitada, isto é, utilizando sua representação de Gram, temos $\left\|S_{1}^{k}\right\|_{2} \rightarrow \infty$. Um raciocínio análogo pode ser feito se $\lim _{k \rightarrow \infty} s_{0}^{k}(x)=\infty$.

Esse comportamento dos coeficientes SOS notado no Exemplo 11 nos leva a questionamentos sobre sua generalidade e sugere que pelo menos uma variável do problema deve crescer indefinidamente à medida que nos aproximamos da solução. De fato, isso é mesmo verificável, como provaremos a seguir, mas tal resultado demanda um lema geométrico relativamente intuitivo.

Lema 5. Dado um objeto $V:=\left\{x \in \mathbb{R}^{n} \mid q_{1}(x) \geqslant 0, \ldots, q_{t}(x) \geqslant 0\right\}$ compacto, defina

$$
\mathcal{B}(C, r):=\cup_{i=1}^{m}\left\{x \in \mathbb{R}^{n} \mid p_{i}\left(x, r, c_{i}\right)=\left\|x-c_{i}\right\|_{2}^{2}-r<0\right\}
$$

e considere centros $c_{i}$ fixos (não necessariamente ótimos), para $i \in\{1, \ldots, m\}$, e um raio $r^{\star}$ o menor possivel, tal que $\overline{\mathcal{B}\left(C, r^{\star}\right)}$ forma uma cobertura para $V$. Então, existe um ponto $a \in V$ tal que

$$
p_{1}\left(a, r^{\star}, c_{1}\right)=0 \text { e } p_{2}\left(a, r^{\star}, c_{2}\right) \geqslant 0, \ldots, p_{m}\left(a, r^{\star}, c_{m}\right) \geqslant 0 .
$$

Demonstração. Temos dois casos a considerar:

- Se $V \subseteq \mathcal{B}\left(C, r^{\star}\right)$, então a função $d(x, C):=\min \left\{\left\|x-c_{i}\right\|_{2} \mid i \in\{1, \ldots, m\}\right\}$ é contínua em $\mathbb{R}^{n}$ e atinge seu valor máximo $r$ quando restrita a $V$, pois $V$ é compacto. Além disso, por definição, $d(x, C)<r^{\star}$ quando $x \in V$. Logo, $r<r^{\star}$ e $V \subseteq \mathcal{B}(C, r)$, o que é um absurdo ${ }^{1}$.

\footnotetext{
${ }^{1}$ Esta prova elegante da existência do raio $r<r^{\star}$ é creditada ao usuário Umberto P. da plataforma Math. Stack Exchange.
} 
- Caso contrário, existe $a \in V$ tal que $p_{1}\left(a, r^{\star}, c_{1}\right) \geqslant 0, \ldots, p_{m}\left(a, r^{\star}, c_{m}\right) \geqslant 0$. Mas, se as desigualdades fossem todas estritas, a estaria fora da cobertura, o que seria um absurdo. Logo, existe $j \in\{1, \ldots, m\}$ tal que $p_{j}\left(a, r^{\star}, c_{j}\right)=0$ e, sem perda de generalidade, podemos supor que $j=1$.

Observe que $\operatorname{int}\left(\overline{\mathcal{B}\left(C^{\star}, r^{\star}\right)}\right) \neq \mathcal{B}\left(C^{\star}, r^{\star}\right)$.

O ponto em questão no Lema 5 é algo na fronteira da cobertura aberta, incluindo aqueles no interior de $V$. Veja as figuras abaixo para um esclarecimento.

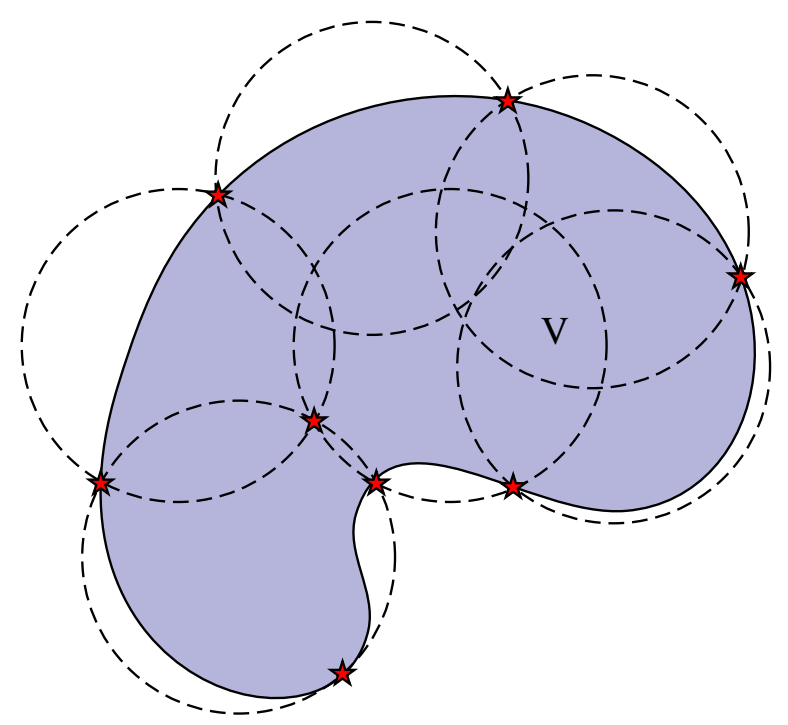

Figura 5.1: Os pontos estrelados satisfazem a descrição do Lema 5.

Pode-se pensar nestes pontos como os primeiros a serem descobertos por $\overline{\mathcal{B}(C, r)}$ quando "o raio diminui", a partir do ótimo. Alternativamente, são os pontos em $V$ que são cobertos por $\overline{\mathcal{B}\left(C, r^{\star}\right)}$, mas não o são por $\mathcal{B}\left(C, r^{\star}\right)$.

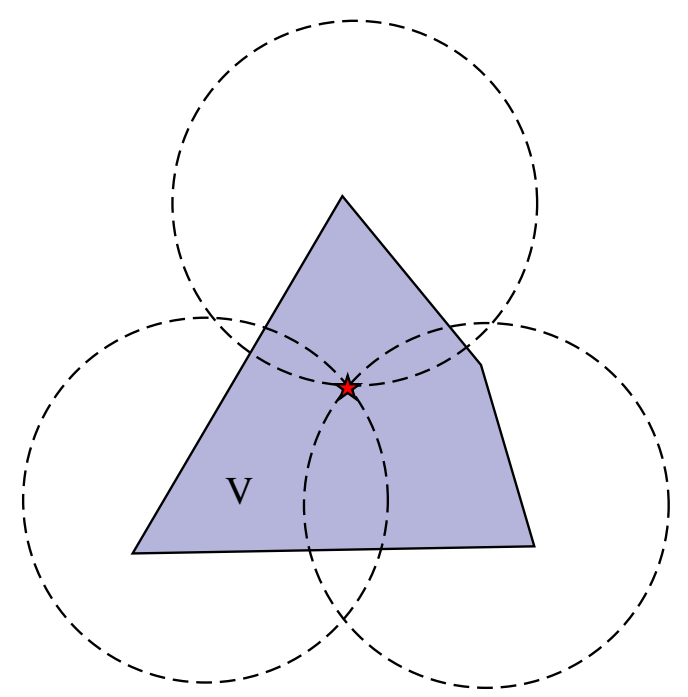

Figura 5.2: O único ponto a satisfazer a descrição do Lema 5 está no interior de $V$.

Dado isso, segue um dos resultados mais importantes deste capítulo, que expõe a característica mais marcante na cobertura com bolas abertas. Ele evidencia que algo de 
especial ocorre nos pontos previstos pelo Lema 5, uma vez que eles são a única diferença entre coberturas abertas e fechadas.

Proposição 30. Fixe $C:=\left\{c_{1}, \ldots, c_{m}\right\} \subset \mathbb{R}^{n}$. Sejam $p_{i}(x, r):=\left\|x-c_{i}\right\|_{2}^{2}-r(i \in$ $\{1, \ldots, m\}), q_{1}(x), \ldots, q_{t}(x)$ polinômios em $\mathbb{R}\left[x_{1}, \ldots, x_{n}\right]$ e $r_{C}^{\star}>0$ o menor raio possível tal que o compacto $V:=\left\{x \in \mathbb{R}^{n} \mid q_{1}(x) \geqslant 0, \ldots, q_{t}(x) \geqslant 0\right\}$ está contido em $\overline{\mathcal{B}\left(C, r_{C}^{\star}\right)}$. Considere uma dada sequência $\left\{r_{k}\right\}_{k \in \mathbb{N}}>r_{C}^{\star}$ tal que $\lim _{k \rightarrow \infty} r_{k}=r_{C}^{\star}$ e seus respectivos certificados de inviabilidade dos conjuntos

$$
\mathcal{K}_{k}:=\left\{x \in \mathbb{R}^{n} \mid p_{1}\left(x, r_{k}\right) \geqslant 0, \ldots, p_{m}\left(x, r_{k}\right) \geqslant 0, q_{1}(x) \geqslant 0, \ldots, q_{t}(x) \geqslant 0\right\},
$$

isto é, inteiros $d_{k} \in \mathbb{N}$ e polinômios $s_{0}^{k}, \ldots, s_{t}^{k}, s_{t+1}^{k}, \ldots, s_{t+m}^{k} \in \Sigma_{n, 2 d_{k}}$, tais que

$$
s_{0}^{k}+s_{t+1}^{k} p_{1}\left(r_{k}\right)+\cdots+s_{t+m}^{k} p_{m}\left(r_{k}\right)+s_{1}^{k} q_{1}+\cdots+s_{t}^{k} q_{t}+1=0 .
$$

Então ou existe $j \in\{0,1, \ldots, t+m\}$ tal que $\lim _{k \rightarrow \infty}\left\|S_{j}^{k}\right\|_{2}=\infty$ ou $\lim _{k \rightarrow \infty} d_{k}=\infty$.

Demonstração. Seja $a \in V$ tal como o Lema 5 descreve, isto é,

$$
p_{1}\left(a, r_{C}^{\star}\right)=0, p_{2}\left(a, r_{C}^{\star}\right) \geqslant 0, \ldots, p_{m}\left(a, r_{C}^{\star}\right) \geqslant 0 .
$$

Mas, como esses valores estão determinados, podemos supor que, para algum índice $v$,

$$
p_{2}\left(a, r_{C}^{\star}\right)=0, \ldots, p_{v}\left(a, r_{C}^{\star}\right)=0, p_{v+1}\left(a, r_{C}^{\star}\right)>0, \ldots, p_{m}\left(a, r_{C}^{\star}\right)>0 .
$$

Então para cada $k \in \mathbb{N}$, seja $z_{k}>0$ tal que $r_{k}=r_{C}^{\star}+z_{k}$. Note que

$$
p_{1}\left(a, r_{k}\right)=\left\|a-c_{1}\right\|_{2}^{2}-r_{k}=\cdots=p_{v}\left(a, r_{k}\right)=\left\|a-c_{v}\right\|_{2}^{2}-r_{k}=r_{C}^{\star}-r_{k}=-z_{k},
$$

por (5.6), agora reescrevendo as equações do P-satz de Putinar avaliadas em $x=a$, temos

$$
\begin{aligned}
& s_{0}^{k}(a)-\left(s_{t+1}^{k}(a)+\cdots+s_{t+v}^{k}(a)\right) z_{k}+s_{t+v+1}^{k}(a) p_{v+1}\left(a, r_{k}\right)+\ldots \\
& \cdots+s_{t+m}^{k}(a) p_{m}\left(a, r_{k}\right)+s_{1}^{k}(a) q_{1}(a)+\cdots+s_{t}^{k}(a) q_{t}(a)+1=0,
\end{aligned}
$$

e isso implica que

$$
\frac{s_{0}^{k}(a)+\sum_{i=v+1}^{m}\left[s_{t+i}^{k}(a) p_{i}\left(a, r_{k}\right)\right]+\sum_{i=1}^{t}\left[s_{i}^{k}(a) q_{i}(a)\right]+1}{z_{k}}=\sum_{i=1}^{v} s_{t+i}^{k}(a) .
$$

Seja $\eta_{k}:=s_{0}^{k}(a)+\sum_{i=v+1}^{m}\left[s_{t+i}^{k}(a) p_{i}\left(a, r_{k}\right)\right]+\sum_{i=1}^{t}\left[s_{i}^{k}(a) q_{i}(a)\right]+1$ o numerador de $(5.7)$. Vamos analisar dois casos:

- Se $\lim _{k \rightarrow \infty} \eta_{k}=0$, então existiria $\bar{k} \in \mathbb{N}$ tal que $\eta_{k}-1<0$ sempre que $k>\bar{k}$. Mas isto seria um absurdo, pois como $a \in V, \eta_{k}-1 \geqslant 0$ para $k$ suficientemente grande. Isso se deve a 5.6 e ao fato de que $\lim _{k \rightarrow \infty} p_{i}\left(a, r_{k}\right)=p_{i}\left(a, r^{\star}\right)>0$ para $i \in\{v+1, \ldots, m\}$.

- Se $\lim _{k \rightarrow \infty} \eta_{k}=\infty$, então existe $j \in\{0,1, \ldots, t\} \cup\{t+v+1, \ldots, t+m\}$ tal que $\lim _{k \rightarrow \infty} s_{j}^{k}(a)=\vec{s}_{j}^{t}[\mathbf{a}]_{2 d_{k}}=\infty$, onde $[\mathbf{a}]_{2 d_{k}}$ é o vetor de monômios $[\mathbf{x}]_{2 d_{k}}$ quando avaliado em $\mathbf{x}=a$ e, pela desigualdade de Cauchy-Schwarz, $\vec{s}_{j}^{t}[\mathbf{a}]_{2 d_{k}} \leqslant\left\|s_{j}^{k}\right\|_{2}\left\|[\mathbf{a}]_{2 d_{k}}\right\|_{2}$. Quando $\left\{\left\|\overrightarrow{s_{j}^{k}}\right\|_{2}\right\}_{k \in \mathbb{N}}$ é limitada, temos $\lim _{k \rightarrow \infty}\left\|[\mathbf{a}]_{2 d_{k}}\right\|_{2}=\infty$ e, portanto, $\lim _{k \rightarrow \infty} d_{k}=$ $\infty$; quando $\left\{d_{k}\right\}_{k \in \mathbb{N}}$ é limitada, $\lim _{k \rightarrow \infty}\left\|\overrightarrow{s_{j}^{k}}\right\|_{2}=\infty$ e isso nos leva a concluir que as matrizes de Gram associadas a $s_{j}^{k}$, denotadas por $S_{j}^{k}$, satisfazem $\lim _{k \rightarrow \infty}\left\|S_{j}^{k}\right\|_{2}=\infty$. Não há mais o que provar nesse caso, pois se ambas as sequências mencionadas são ilimitadas, a conclusão é trivial. 
Quando não ocorre nenhum dos casos acima, nem para subsequência alguma de $\left\{\eta_{k}\right\}_{k \in \mathbb{N}}$, então existe $j \in\{t+1, \ldots, t+v\}$ tal que $\lim _{k \rightarrow \infty} s_{j}^{k}(a)=\infty$ e, por um raciocínio análogo ao segundo caso, ou $\lim _{k \rightarrow \infty}\left\|S_{j}^{k}\right\|_{2}=\infty$ ou $\lim _{k \rightarrow \infty} d_{k}=\infty$ e a demonstração está encerrada.

A Proposição 30 significa que, para toda sequência de raios viáveis tendendo ao ótimo de centros fixos quaisquer, ou a norma de pelo menos uma sequência de matrizes de Gram associadas tende ao infinito ou o grau delas tende ao infinito. Logo, conforme nos aproximamos do raio ótimo, pelo menos uma das variáveis auxiliares que indicam viabilidade cresce arbitrariamente. Tudo isso fere uma das hipóteses principais da teoria de convergência de restauração inexata, pois qualquer sequência convergente para um ponto ótimo será ilimitada.

\section{$5.2 \quad$ Algoritmos}

Mesmo com muitas questões em aberto sobre o modelo (5.5), ele também foi considerado junto ao (5.3) para construir algoritmos. A seguir, exibiremos os algoritmos desenvolvidos com base na teoria de restauração inexata.

\subsubsection{Menor raio para centros fixos}

Tanto em (5.3) quanto em (5.5), fixar $r$ e $C$ dá origem a SDPs lineares de viabilidade. Isso fundamenta um algoritmo simples para construir uma sequência que converge para o melhor raio possível, dados centros fixos $c_{1}, \ldots, c_{m} \in \mathbb{R}^{n}$, um raio inicial factível $r_{f e} \geqslant 0$ e um raio infactível $r_{i n} \geqslant 0$. Esse algoritmo é inspirado na técnica de bissecção, ou seja, a cada iteração ele verifica se o raio médio $r_{\text {med }}$ é viável ou inviável, fazendo $r_{f e} \leftarrow r_{\text {med }}$ no primeiro caso e $r_{i n} \leftarrow r_{m e d}$, no segundo. Este processo é repetido indefinidamente.

Algoritmo 3. Entrada: $V, r_{f e}^{0} \geqslant 0, r_{i n}^{0} \geqslant 0$ e $c_{1}, \ldots, c_{m}$.

1. Defina $r_{m e d}^{k}:=\frac{r_{f e}^{k}+r_{i n}^{k}}{2} e$;

- Se (5.3) (ou (5.5)) tem solução $S_{k}$, então faça $r_{f e}^{k+1} \leftarrow r_{m e d}^{k}$;

- Señ̃o faça $r_{i n}^{k+1} \leftarrow r_{m e d}^{k}$;

2. Faça $k \leftarrow k+1$ e retorne ao passo 1 .

Este algoritmo certamente converge, pois a cerca do raio ótimo diminui a cada iteração e $\lim _{i \rightarrow \infty} r_{f e}^{i}=r^{\star}$.

\subsubsection{Restauração inexata para SDP}

Como o algoritmo desta seção não depende de qual versão do $P$-satz está sendo utilizada, faz-se necessário padronizar a notação dos índices das matrizes $S$. Primeiramente, bastam pequenos passos para verificar que, no P-satz de Stengle, temos $2^{m+t}$ matrizes de Gram disponíveis, enquanto no de Putinar, são $m+t+1$. Por praticidade, denotaremos essas duas quantidades pelo símbolo $\zeta$, que pode ser lastreado de acordo com o contexto. Agora, fixemos também uma indexação para as matrizes de Stengle, isto é, uma 
correspondência bijetiva entre $\{0\} \cup\{\Lambda\}_{\emptyset \neq \Lambda \subseteq\{1, \ldots, m+t\}}$ e $\{0, \ldots, \zeta\}$. Para as de Putinar, essa indexação é trivial.

A teoria de restauração inexata foi estabelecida para um problema qualquer de otimização e, quando

$$
\mathcal{D}:=\mathbb{R}_{+} \times \mathbb{R}^{n \times m} \times \underbrace{\mathcal{S}_{+}^{N(d)} \times \cdots \times \mathcal{S}_{+}^{N(d)}}_{\zeta \text { vezes }}
$$

temos um SDP nas variáveis $(r, C, S)$. Sejam $H(r, C, S):=\overrightarrow{P_{P u t, S t e}}(r, C, S)$ e $J_{H}(r, C, S)$, seu jacobiano.

Para qualquer conjunto de centros $C$, sempre existe um raio viável (suficientemente grande) e, para este raio, existem matrizes $S$ que satisfazem (5.2) (ou (5.4)). Isso compõe a mais simples estratégia de restauração: aumentar o raio e calcular os $S$ apropriados por meio do SDP 5.3 (respec. 5.5) quando eles existirem. Em outras palavras, dado um ponto $(r, C, S)$, deve-se computar $(\bar{r}, \bar{C}, \bar{S})$ tais que

$$
\|H(\bar{r}, \bar{C}, \bar{S})\|_{2} \leqslant \gamma\|H(r, C, S)\|_{2}
$$

mas, para $\bar{C}=C$ e $\bar{r}$ fixo, isso pode ser representado como um SDP linear. Logo, aumentar o raio de maneira conveniente e resolver o mencionado problema é uma das estratégias de restauração. É possível aqui fazer restauração exata ou até mesmo utilizar o Algoritmo 3 para computar, a cada iteração, o melhor raio possível exatamente. Contudo, este é um processo computacionalmente caro.

Primeiramente, vamos definir as seguintes direções: $d_{r}$ no raio, $d_{C}:=\left(d_{c_{1}}, \ldots, d_{c_{m}}\right)$ nos centros e $d_{S}:=\left(d_{S_{0}}, \ldots, d_{S_{\zeta}}\right)$. Além disso, defina $d:=\left(d_{r}, d_{C}, d_{S}\right)$. A fase de minimização tem como um de seus pilares o SDP:

$$
\begin{array}{r}
\min \bar{r}+d_{r} \\
\text { sujeito a } \\
J_{H}(\bar{r}, \bar{C}, \bar{S}) d=\overrightarrow{0} \\
\overline{S_{i}}+d_{S i} \succeq 0, i \in\{0, \ldots, \zeta\} \\
\bar{r}+d_{r} \geqslant 0,
\end{array}
$$

que nada mais é que uma instância do Problema (4.7). A seguir, apresentaremos o primeiro algoritmo de restauração inexata, que é amparado pela teoria da Seção (4.2). Mas, antes disso, definiremos $\Phi(r, C, S, \theta):=\theta r+(1-\theta)\|H(r, C, S)\|_{2}$, e denotaremos por $S \succeq 0$ quando $S_{i} \succeq 0$ para todo $i \in\{0, \ldots, \zeta\}$; o mesmo vale para a expressão $S+d_{S} \succeq 0$.

Algoritmo 4. Iniciamos com pontos $r_{0}, C_{0}:=\left\{c_{1}^{0}, \ldots, c_{m}^{0}\right\}$ e $S^{0}:=\left(S_{0}^{0}, \ldots, S_{\zeta}^{0}\right)$ mais os parâmetros $\varepsilon>0, \theta_{-1}$ e $\gamma \in(0,1)$.

1. Fase de restauração $(k \in \mathbb{N})$ :

- Calcule $H_{k}:=\left\|H\left(r_{k}, C_{k}, S^{k}\right)\right\|_{2}$ e faça $\overline{r_{k}} \leftarrow r_{k}$;

- Encontre um ponto $\left.\overline{S^{k}} \in \underline{\{S} \in\left(\mathcal{S}^{N(d)}\right)^{\zeta} \mid\left\|H\left(\overline{r_{k}}, C_{k}, S\right)\right\|_{2} \leqslant \gamma H_{k}, S \succeq 0\right\}$ e defina $\overline{H_{k}}:=\left\|H\left(\overline{r_{k}}, C_{k}, \overline{S^{k}}\right)\right\|_{2}$. Se nãa for possível, repita este passo com $\overline{r_{k}} \leftarrow \overline{r_{k}}+\Delta_{k}$, sendo $\Delta_{k}=\overline{r_{k-1}}-\overline{r_{k}}$.

\section{Fase de minimização:}


- Calcule uma direção linearmente viável $d_{k}:=\operatorname{argmin}\left\{\overline{r_{k}}+d_{r} \mid d \in T_{k}\right\}$, onde $T_{k}=\left\{d \in \mathbb{R} \times \mathbb{R}^{n \times m} \times\left(\mathcal{S}^{N(d)}\right)^{\zeta} \mid J_{H}\left(\overline{r_{k}}, C_{k}, \overline{S^{k}}\right) d=0, \overline{S^{k}}+d_{S} \succeq 0, \overline{r_{k}}+d_{r} \geqslant 0\right\}$

e $J_{H}$ é uma matriz $\left(\begin{array}{c}n+D \\ D\end{array}\right) \times \overline{N(d)}$. Aqui $D$ é o grau de $P_{\text {Put } / \text { Ste }}$ e $\overline{N(d)}:=$ $n m+1+\zeta\left(N(d)+\left(N(d)^{2}-N(d)\right) / 2\right)$ é o número total de variáveis.

- Calcule

$\theta_{k}:=\max _{\theta}\left\{\theta \in\left[0, \theta_{k-1}\right] \mid \Phi\left(\overline{r_{k}}, C_{k}, \overline{S^{k}}, \theta\right)-\Phi\left(r_{k}, C_{k}, S^{k}\right) \leqslant \frac{1-\gamma}{2}\left(\overline{H_{k}}-H_{k}\right)\right\}$,

isto é,

$$
\theta_{k}=\min \left\{\theta_{k-1}, \frac{(\gamma+1)\left(H_{k}-\overline{H_{k}}\right)}{2\left(\overline{r_{k}}-r_{k}-\overline{H_{k}}+H_{k}\right)}\right\}
$$

- Calcule

$\alpha_{k}:=\max _{\alpha}\left\{\alpha \in[0,1] \mid \Phi\left(\left(\overline{r_{k}}, C_{k}, \overline{S^{k}}\right)+\alpha d_{k}, \theta_{k}\right)-\Phi\left(r_{k}, C_{k}, S^{k}, \theta_{k}\right) \leqslant \frac{1-\gamma}{2}\left(\overline{H_{k}}-H_{k}\right)\right\}$.

Uma das formas é definir $\alpha_{k} \leftarrow 1$ e, caso o critério não seja satisfeito, fazer $\alpha_{k} \leftarrow \alpha_{k} / 2$ até ser satisfeito (busca linear).

- $F a c ̧ a ~\left(r_{k+1}, C_{k+1}, S^{k+1}\right) \leftarrow\left(\overline{r_{k}}, C_{k}, \overline{S^{k}}\right)+\alpha_{k} d_{k}$.

3. Critério de parada: se $\left\|\left(r_{k}, C_{k}\right)-\left(r_{k}, C_{k-1}\right)\right\|_{2}>\varepsilon$, volte ao Passo 1. Senão, defina $C^{\star} \leftarrow C_{k}, r^{\star} \leftarrow r_{k}$ e retorne ambos.

\subsubsection{Sobre a implementação}

O programa foi implementado em MATLAB, gerando uma entrada para o SeDuMi através do $Y A L M I P$. As operações são feitas com polinômios em variáveis simbólicas e seus coeficientes são extraídos por meio da função coefficients. Para atribuir valores às variáveis, foi utilizada a função replace. Sobre a estrutura, primeiro é computado um modelo simbólico para o P-satz e, no caso de Putinar, também um jacobiano simbólico, que são calculados uma única vez e cujos valores são atribuídos durante a execução. Em seguida, os centros iniciais são calculado aleatoriamente e, após isso, é aplicado o Algoritmo 3 para computar um raio viável inicial e suas respectivas matrizes de Gram. Esses são os dados de entrada para a função uniraioinexrest (Algoritmo 4), junto a uma série de parâmetros que compõem a restauração inexata. Ao final, obtemos como resposta o raio ótimo, os centros ótimos, as matrizes de Gram associadas, uma tabela com os dados da execução e uma representação gráfica dos iterandos.

Lidando com o positivstellensatz de Putinar e tendo em mente a Proposição 30, é esperado que $\left\|d_{S}\right\|_{2}$ seja exageradamente grande, fazendo com que todas as outras direções também tenham tamanhos maiores que o necessário. Assim, limitá-los de maneira apropriada é uma forma de economizar iterações na busca linear. Isso é feito acrescentando a restrição

$$
\left\|d_{S}\right\|_{2} \leqslant \sigma\|S\|_{2}
$$

$\operatorname{com} \sigma \in[0,1]$, a (5.9). Ao nos aproximarmos da solução, notamos que o passo nos centros e no raio tende a ficar pequeno. Logo, este parâmetro $\sigma$ é incrementado em função das iterações. Da mesma forma, quanto maior o parâmetro $\gamma$, maior o passo aceito, em geral. Então, este parâmetro também é incrementado da mesma forma que $\sigma$. 
Com o positivstellensatz de Stengle, foi observado que quando menos viável o ponto, mais imprecisa será a solução de (5.9), de modo a impedir o progresso do algoritmo. Para evitar complicações, deve-se dar uma grande preferência pela viabilidade nesse caso, o que implica em caminhar a passos curtos. Logo, o parâmetro $\gamma$ é constante e pequeno quando tratamos do $P$-satz de Stengle. A restrição (5.10) foi mantida, mas o parâmetro $\sigma$ também se torna constante.

Vale ressaltar que nos propusemos a oferecer uma ideia alternativa à abordagem usual para um problema clássico e, neste trabalho, não priorizamos a velocidade de execução, nem otimização de código. Por isso, também não foi feita uma comparação prática direta com as técnicas baseadas em discretizações.

O computador utilizado nos testes utiliza um sistema operacional de 64 bits, com 4GB de memória RAM e um processador Intel Core i5-5200 de 2.20GHz. A versão do MATLAB utilizada para a implementação é o R2015a com a "Symbolic Math Toolbox". 


\subsection{Testes computacionais (Putinar $+\mathrm{RI}$ )}

Aqui estão compilados alguns testes envolvendo o modelo (5.5). Alguns dos casos têm a solução exata conhecida e outros, não. Fatores como o número de iterações, tempo de execução, erros e parâmetros também são mencionados e, apesar de ser possível aplicar o algoritmo para dimensões maiores, apenas o caso bidimensional será apresentado.

\subsubsection{Um círculo com círculos abertos}

Ao longo desta divisão do trabalho, $V$ é o círculo unitário centrado na origem (em preto) e $\mathcal{B}$ é uma coleção de $m$ círculos com raio e centros variáveis (em lilás, amarelo e azul). Nos quatro testes a seguir $m=1,2,3$ e 6 , respectivamente.

O primeiro teste consiste em cobrir $B(\overrightarrow{0}, 1)$ com apenas um círculo e teve como entrada um centro aleatório no quadrado $[0,1] \times[0,1]$, para constar, $c^{0}=[0.8147,0.9058]$ e o melhor raio para este círculo, calculado pelo Algoritmo $3, r_{0}=2.2189$, que também retorna os valores de $S^{0}$ associados (em 24.3s). O tempo para criar as variáveis e equações do $P$-satz foi $3.7 \mathrm{~s}$, para criar o jacobiano simbólico, foi de $2.5 \mathrm{~s}$ e, após 597 iterações e $1606.1 \mathrm{~s}$ com parâmetro $\gamma=0.9832$, foram retornados o centro $c^{\star}=(0.4252,0.2547) \times 10^{-12}$ e o raio $r^{\star}=1.00039$, caracterizando erro de $3.9 \times 10^{-4}$ em relação à sua solução exata. Isto é, o erro do raio é discrepantemente maior que o erro dos centros, mas o tamanho do passo nas últimas iterações era da ordem de $10^{-9}$ para o raio e de $10^{-17}$ para os centros. A norma de $H$ na solução (isto é, sua viabilidade) é $0.86 \times 10^{-6}$ e $d=2$.

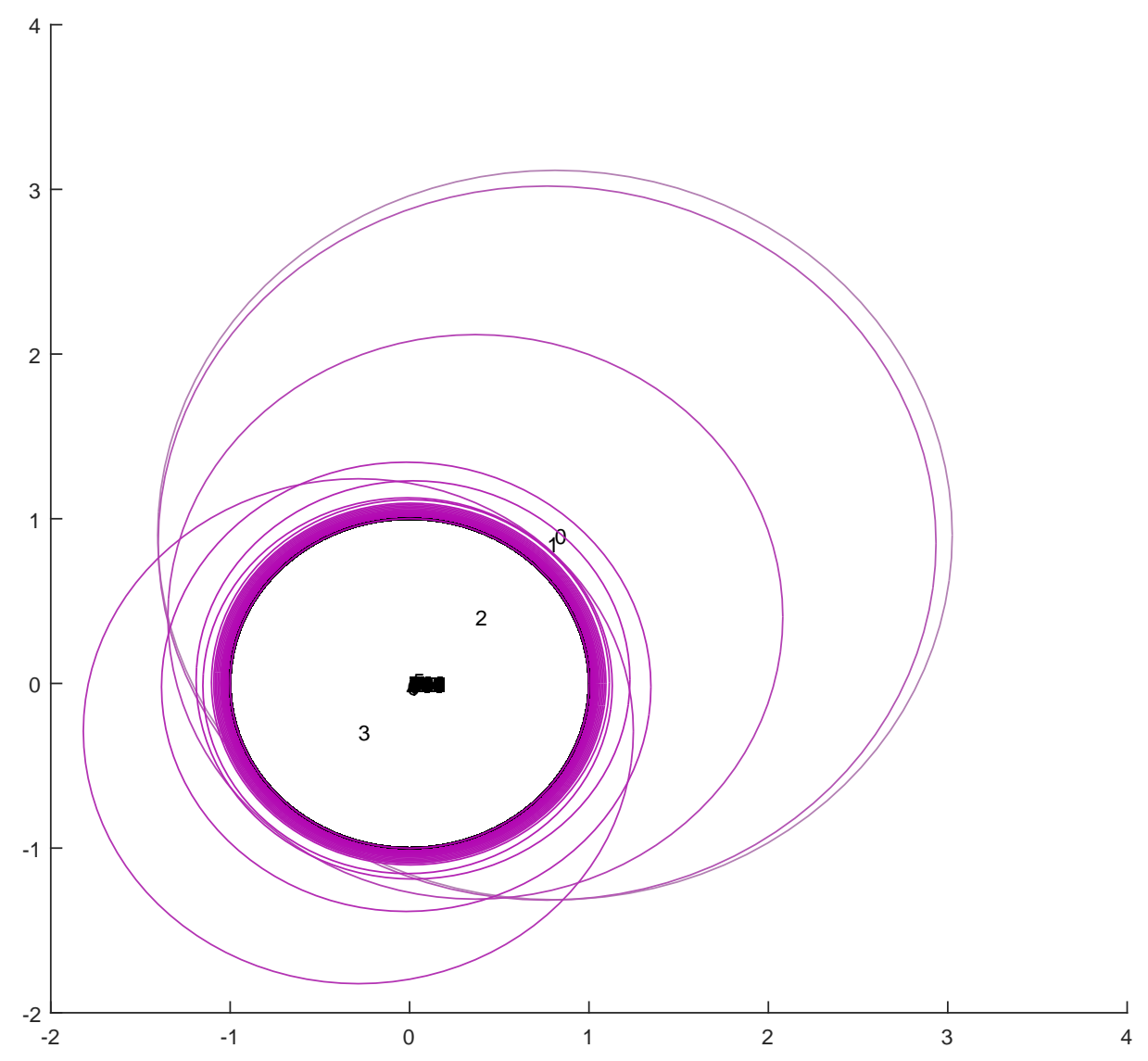

Figura 5.3: Iterandos do primeiro teste cobrindo um círculo. 
O segundo teste, com dois círculos cobrindo $B(\overrightarrow{0}, 1)$, contou com a mesma metodologia do primeiro: teve como entradas os centros $c_{1}^{0}=(0.127,0.9134), c_{2}^{0}=(0.6324,0.0975)$ e o raio $r_{0}=2.6738$. O tempo para criar o modelo foi de $3.3 \mathrm{~s}$, para o jacobiano foram $12.1 \mathrm{~s}$, para executar o Algoritmo 3 foram 22.9s e, para a solução, foram 3139.3s. A norma de $H$ na solução é de $0.627 \times 10^{-3}$ e foram necessárias 171 iterações para convergir para o raio $r^{\star}=1.0018$ e centros $c_{1}^{\star}=-c_{2}^{\star}=(0.007,0)$ (um ótimo é $r^{\star}=1$ e $c_{1}^{\star}=c_{2}^{\star}=\overrightarrow{0}$ ) com tamanho de passo final na ordem de $10^{-5}$ tanto nos centros, quanto no raio. Além disso, $\gamma=0.9415$ e $d=2$.

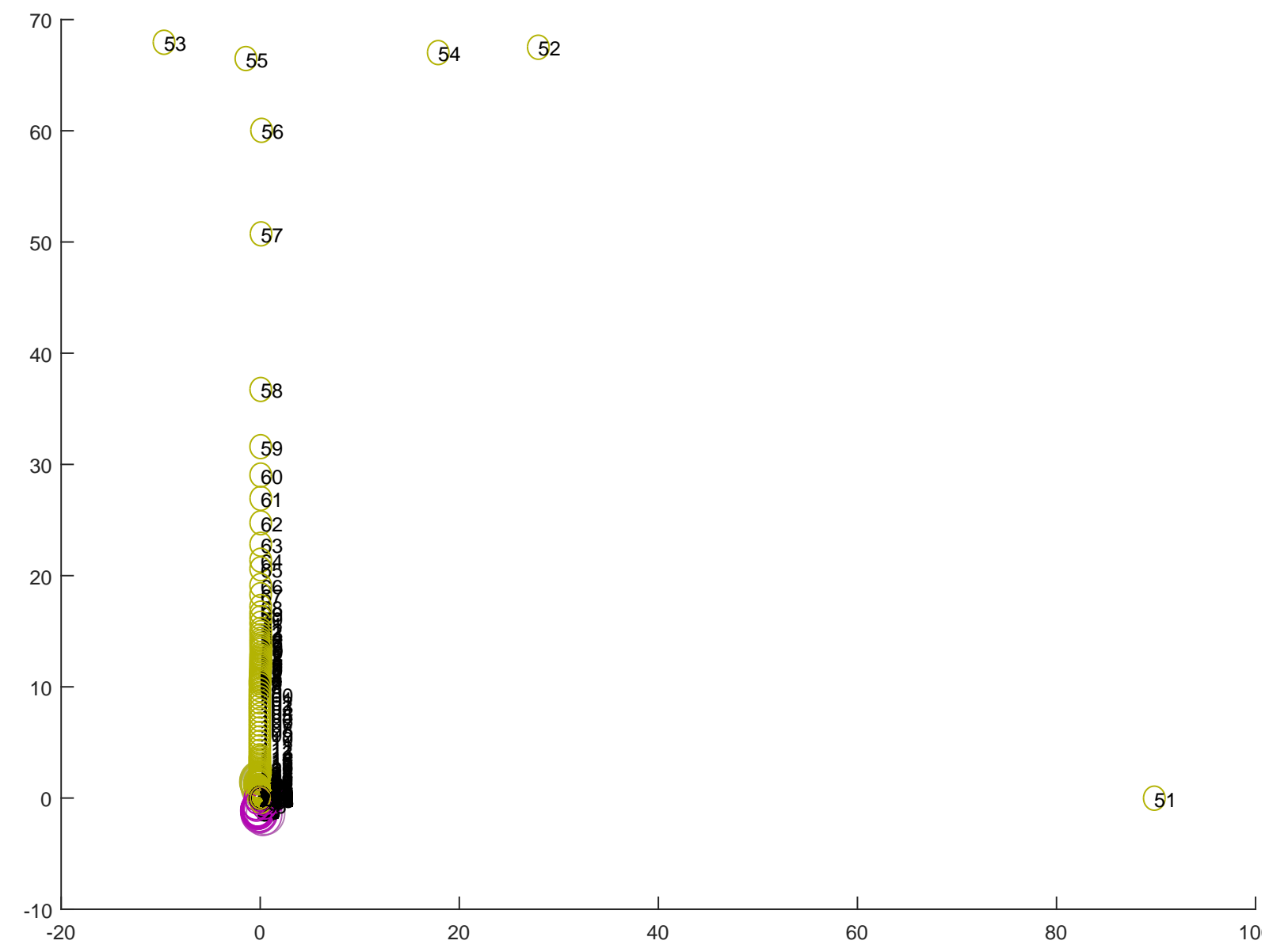

Figura 5.4: Iterandos do segundo teste cobrindo um círculo.

Existem infinitas soluções para esse caso, além das rotações do ótimo encontrado. Se um dos círculos tiver centro em $\overrightarrow{0}$ e raio 1 , o outro pode ser arbitrário. Isso explica o motivo da sequência lilás convergir para o círculo unitário e a sequência amarela divergir inicialmente. Porém, o fato curioso é que ambos os círculos convergiram para a origem posteriormente, o que não seria necessário. 


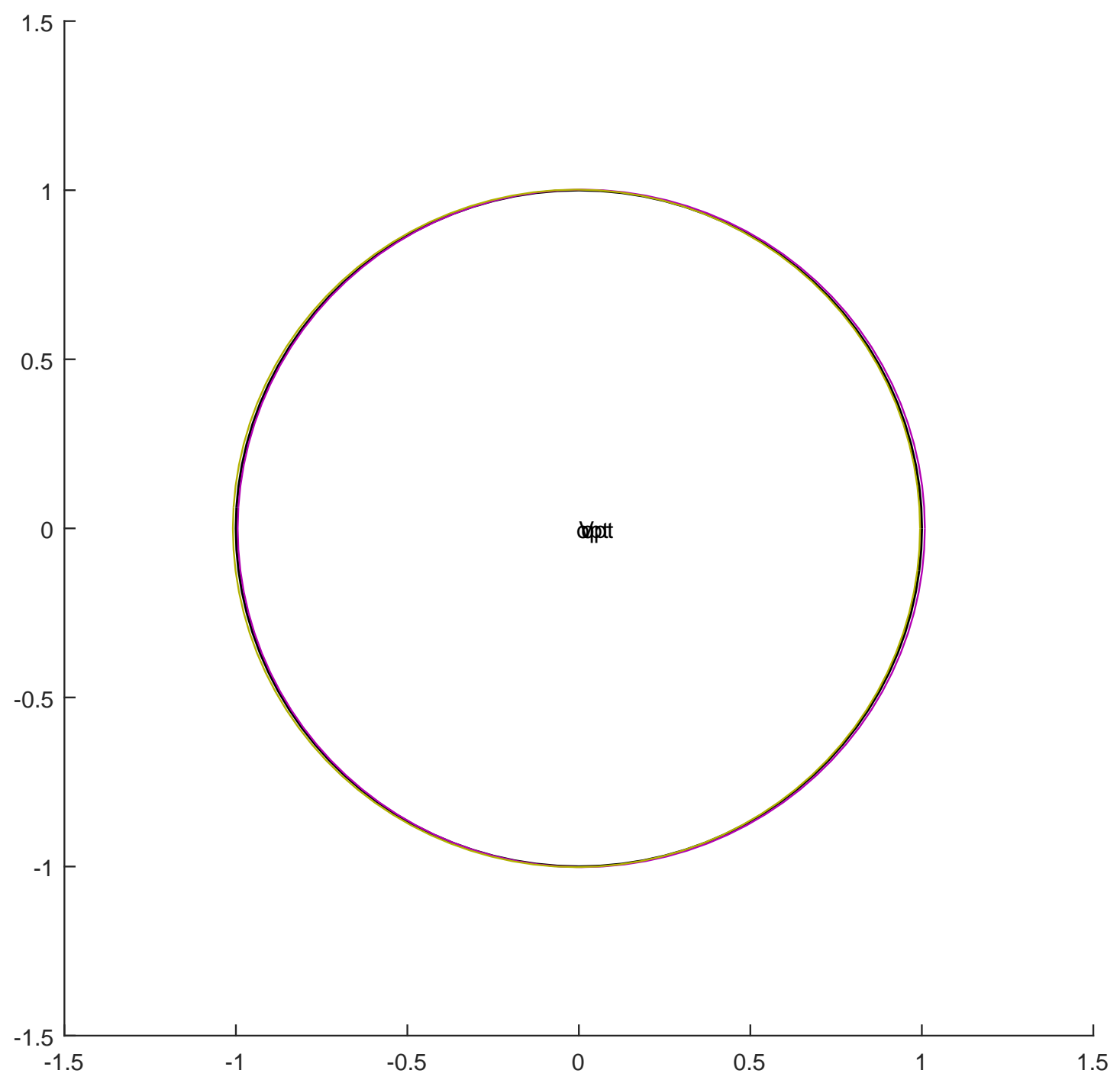

Figura 5.5: Solução do segundo teste cobrindo um círculo. 
O terceiro teste foi feito com três círculos cobrindo $B(\overrightarrow{0}, 1)$, com centros dados inicialmente por $c_{1}^{0}=(0.2785,0.5469), c_{2}^{0}=(0.9575,0.9649)$ e $c_{3}^{0}=(0.1576,0.9706)$, raio inicial $r_{0}=2.6094$, parâmetro $\gamma=0.9921$ e grau $d=4$ para os polinômios SOS envolvidos. O tempo para criar o modelo, o jacobiano e a primeira execução da bissecção foi de 49.6s, 44.7s e 45.6s, respectivamente. A solução encontrada, após 1263 iterações e $38354.4 \mathrm{~s}$, foram os centros $c_{1}^{\star}=(0.4332,0.25), c_{2}^{\star}=(-0.4332,0.25), c_{3}^{\star}=(0,-0.5003) \mathrm{e}$ o raio $r^{\star}=0.867871$ (o raio ótimo é $\sqrt{0.75}=0.866025$, logo o erro é 0.001845 ), com a norma de $H$ igual a $0.6679 \times 10^{-3}$. Contudo, isso está relacionado com as normas das matrizes $S_{i}$ e a cobertura é estritamente viável.

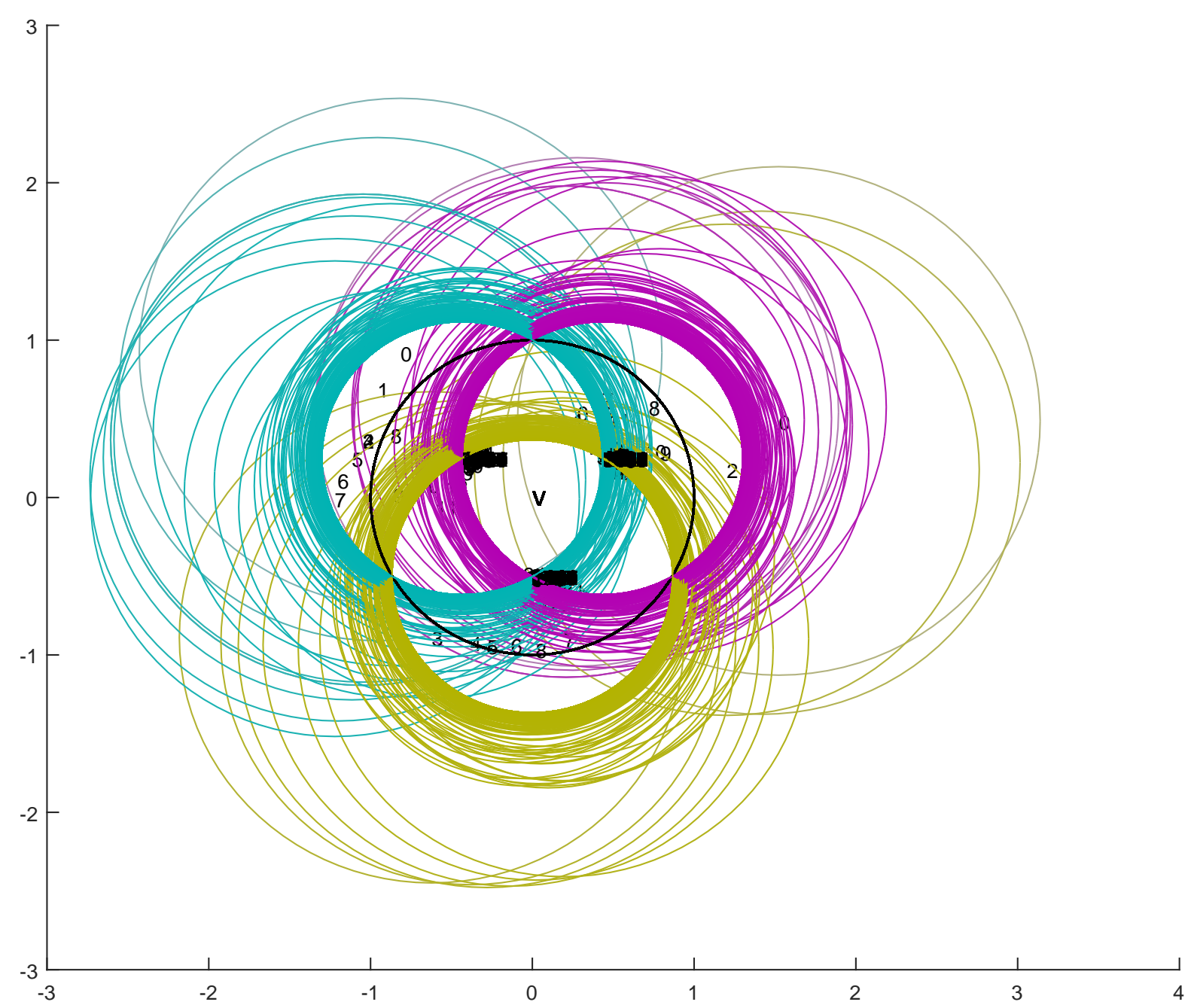

Figura 5.6: Iterandos do terceiro teste cobrindo um círculo.

Vale ressaltar que cobrir figuras "muito simétricas" é mais difícil que as que não compartilham dessa característica. Por exemplo, no caso do círculo, existem infinitas soluções e, próximo a uma delas, as direções nos centros tomam um rumo rotativo. Este problema pode ser contornado em algumas instâncias fixando uma coordenada de um dos centros, limitando a simetria. 


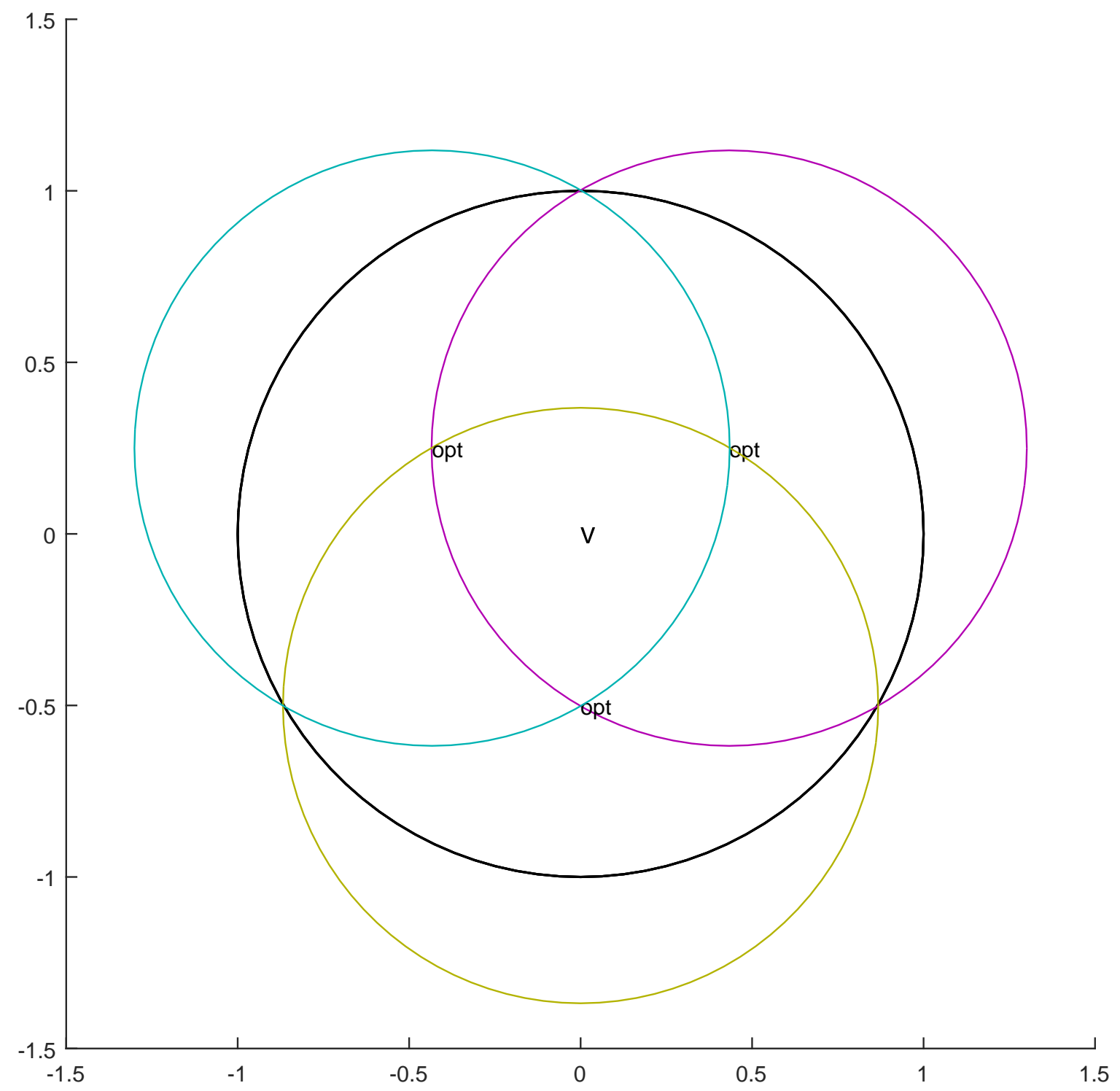

Figura 5.7: Solução do terceiro teste cobrindo um círculo. 
O quarto teste foi feito com seis círculos com centros iniciais (não aleatórios, devido à sensibilidade do algoritmo aos dados entrada) em $c_{1}^{0}=-c_{2}^{0}=(0.5,0.5), c_{3}^{0}=-c_{4}^{0}=$ $(0,0.5), c_{5}^{0}=-c_{6}^{0}=(0.5,-0.5)$, raio $r_{0}=0.7126$ e $d=4$. Foram necessários $39.9 \mathrm{~s}$ para criar as variáveis, $55.4 \mathrm{~s}$ para calcular o jacobiano e 37.1s para calcular o raio inicial. Após 1882 iterações e 93854.6 s, o algoritmo encontrou a solução

$$
\begin{gathered}
c_{1}^{\star}=(0.4797,0.2785) \\
c_{2}^{\star}=(0.4797,-0.2785) \\
c_{3}^{\star}=(0,-0.8241)
\end{gathered}
$$

$$
\begin{gathered}
c_{4}^{\star}=(0,0.5601) \\
c_{5}^{\star}=(-0.7159,0.4087) \\
c_{6}^{\star}=(0.7159,0.4088)
\end{gathered}
$$

e raio $r^{\star}=0.5585696$ (o ótimo real é 0.5561735 , conforme provado por K. Bezdek em 1979 [Fri18]. Logo, o erro absoluto é 0.0023961).

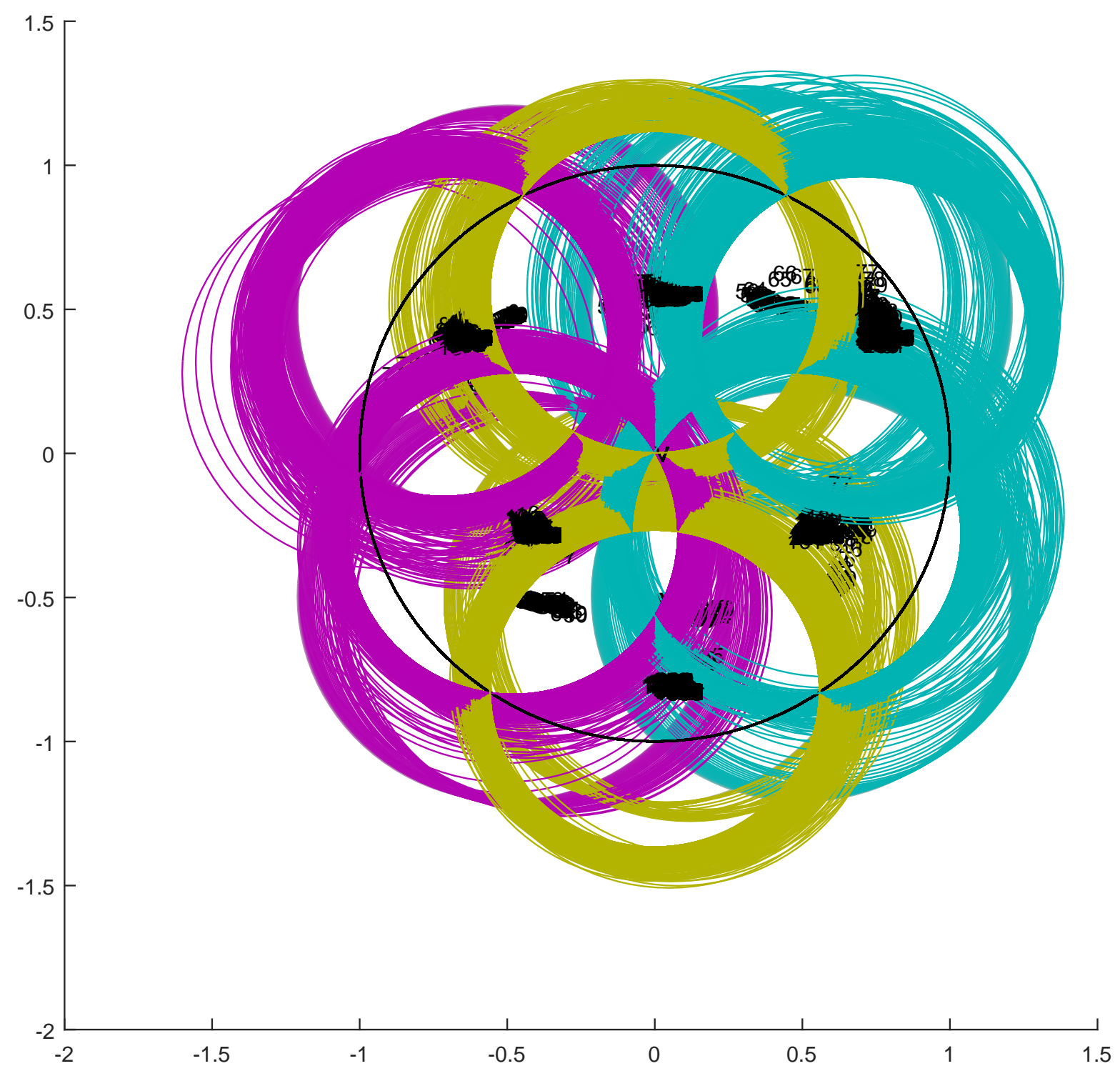

Figura 5.8: Iterandos do quarto teste cobrindo um círculo. 


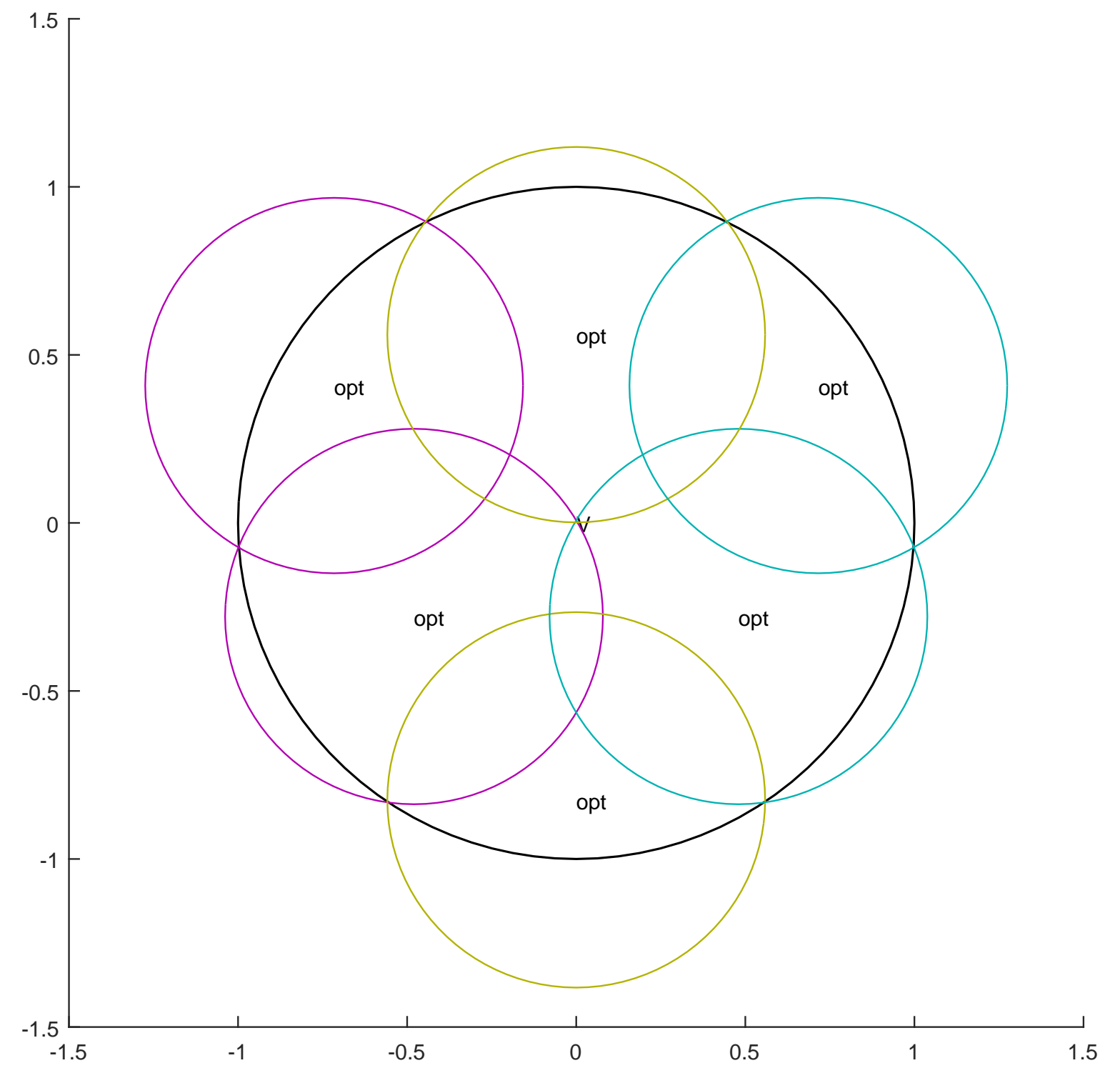

Figura 5.9: Solução do quarto teste cobrindo um círculo.

Um fato interessante a ser comentado é que a solução computada e exibida neste trabalho, possui uma estrutura diferente da solução de Bezdek (veja [Fri18]), que inclusive também fomos capazes de encontrar (com $r^{\star}=0.560178$, logo o erro foi de 0.004) fornecendo uma cobertura inicial diferente. Veja abaixo a dita solução:

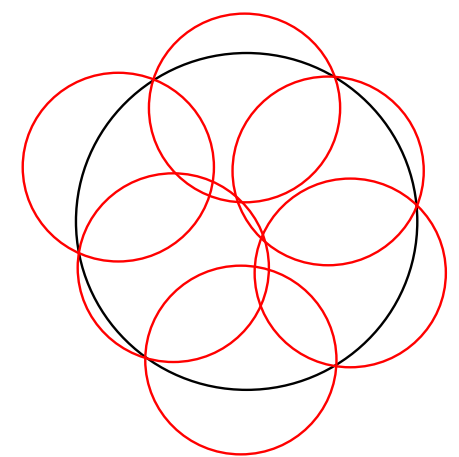

Figura 5.10: Solução de K. Bezdek para seis círculos. 


\subsubsection{Um coração com círculos abertos}

A segunda figura que tentamos cobrir é $\boldsymbol{\vee}:=\left\{x \in \mathbb{R}^{2} \mid\left(x_{1}^{2}+x_{2}^{2}-1\right)^{3}-x_{1}^{2} x_{2}^{3} \leqslant 0\right\}$ utilizando um, três e sete círculos. Fizemos este caso para mostrar que esta técnica não se limita apenas a regiões convexas, mas infelizmente não existe uma solução provadamente correta para ele. Logo, informações sobre o erro não estão disponíveis. Lembrando que este caso é mais simples que o anterior, pela finitude das soluções.

O primeiro teste foi cobrir $\boldsymbol{\nabla}$ com apenas um círculo, e o resultado encontrado após 1141 iterações e $47535.1 \mathrm{~s}$, foi $c^{\star}=(0,0.227859)$ e $r^{\star}=1.263672$, com viabilidade $\|H\|_{2}=$ $0.9124 \times 10^{-4}$ e o tamanho do passo, tanto no centro quanto no raio, na ordem de $10^{-6}$. Este caso encontrou grandes dificuldades para convergir perto da solução, mas enquanto longe, houve facilidade.

Todos os testes com $\checkmark$ foram executados com $d=6$.

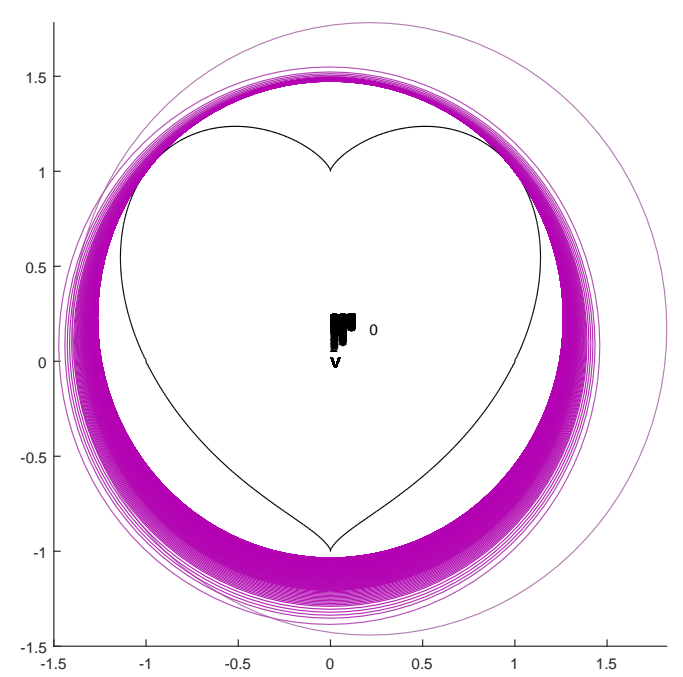

Figura 5.11: Iterandos do primeiro teste cobrindo um coração.

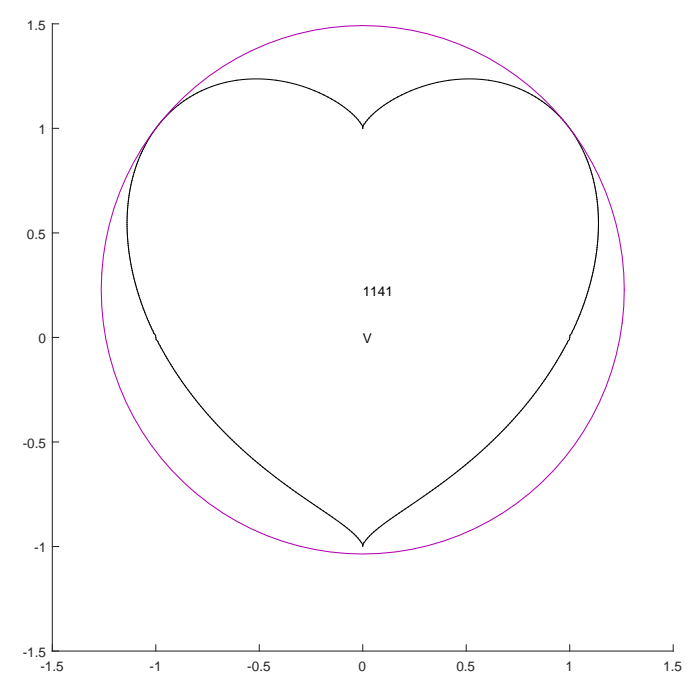

Figura 5.12: Solução do primeiro teste cobrindo um coração. 
O segundo teste terminou em 800 iterações $(30324.9 \mathrm{~s})$ e resultou nos centros $c_{1}^{\star}=$ $(-0.0005,-0.3054), c_{2}^{\star}=(0.4989,0.4358)$ e $c_{3}^{\star}=(-0.4984,0.4358)$ e raio $r^{\star}=0.806536$, com norma de $H$ igual a $8.1 \times 10^{-5}$ e tamanho de passo no centro da ordem de $10^{-5}$ e no raio, de $10^{-6}$. Assim como todos os outros testes, $\gamma \in(0.98,0.999)$.

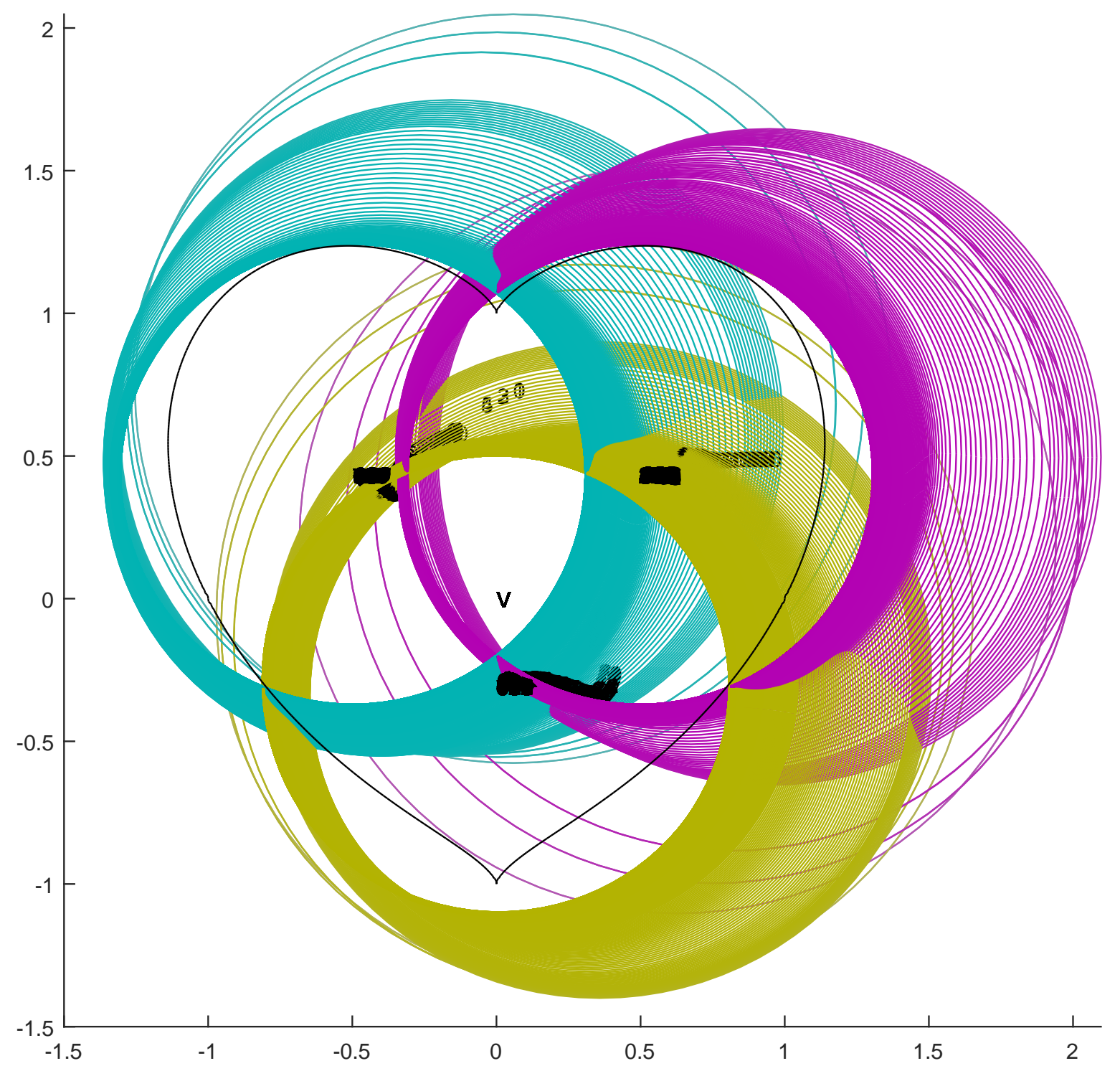

Figura 5.13: Iterandos do segundo teste cobrindo um coração.

Este caso se mostrou especialmente amigável à convergência. Pode-se inferir que a causa disso é a falta de simetria na rotação. Abaixo está a solução deste problema. 


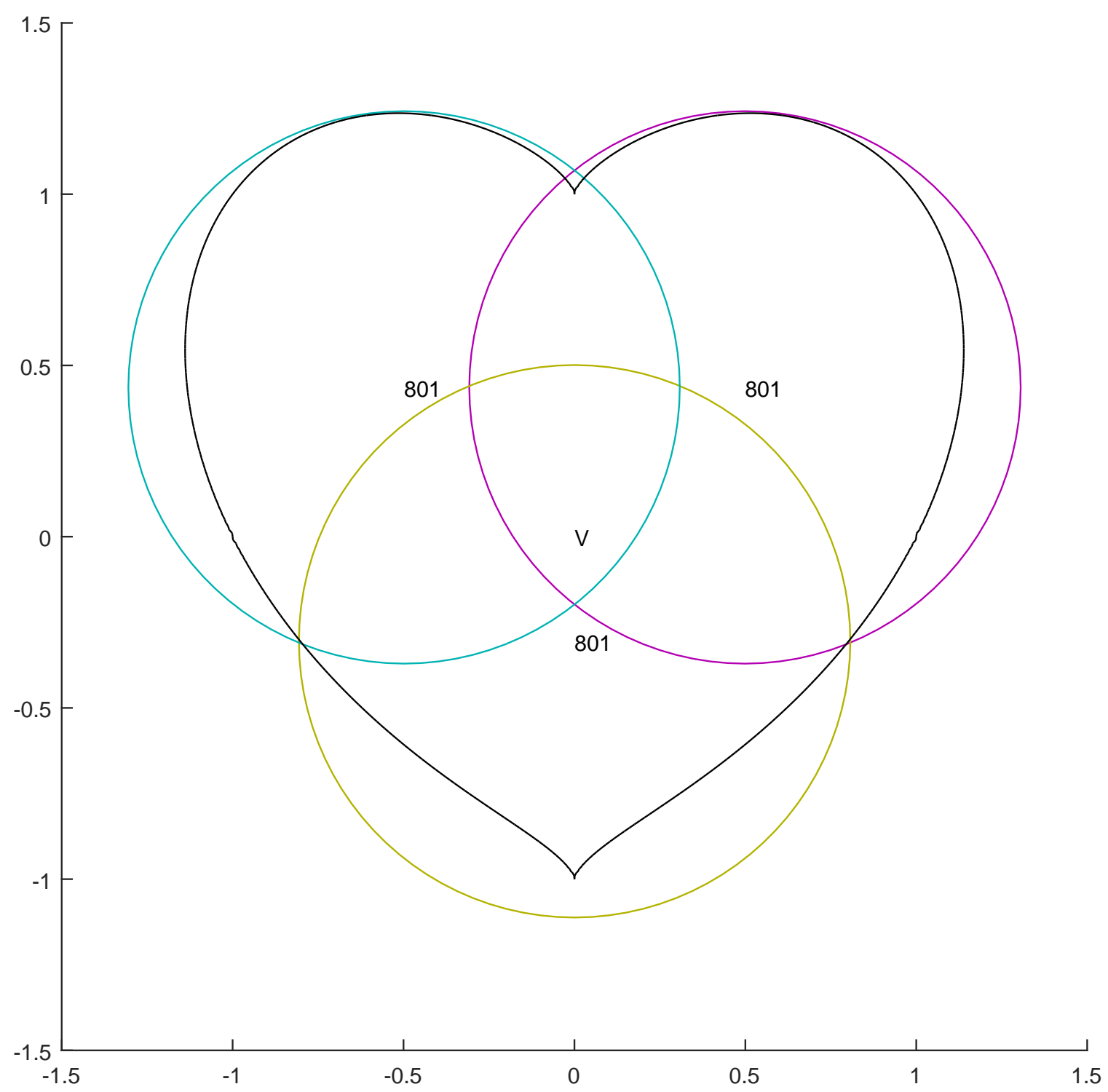

Figura 5.14: Solução do segundo teste cobrindo um coração. 
O terceiro teste, com sete círculos, levou 413 iterações e 46693.1s para terminar, e retornou a cobertura

$$
\begin{gathered}
r^{\star}=0.5523586 \\
c_{1}^{\star}=(0,-0.5638) \\
c_{2}^{\star}=(-0.0001,1.1556) \\
c_{3}^{\star}=(-0.7473,-0.0723)
\end{gathered}
$$

$$
\begin{gathered}
c_{4}^{\star}=(0.7474,-0.0722) \\
c_{5}^{\star}=(0,0.3557) \\
c_{6}^{\star}=(-0.785,0.7608) \\
c_{7}^{\star}=(0.785,0.7608)
\end{gathered}
$$

com $\|H\|_{2}=0.23 \times 10^{-5}$ e tamanho de passo, tanto nos centros, quanto no raio, da ordem de $10^{-7}$.

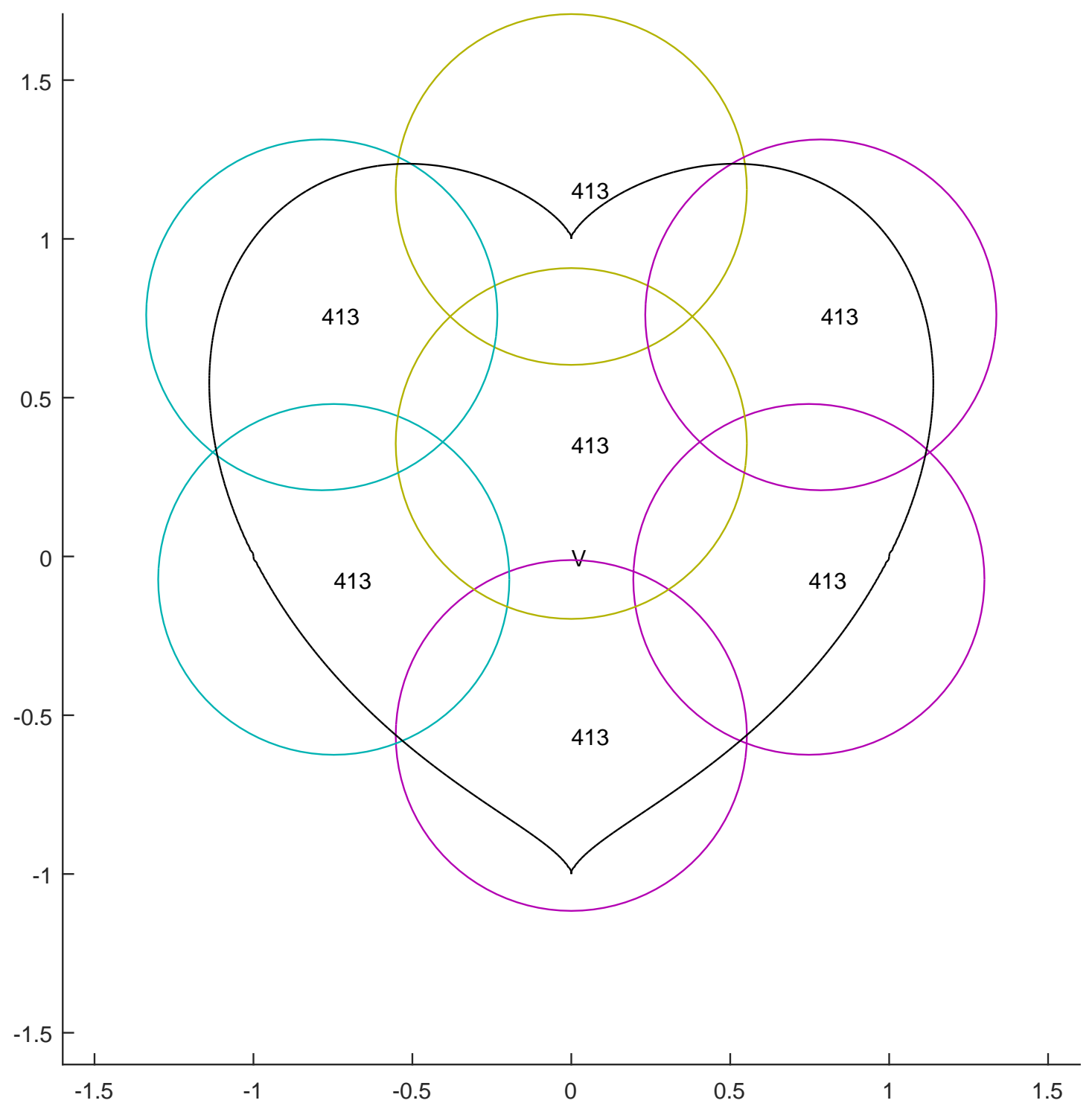

Figura 5.15: Solução do terceiro teste cobrindo um coração. 


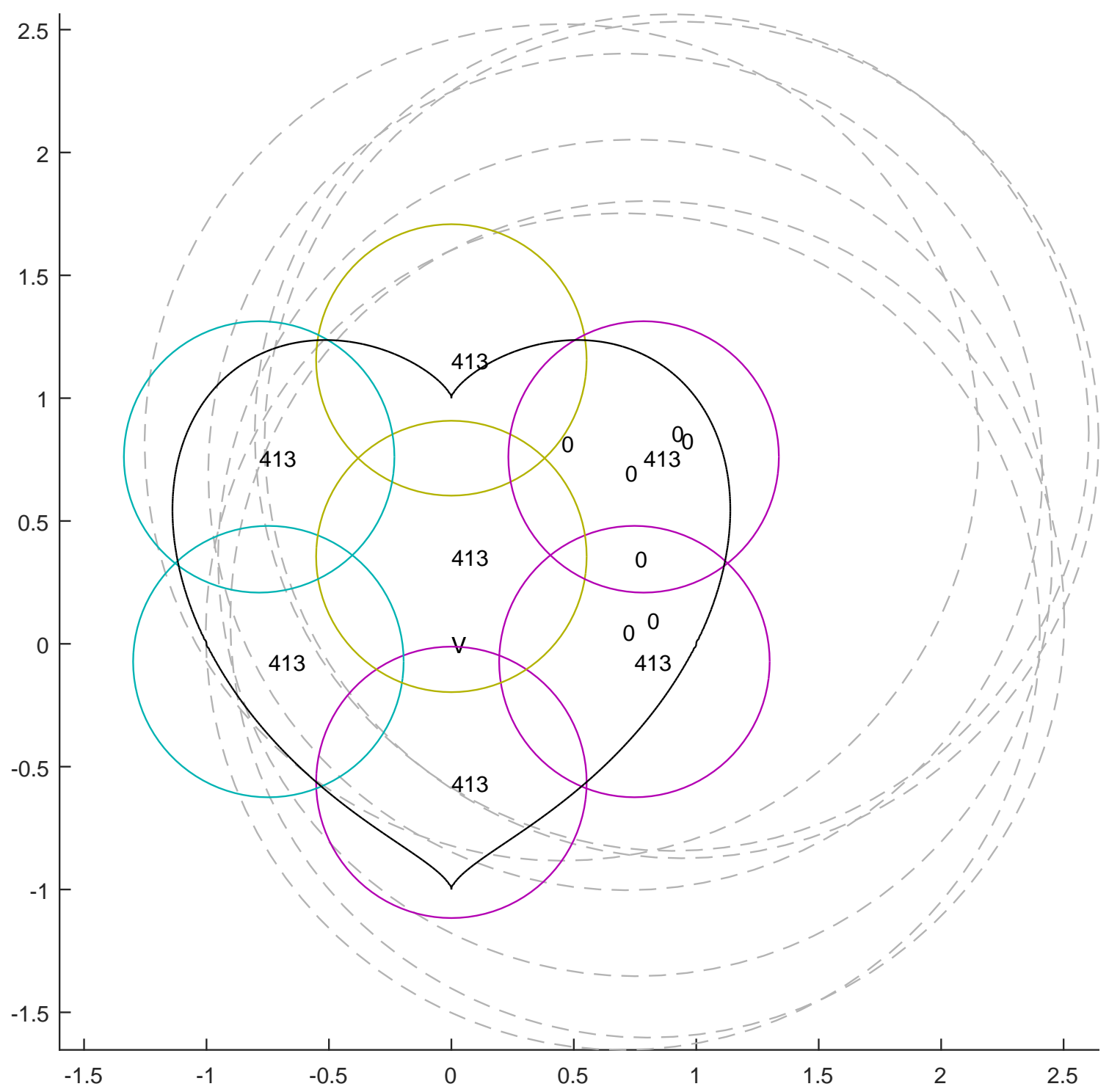

Figura 5.16: Iteração inicial e final do terceiro teste cobrindo um coração. 


\subsection{Infinitos e coberturas abertas}

Em vista da Proposição 5, é esperado que as normas das matrizes de Gram dos coeficientes polinomiais SOS de Putinar crescam indefinidamente conforme nos aproximamos de um raio ótimo. De fato, isso pode ser verificado computacionalmente; fixando $V=B(\overrightarrow{0}, 1)$ e $\mathcal{B}=B(\overrightarrow{0}, r) \subseteq \mathbb{R}^{2}$, cada raio $r>1$ possui matrizes de Gram $S_{0}(r), \ldots, S_{3}(r)$ associadas que garantem sua viabilidade como cobertura, mas a priori elas não são únicas. Sendo assim, considere os $S_{i}$ de menor norma possível e defina a função $\|S(r)\|_{2}:=\max \left\{\left\|S_{i}(r)\right\|_{2} \mid i \in\{0, \ldots, 3\}\right\}$. Desta forma, o mapa $r \mapsto\|S(r)\|_{2}$ está bem definido. Eis um gráfico dele:

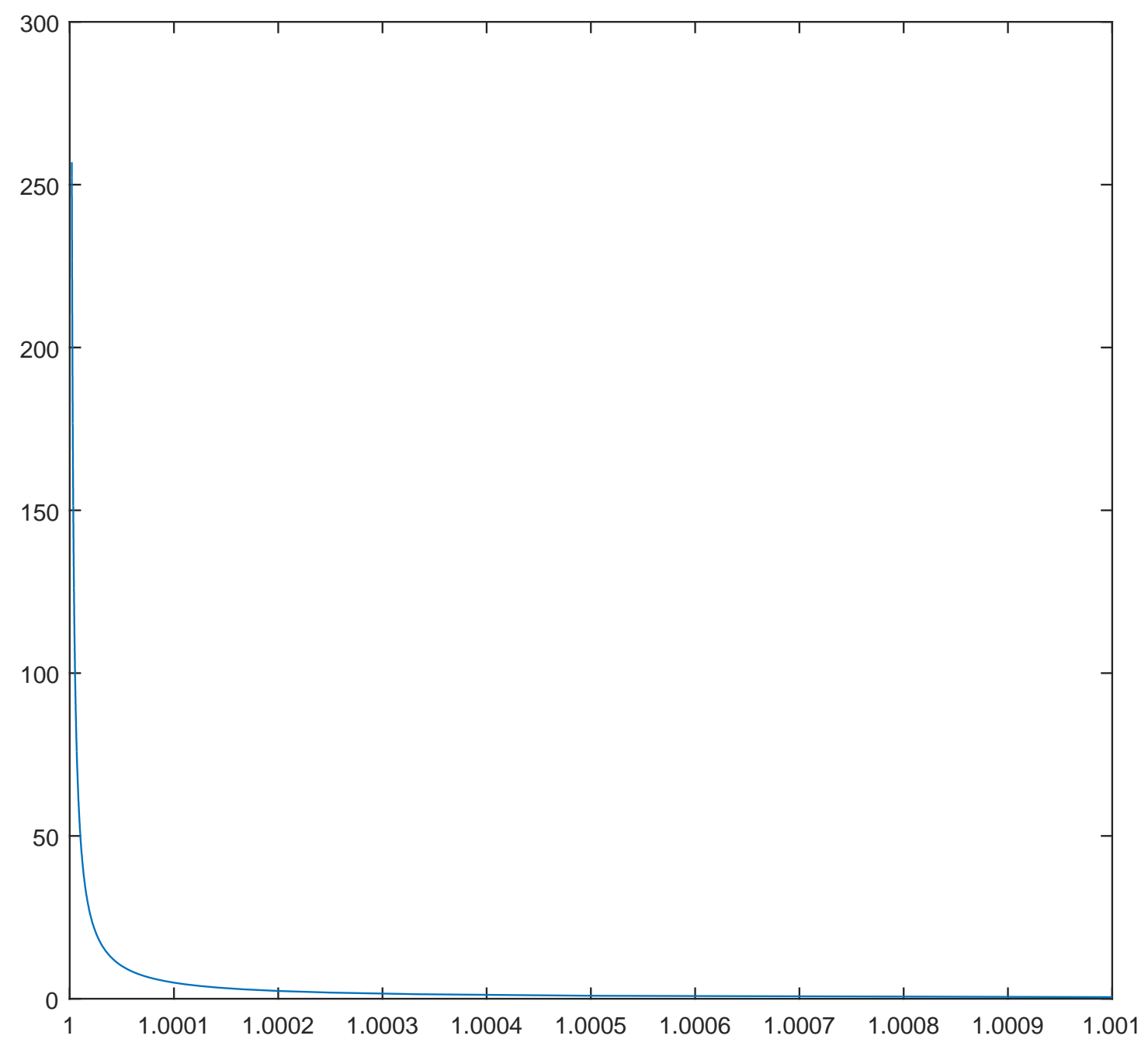

Figura 5.17: Gráfico de $r \mapsto\|S(r)\|_{2}$ no problema de cobertura com $V=B(\overrightarrow{0}, 1)$ e $m=1$.

Devido a imprecisões computacionais inerentes aos SDPs, o raio $r=1.0000021$ foi o menor raio viável encontrado. Note que as variáveis $S$ não têm participação na função objetivo, mas servem apenas para caracterizar a região factível. Além disso, sua quantidade é, em geral, muito maior que a de variáveis "importantes" e o fato de seus valores tenderem ao infinito gera inúmeras dificuldades práticas, embora teoricamente não haja problema algum. Tendo em mente que $\left(r^{\star}, c^{\star},\left\|S\left(r^{\star}\right)\right\|_{2}\right)=(1, \overrightarrow{0}, \infty)$ representa, de alguma forma, a solução do problema, pode-se fazer uma pequena analogia com um exemplo. 
Exemplo 12. Considere o seguinte problema de otimização:

$$
\begin{array}{cc}
\min x \\
\text { sujeito a } x y=1 \\
x \geqslant 0,
\end{array}
$$

que não tem solução formal, mas claramente a sequência viável $\left(x_{k}, y_{k}\right):=(1 / k, k)_{k \in \mathbb{N}}$ sempre decresce o valor da função objetivo e converge em $x$ para 0 . Como a variável $y$ não contribui para o objetivo, o símbolo $(0, \infty)$ representa, em algum sentido, uma solução deste problema.

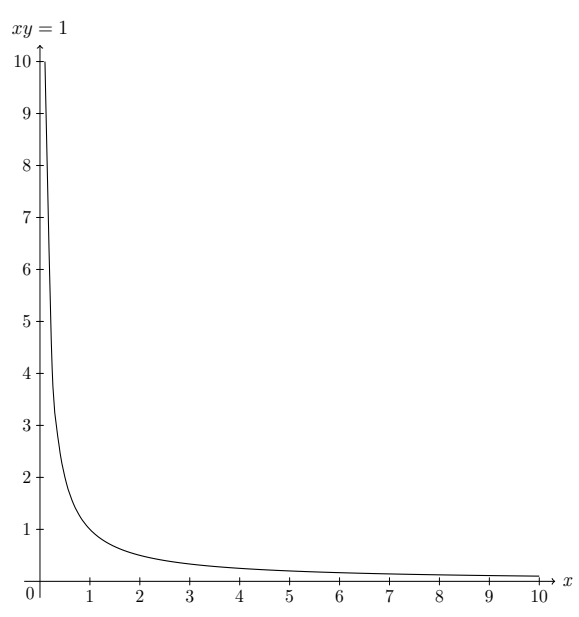

Este problema possui estratégias óbvias para restauração exata. Mas, a fim de replicar os problemas computacionais do caso original, o algoritmo foi mantido em todos os aspectos, apenas trocando os SDPs por LPs e, como este caso é bem visualizável, o resultado foi o seguinte (os pontos $\times$ são os não restaurados):

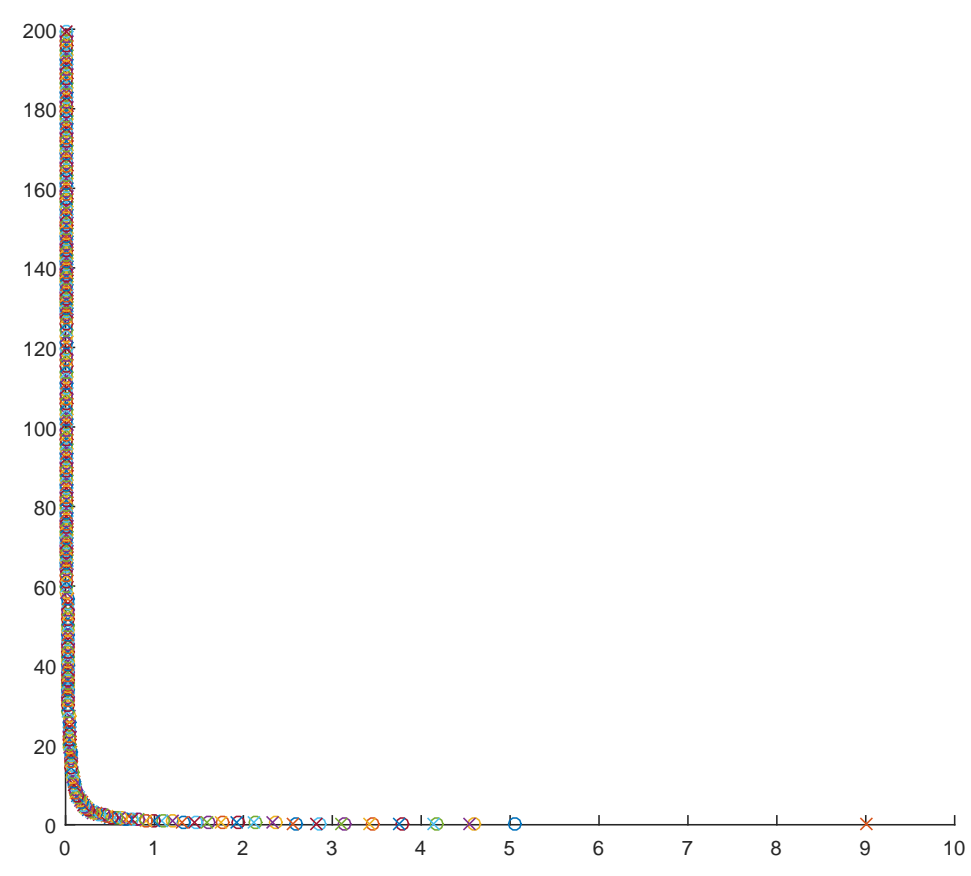

Figura 5.18: Iterandos de RI para o exemplo 12. 
Foram necessárias 183 iterações, resultando no ponto $\left(x^{\star}, y^{\star}\right)=(0.00255,392.119)$, tal que $x^{\star} y^{\star}-1=5.9 \times 10^{-13}$ e tamanho de passo $9.96 \times 10^{-6}$. Isto é, mesmo que o ponto atual ainda esteja " $10^{-3}$-longe" da solução, o passo é " $10^{-6}$-pequeno"; Logo, mesmo que haja convergência, ela será extremamente lenta a partir de certo ponto, o que é exatamente o mesmo que ocorre com o problema de cobertura por bolas abertas. Aqui, tomamos $\gamma=0.9$ e esse gráfico foi feito truncando a sequência em $y_{k} \leqslant 200$ para uma melhor visualização.

A direção a partir de um ponto $z_{k}=\left(x_{k}, y_{k}\right)$ é

$$
d_{k}=\operatorname{argmin}_{d}\left\{x_{k}+d_{x} \mid y_{k} d_{x}+x_{k} d_{y}=0, x_{k}+d_{x} \geqslant 0\right\},
$$

ou seja, $d_{k}=\left(-x_{k}, y_{k}\right)$. Sendo assim, vamos analisar a perda de viabilidade nesta direção:

$$
\begin{gathered}
\left|\left(x_{k}+\alpha d_{x}^{k}\right)\left(y_{k}+\alpha d_{y}^{k}\right)-1\right|=\left|\left(x_{k}-\alpha x_{k}\right)\left(y_{k}+\alpha y_{k}\right)-1\right|= \\
=\left|(1-\alpha)(1+\alpha) x_{k} y_{k}-1\right|=|(1-\alpha)(1+\alpha)-1|=\alpha^{2} .
\end{gathered}
$$

Da mesma forma, o gráfico de $\alpha \mapsto\left\|H\left(x_{k}+\alpha d_{k}\right)\right\|_{2}$ para o problema de cobertura também denuncia perda quadrática de viabilidade na direção obtida em (5.9), para constar:

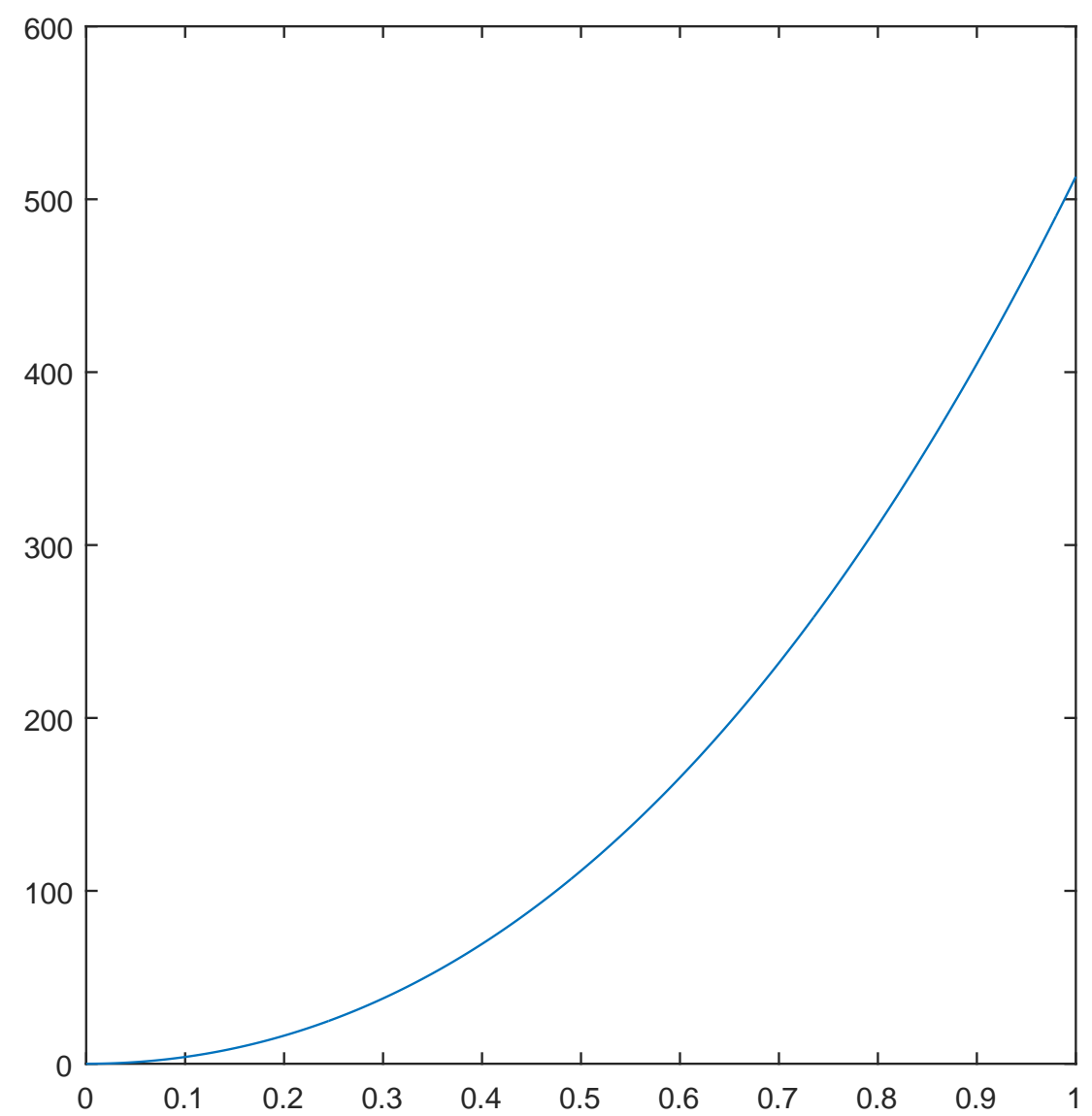

Figura 5.19: Gráfico de $\alpha \mapsto\left\|H\left(x_{k}+\alpha d_{k}\right)\right\|_{2}$ para o problema de cobertura.

As semelhanças não param por aqui, pois uma forma de melhorar o exemplo é mudando a região viável para $R_{d}:=\left\{x y^{d}=1, x \geqslant 0\right\}$, onde $d$ funciona de modo análogo ao grau dos coeficientes SOS. Eis um exemplo $\operatorname{com} d=4$ : 


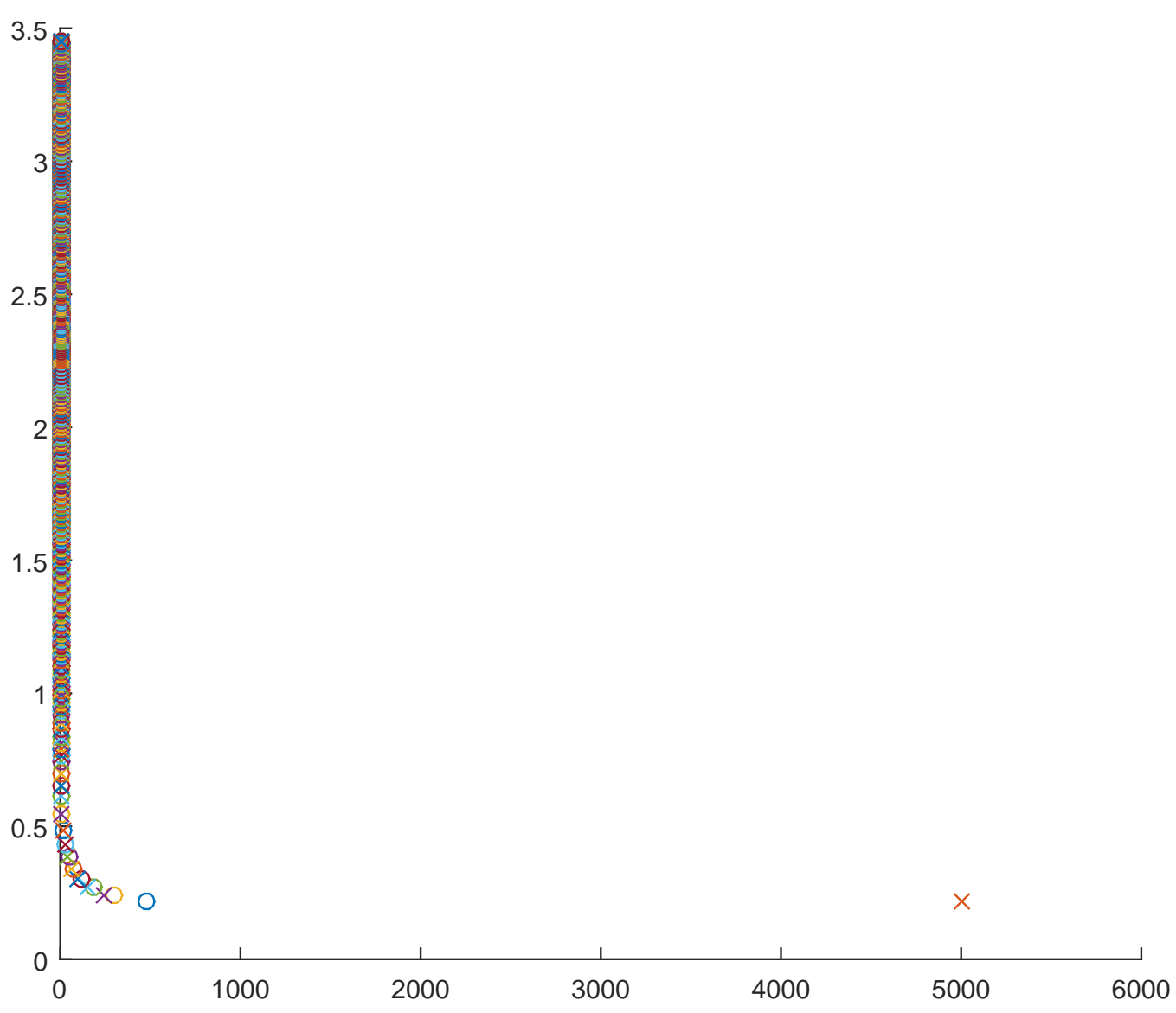

Figura 5.20: Iterandos de RI para o exemplo 12 e $d=4$.

Neste caso, mesmo com parâmetros favoráveis, o passo atinge um tamanho da ordem de $10^{-6}$ após 2075 iterações, no ponto $(x, y)=(0.0071,3.4508)$ e, portanto, temos passos " $10^{-6}$-pequenos" mesmo estando " $10^{-3}$-longe" da solução $x^{\star}=0$ e o número de iterações cresce assombrosamente ou seja, mesmo que em teoria não haja problema algum com este exemplo, na prática existem muitos. Em suma, assim como acontece com a restauração inexata aplicada ao positivstellensatz, o método converge para um ponto não-ótimo de maneira lenta e os testes comparativos indicam que a velocidade de crescimento é mais influente que o valor absoluto da variável $y$. Talvez o mesmo ocorra com $\|S\|_{2}$. Aqui, utilizamos $\gamma=0.9$.

Essa classe de problemas, com "soluções inviáveis" e variáveis passivas, aparenta ser um bom tópico para estudos mais aprofundados, uma vez que atualmente existem ferramentas que nos possibilitam considerar tais "soluções" como válidas em algum sentido, isto é, existem condições sequenciais de otimalidade.

\subsection{Testes computacionais (Stengle $+\mathrm{RI}$ )}

Se por um lado a cobertura por bolas abertas demonstra uma grande dificuldade de convergir próximo à solução, a primeira coisa a percebermos, na cobertura de Stengle, é a necessidade de muito tempo para criar o modelo simbólico do P-satz. Além disso, não é possível calcular exatamente a matriz jacobiana do problema. Logo, uma aproximação é feita toda iteração. Um dos maiores incômodos agora é a ineficácia da direção obtida em (5.9) quando o ponto atual do algoritmo é "insuficientemente viável". Em particular, 
cobrindo $B(0,1)$ com outros círculos, a direção nos centros em tais pontos é quase zero; isso foi concluído empiricamente. Não foi provado o motivo pelo qual esse comportamento está presente, mas isso faz com que seja necessário prezar pela viabilidade a fim de garantir a convergência, limitando o tamanho do passo. Isso somado à presença de muitas outras variáveis faz com que a convergência do algoritmo de restauração inexata para este caso seja extremamente lenta, não só próximo à solução, mas em todo o tempo.

O primeiro teste foi feito com um círculo cobrindo $V=B(0,1)$, iniciando com centro em $c=(0.8147,0.9058)$ e raio $r=2.21897$ e, após 50 iterações e $532.9 \mathrm{~s}$, obtivemos $c^{\star}=(0.216,0.205) \times 10^{-4}$ e $r^{\star}=0.999935$ (erro de $\left.6.5 \times 10^{-5}\right)$, com viabilidade $\left\|H^{\star}\right\|_{2}=$ $1.83 \times 10^{-8}$ e $\gamma=0.0001$. O tamanho do passo nos centros e no raio terminou na ordem de $10^{-6}$. Aqui, $d=6$ e $b=1$.

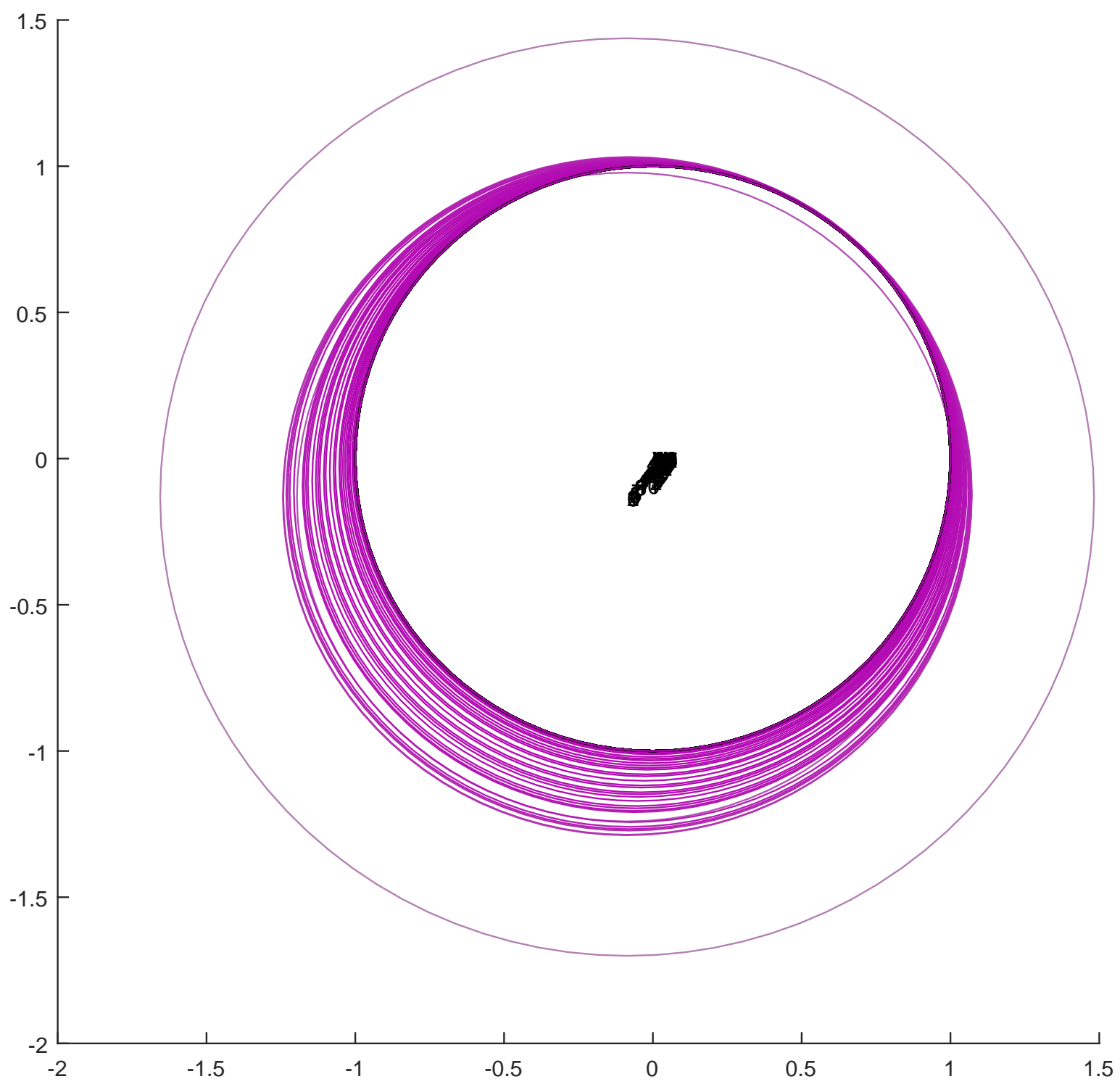

Figura 5.21: Iterandos do primeiro teste de Stengle. 
O segundo teste levou 348.4s para criar o modelo simbólico (o que é muito tempo) e se destacou por ser incrivelmente difícil para o algoritmo lidar, diferentemente do que ocorreu com o P-satz de Putinar. Após 2802 iterações e 430717.6s, obtivemos as aproximações $c_{1}^{\star}=(0.00288,-0.07088), c_{2}^{\star}=(-0.00288,0.07081)$ e $r^{\star}=1.00936$ (erro absoluto de $0.00936)$, com viabilidade $\left\|H^{\star}\right\|_{2}=3.26 \times 10^{-9}$ e parâmetro $\gamma=1$. O tamanho do passo era da ordem de $10^{-6}$ no raio e $10^{-4}$ no centro. Neste caso, $d=4$ e $2 b=2$. O ideal seria termos $d=6$, mas o algoritmo tornava-se excessivamente caro.

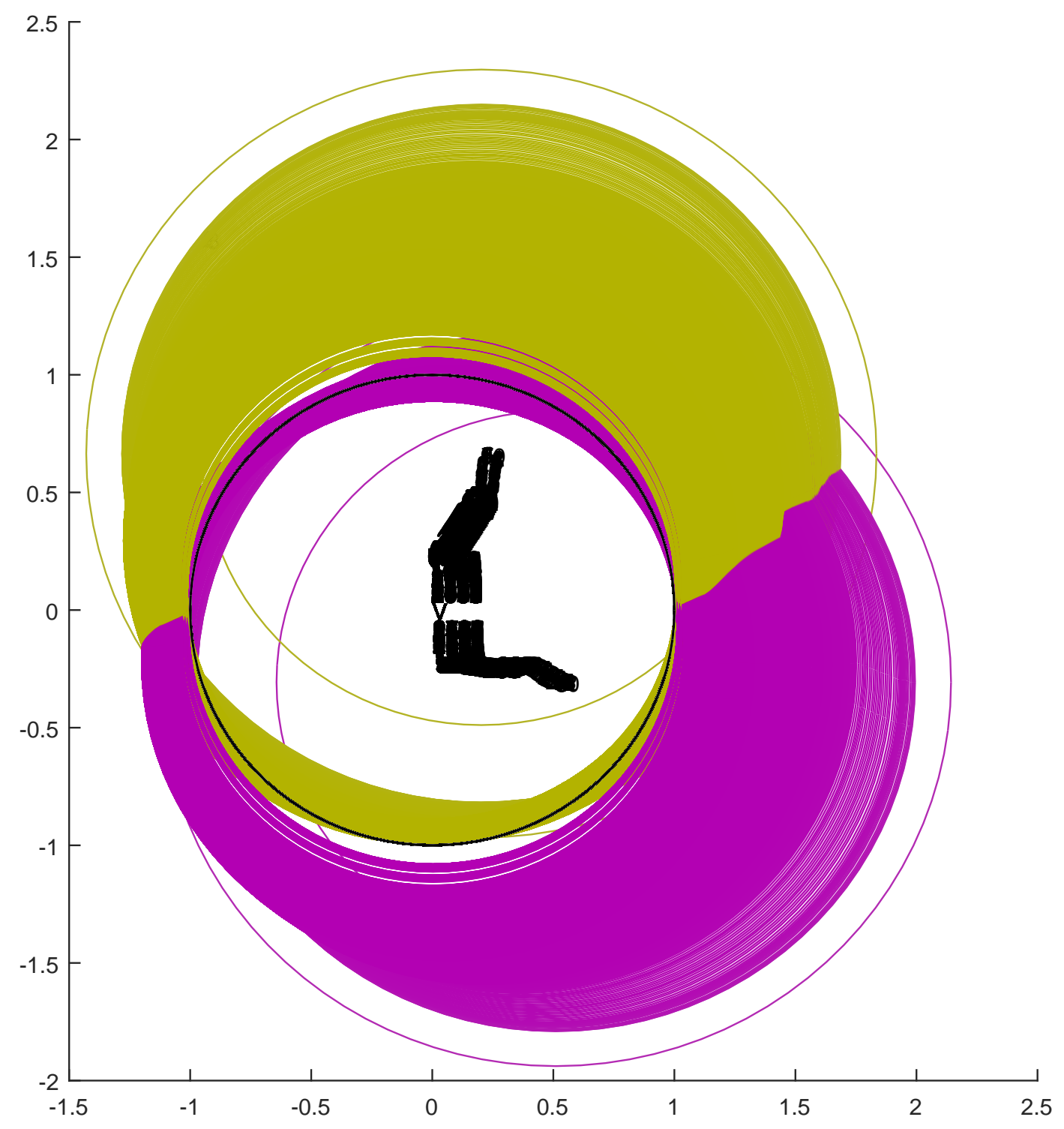

Figura 5.22: Iterandos do segundo teste de Stengle. 
A seguir, exibimos o resultado final do algoritmo para o segundo teste. É notável que o erro é inaceitável, mas suspeitamos que isso se deve ao fato do grau $d$ ser menor que o desejado.

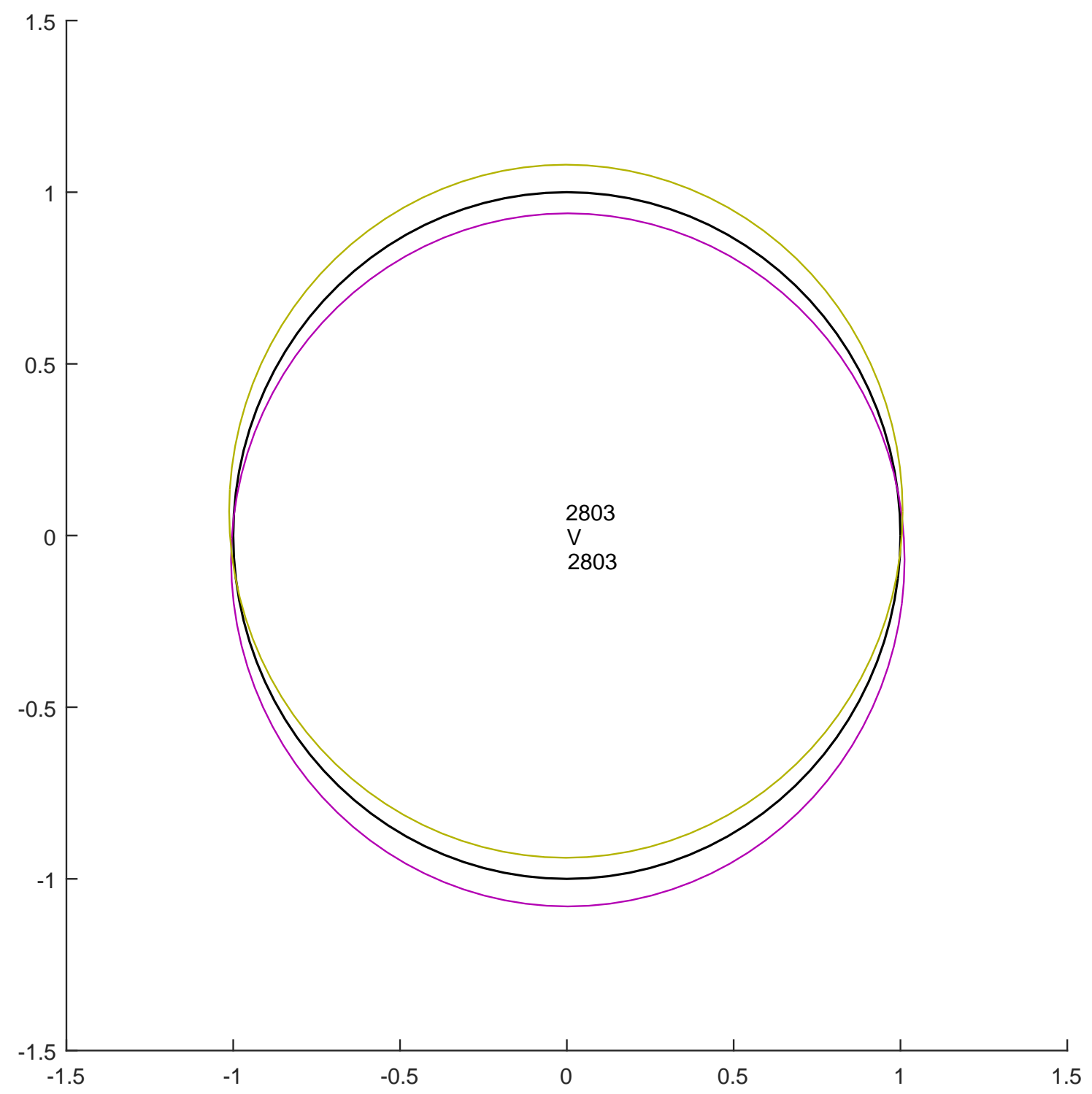

Figura 5.23: Solução do segundo teste de Stengle.

O terceiro teste, com três círculos, $d=6$ e $2 b=2$, levou 17790.3 s para criar o modelo simbólico, 540.3s para calcular o raio inicial e apenas 12 iterações do método custaram pelo menos 12 horas. Isso indica inviabilidade computacional. Portanto, esse teste foi cancelado, mas ainda o mencionamos para ilustrar o aumento da complexidade em função dos dados de entrada ao utilizar o P-satz de Stengle.

\subsection{Considerações empíricas, heurísticas e sugestões}

Reunimos nesta seção algumas observações levantadas durante os testes computacionais, sem profundidade suficiente para serem demonstradas, mas presentes o suficiente para serem dignas de menção. 


\subsubsection{Sobre as variáveis inteiras}

Após realizar diversos testes, pudemos notar algumas características interessantes sobre as variáveis inteiras $d$ e $b$ presentes no problema.

Pelo Teorema 24, existe uma limitação para $d$ no $P$-satz de Putinar ao verificar se um polinômio é positivo num conjunto não vazio. Contudo, o fazemos em conjuntos vazios, de modo que este resultado não é diretamente aplicável. Suspeitamos que, apesar de não provada, a conclusão é válida. Mesmo assim, em geral, este limitante é muito grande para ser implementado. Por outro lado, na prática, foi constatado que um $d$ menor que o recomendado não implica em falha total do algoritmo de cobertura, mas apenas limita sua precisão (isto é, quando maior for $d$, mais próximo da solução será possível chegar). É possível comparar isso com a qualidade da discretização presente nas abordagens clássicas, uma vez que, para melhorar a solução, é necessário reformular o problema com um grau maior, o que é um processo caro.

Tratando agora do $P$-satz de Stengle, a variável $b$ parece estar relacionada com $d$, de modo que, quando maior for $b$, maior deve ser $d$. Para esta não há limitante teórico.

\subsubsection{Heurísticas}

Algumas ideias surgiram durante a execução deste projeto, mas não possuem tanto respaldo teórico quanto a restauração inexata. Foram decisões tomadas com base em observações ou comportamentos em casos isolados. Estamos abusando do termo "heurística" aqui, porque tais decisões não deixam o algoritmo necessariamente mais rápido, mas apenas mais simples.

A primeira heurística é um algoritmo baseado no esquema de "pontos interiores", no qual começamos com uma cobertura viável, calculamos o passo de (5.9) (que está bem definido a partir de um ponto viável) e fazemos busca linear resolvendo o SDP (5.8) a cada tentativa de parâmetro $\alpha$.

Algoritmo 5 (Pontos interiores). Receba como entrada uma cobertura viável $\left(r_{0}, C^{0}, S^{0}\right)$. Para $k \in \mathbb{N}$ :

1. Calcule um passo d $d^{k}$ solução de 5.9;

2. Defina $\alpha_{k} \leftarrow 1$;

3. Se existe $S_{k}$ tal que $H\left(r_{k}+\alpha_{k} d_{r}^{k}, C^{k}+\alpha_{k} d_{C}^{k}, S_{k}\right)=0$, volte ao Passo 2;

4. Señ̃o, faça $\alpha_{k} \leftarrow \alpha_{k} / 2$ e retorne ao Passo 3.

5. Pare quando um critério for satisfeito.

Este algoritmo funciona particularmente bem com o P-satz de Stengle, uma vez que a viabilidade estrita implica numa ótima aproximação numérica do jacobiano. Alguns testes foram feitos com este algoritmo e uma das características mais notórias é o pequeno número de iterações até a convergência, para os mesmos resultados, mesmo que cada uma delas seja muito mais custosa que as do Algoritmo 4. Por exemplo, para cobrir $V=B(\overrightarrow{0}, 1)$ com três círculos, obtivemos o raio ótimo $r^{\star}=0.866545$ (erro absoluto de 0.00052 ) em apenas 32 iterações, parando com tamanho de passo na ordem de $10^{-6}$.

A segunda heurística é baseada no gráfico da perda de viabilidade (Figura 5.19) apresentado no Exemplo 12. Assumimos que este comportamento se repete no caso geral, o 
que não parece ser uma hipótese muito absurda. Como o ganho de otimalidade é garantido sempre que $d_{r}^{k}<0$, então a função de mérito no Algoritmo 4 controla apenas a perda de viabilidade, sendo assim, conhecendo o comportamento da viabilidade ao longo da direção $d_{k}$, é possível propor uma função de mérito mais simples, isto é, quando

$$
\left\|H\left(r_{k}, C^{k}, S^{k}\right)\right\|_{2}-\left\|H\left(\left(r_{k}, C^{k}, S^{k}\right)+\alpha_{k} d^{k}\right)\right\|_{2}<\sigma_{k},
$$

o tamanho de passo $\alpha_{k}$ pode ser aceito. Neste caso, quanto menor $\sigma_{k}$, maior a preferência pela viabilidade. Os resultados com este algoritmo, em geral, são semelhantes aos do Algoritmo 4.

\subsubsection{Sugestões para trabalhos futuros}

Compilaremos aqui algumas das sugestões que recebemos e ideias não implementadas; é claro, não se trata de negligência, mas de pequenos impeditivos para o pleno aproveitamento dessas ideias, presentes até o momento.

- A técnica de restauração baseada em incrementar o raio até (5.8) ser satisfeita, ou seja, caminhando na direção $(1,0,0, \ldots, 0)$, pode ser substituída por uma instância de (5.9) com as matrizes $S^{k}$ fixadas.

- Pode-se substituir o paradigma de restauração inexata por um método baseado no Gradiente Espectral Projetado ( $S P G$ ), uma vez que a projeção em $\mathcal{S}^{n}$ é uma tarefa relativamente simples.

- É possível explorar as condições KKT para SDPs não lineares a fim de obter um algoritmo mais significativo, como foi proposto em [BHM14].

- As restrições semidefinidas $S_{i} \succeq 0$ podem ser descritas como restrições de desigualdade na forma $\lambda_{\min }\left(S_{i}\right) \geqslant 0$, o que nos permite enxergar (5.5) e (5.3) como problemas de otimização não linear e, sob certas hipóteses, eventuais conclusões acerca de "soluções inviáveis" (como no Exemplo 12) podem ser empregadas.

- Em casos pequenos, nada impede o uso complementar de ambas as versões do positivstellensatz, obtendo uma boa aproximação do ótimo com o P-satz de Putinar e, em seguida, refinando esse resultado com o de Stengle.

- As esferas podem ser substituídas por quaisquer outras regiões limitadas por curvas de nível de uma função polinomial sem muita dificuldade teórica, por exemplo, ou outras normas. 


\section{Capítulo 6}

\section{Conclusão}

Tratando-se de algo sabidamente difícil, tanto no sentido teórico quanto computacional, o problema de cobertura vem sendo abordado de maneiras engenhosas nos últimos tempos. Mas, em geral, o mesmo modelo aproximado discretizado que simplifica enormemente o problema original também carrega características indesejáveis, dependendo da situação. A fim de atender a esses casos, apresentamos uma nova abordagem sem discretizações, que portanto não perde informações do conjunto original. Para isso, exploramos o positivstellensatz de Stengle e uma versão alternativa de Putinar.

Devido à íntima relação entre somas de quadrados e matrizes semidefinidas positivas, o problema de cobertura pode ser reduzido a um SDP não linear. Embora existam métodos para resolução deste tipo de problema, optamos por aproveitar suas particularidades e resolvê-lo com o paradigma de restauração inexata, já que existe uma técnica trivial de restauração e os subproblemas associados ao paradigma são todos SDPs lineares.

A cobertura por bolas fechadas está associada ao positivstellensatz de Stengle e a com bolas abertas, ao de Putinar. A primeira é robusta, tem muitas variáveis matriciais e uma variável inteira, além do grau dos coeficientes. Isso somado ao fato da viabilidade exigir preferência a aplicar a restauração inexata, torna a convergência do método demasiadamente lenta. Já a segunda é bem mais simples, pois o número de variáveis matriciais cresce linearmente com os dados de entrada. Mas todas essas vantagens carregam a infeliz propriedade dos coeficientes tenderem ao infinito conforme a sequência gerada pelo algoritmo converge ao ponto ótimo, o que acarreta em grandes dificuldades computacionais. Ainda nesse âmbito, vale comentar que, enquanto $r^{\star}+\varepsilon$ pode ser considerado inviável para Putinar, $r^{\star}-\varepsilon$ pode ser considerado viável para Stengle, isto é, não é possível fugir das imprecisões computacionais. Contudo, foram obtidas boas aproximações para as coberturas que possuem soluções conhecidas e matematicamente provadas. Além disso, nossa abordagem tem um forte respaldo teórico e um bom grau de inovação e abrangência.

Em geral, acreditamos que a técnica apresentada neste trabalho possa servir como uma alternativa válida aos métodos usuais, sujeito às peculiaridades da aplicação, dado o devido refinamento computacional. A versão do positivstellensatz de Putinar aparenta ser a mais adequada que a de Stengle, na maioria dos casos, uma vez que escala bem com os dados de entrada e, quanto ao grau dos coeficientes SOS, estes aparentam ser mais dependentes do grau dos polinômios que descrevem $V$, em comparação com o número de esferas. 



\section{Bibliografia}

[AC68] J. Abadie e J. Carpentier. "Generalization of the Wolfe reduced-gradient method to the case of nonlinear constraints". Em: Optimization (1968), pp. 37-47.

[Ahm17] A. A. Ahmadi. "Sums of Squares (SOS) Techniques: An Introduction". Em: Notas de aula do curso ORF523 (2017). URL: http://www.princeton.edu/ amirali/Public/Teaching/ORF523/S16/ORF523_S16_Lec15.pdf.

[BBM15] E. G. Birgin, L. F. Bueno e J. M. Martínez. "Assessing the Realiability of General Purpouse Inexact Restoration Methods". Em: Journal of Computational and Applied Mathematics (2015), pp. 1-16. ISSN: 0377-0427. DOI: 10.1016/j. cam.2014.12.031. URL: http://www.sciencedirect.com/science/article/pii/ S0377042714005871.

[BHM14] L. F. Bueno, G. Haeser e J. M. Martínez. "A Flexible Inexact-Restoration Method for Constrained Optimization". Em: Journal of Optimization Theory and Applications (2014), pp. 188-208. ISSN: 0022-3239. DOI: 10.1007/s10957014-0572-0. URL: https://doi.org/10.1007/s10957-014-0572-0.

[BHM15] L. F. Bueno, G. Haeser e J. M. Martínez. “An Inexact Restoration Approach to Optimization Problems with Multiobjective Constraints under Weighted-Sum Scalarization". Em: Optimization Letters (2015), pp. 1315-1325. ISSN: 18624472. DOI: 10.1007/s11590-015-0928-x. URL: https://doi.org/10.1007/s11590015-0928-x.

[BKM17] E. G. Birgin, N. Krejic e J. M. Martínez. "On the Employment of Inexact Restoration for the Minimization of Functions Whose Evaluation is Subject to Errors". Em: Mathematics of Computation (2017). DOI: 10.1090/mcom/3246. URL: https://www.ime.usp.br/ egbirgin/publications/bkm2015b.pdf.

[BKM18] E. G. Birgin, N. Krejic e J. M. Martínez. "Iteration and Evaluation Complexity on the Minimization of Functions Whose Computation is Intrinsically Inexact". Em: (2018). URL: https://www.ime.usp.br/ egbirgin/publications/bkm2016. pdf.

[Ble06] G. Blekherman. "There are significantly more nonegative polynomials than sums of squares". Em: Israel Journal of Mathematics (2006), pp. 153-355. DOI: 10.1007/BF02771790. URL: https://doi.org/10.1007/BF02771790.

[Cou09] S. C. Coutinho. Polinômios e Computação Algébrica. Rio de Janeiro, RJ: IMPA, 2009.

[FF10] A. Fischer e A. Friedlander. "A New Line Search Inexact Restoration Approach for Nonlinear Programming". Em: A. Comput Optim Appl (2010), p. 333. DOI: 10.1007/s10589-009-9267-0. URL: https://doi.org/10.1007/s10589-009-9267-0. 
[FR16] L. Fejér e F. Riesz. "Über trigonometrische Polynome". Em: J. Reine Angew. Math. (1916), pp. 53-82. DOI: 10.1515/crll.1916.146.53. URL: https://doi.org/ 10.1515/crll.1916.146.53.

[Fri18] E. Friedman. "Circles Covering Circles". Em: Página da Stetson University (2018). URL: http://www2.stetson.edu/〜efriedma/circovcir/.

[Gha08] L. E. Ghaoui. "SDP Duality". Em: Notas de aula do curso EE227A: Convex Optimization and Applications (2008). URL: https://people.eecs.berkeley.edu/ 〜 wainwrig/ee227a/SDP_Duality.pdf.

[Hil93] D. Hilbert. "Über ternäre definite Formen”. Em: Acta Math. 17 (1893), pp. 169197. DOI: 10.1007/BF02391990. URL: https://doi.org/10.1007/BF02391990.

[Las10] J. B. Lasserre. Moments, Positive Polynomials and Their Applications. Covent Garden, London: Imperial College Press, 2010, pp. 15-38. DOI: 10.1007/ s10208-011-9092-6. URL: https://doi.org/10.1007/s10208-011-9092-6.

[Lau09] M. Laurent. "Sums of Squares, Moment Matrices and Optimization Over Polynomials". Em: Emerging Applications of Algebraic Geometry. Ed. por M. Putinar e S. Sullivant. New York, NY: Springer New York, 2009, pp. 11-49. ISBN: 978-0-387-09686-5. DOI: 10.1007/978-0-387-09686-5_7. URL: https: //doi.org/10.1007/978-0-387-09686-5_7.

[Lö09] J. Löfberg. "Pre- and post-processing sum-of-squares programs in practice". Em: IEEE Transactions on Automatic Control 54.5 (2009), pp. 1007-1011. DOI: 10.1109/CACSD.2004.1393890. URL: https://doi.org/10.1109/CACSD. 2004.1393890 .

[MP00] J. M. Martínez e E. A. Pilotta. "Inexact Restoration Algorithm for Constrained Optimization". Em: Journal of Optimization Theory and Applications (2000), pp. 135-163. DOI: 10.1023/A:1004632923654. URL: https://www.ime.unicamp. $\mathrm{br} /{ }^{\sim}$ martinez/mp.pdf.

[MP05] J. M. Martínez e E. A. Pilotta. "Inexact Restoration Methods for Nonlinear Programming: Avances and Perspectives". Em: Optimization and Control with Applications (2005). DOI: 10.1007/0-387-24255-4_12. URL: https://doi.org/ 10.1007/0-387-24255-4_12.

[Man04] M. Manetti. "Nullstellensatz per Tutti". Em: Pubbl. Cent. Ric. Mat. Ennio Giorgi (2004). URL: http://www1.mat.uniroma1.it/people/manetti/dispense/ nullstellen.pdf.

[Mot67] T. S. Motzkin. "The Arithmetic-Geometric Inequality". Em: Inequalities (1967), pp. 205-224.

[NS08] J. Nie e M. Schweighofer. "On the complexity of Putinar's Positivstellensatz". Em: Journal of Complexity 23.1 (2008), pp. 135-150. DOI: 10.1016/j.jco.2006. 07.002. URL: https://doi.org/10.1016/j.jco.2006.07.002.

[PBT13] P. A. Parrilo, G. Blekherman e R. R. Thomas. Semidefinite Optimization and Convex Algebraic Geometry. MOS-SIAM Series on Optimization, 2013, pp. 3202. ISBN: 978-1-61197-228-3. DOI: $10.1137 / 1.9781611972290$. URL: https: //doi.org/10.1137/1.9781611972290.

[PR00] V. Powers e B. Reznick. "Polynomials That are Positive on an Interval". Em: Transactions of the American Mathematical Society (2000). DOI: 10.2307/ 221758. URL: https://faculty.math.illinois.edu/ reznick/pos.pdf. 
[PS98] G. Polyá e G. Szegö. Problems and Theorems in Analysis II. Berlin, Heldelberg: Springer-Verlag, 1998. DOI: 10.1007/978-3-642-61905-2. URL: https://doi. org/10.1007/978-3-642-61905-2.

[Pru86] J. Prussing. "The Principal Minor Test for Positive Semidefinite Matrices". Em: Journal of Guidance Control and Dynamics (1986), pp. 121-122. DOI: 10.2514/3.20077. URL: prussing.ae.illinois.edu/semidef.pdf.

[Put93] M. Putinar. "Positive Polynomials on Compact Semi-algebraic Sets". Em: Indiana University Mathematics Journal 42 (1993), pp. 969-984. URL: http: //www.jstor.org/stable/24897130.

[Ros60] J. B. Rosen. "The gradient projection method for nonlinear programming, Part 1, linear constraints". Em: SIAM Journal on Applied Mathematics 8 (1960), pp. 181-217.

[Ros61] J. B. Rosen. "The gradient projection method for nonlinear programming, Part 2, nonlinear constraints". Em: SIAM Journal on Applied Mathematics 9 (1961), pp. 514-532.

[Sch91] K. Schmüdgen. "The K-moment problem for compact semi-algebraic sets". Em: Mathematische Annalen (1991), pp. 203-289. DOI: 10.1007/BF01446568. URL: https://doi.org/10.1007/BF01446568.

[Ste74] G. Stengle. "A nullstellensatz and a positivstellensatz in semialgebraic geometry". Em: Mathematische Annalen (1974). DOI: 10.1007/BF01362149. URL: https://doi.org/10.1007/BF01362149.

[Stu99] J. F. Sturm. "Using SeDuMi 1.02, A Matlab Toolbox for Optimization Over Symmetric Cones". Em: Optimization Methods and Software (1999), pp. 625653. DOI: $10.1080 / 10556789908805766$. URL: https:// doi .org/10.1080/ 10556789908805766 .

[TTT99] K.-C. Toh, M. J. Todd e R. H. Tutuncu. "SDPT3 - a Matlab software package for semidefinite programming". Em: Optimization Methods and Software (1999), pp. 545-581. DOI: 10.1080/10556789908805762. URL: https://doi.org/ $10.1080 / 10556789908805762$.

[Tan15] V. Y. F. Tan. "Simplified Proof of Slater's Theorem for Strong Duality". Em: Notas de aula do curso EE5138R (2015). URL: https://www.ece.nus.edu.sg/ stfpage/vtan/ee5138/slater.pdf.

[VLX15] H. Venceslau, D. Lubke e A. Xavier. "Optimal covering of solid bodies by spheres via the hyperbolic smoothing technique". Em: Optimization Methods and Software (2015). DOI: 10.1080/10556788.2014.934686. 



\section{Lista de Figuras}

2.1 Exemplo de cobertura com o menor raio possível, mas não a menor área. 18

2.2 Um exemplo da desvantagem na discretização interior. . . . . . . . . . . 19

2.3 Um exemplo da desvantagem na discretização exterior. . . . . . . . . . . 19

3.1 Um exemplo de conjunto semialgébrico, em que cada uma das regiões coloridas (e as linhas) são conjuntos semialgébricos básicos. . . . . . . . . . 28

3.2 Representação de $\mathcal{P}_{n}$ e $\Sigma_{n} \ldots \ldots \ldots \ldots \ldots \ldots \ldots$. . . . . . . . . . . . . . . . .

4.1 Representação de um espectraedro, como interseção de $\mathcal{S}_{+}^{n}$ com um subespaço afim. . . . . . . . . . . . . . . . 46

5.1 Os pontos estrelados satisfazem a descrição do Lema 5. . . . . . . . . . . 64

5.2 O único ponto a satisfazer a descrição do Lema 5 está no interior de $V$. . . 64

5.3 Iterandos do primeiro teste cobrindo um círculo. . . . . . . . . . . . 70

5.4 Iterandos do segundo teste cobrindo um círculo. . . . . . . . . . . . 71

5.5 Solução do segundo teste cobrindo um círculo. . . . . . . . . . . . . 72

5.6 Iterandos do terceiro teste cobrindo um círculo. . . . . . . . . . . . . 73

5.7 Solução do terceiro teste cobrindo um círculo. . . . . . . . . . . . . . 74

5.8 Iterandos do quarto teste cobrindo um círculo. . . . . . . . . . . . . 75

5.9 Solução do quarto teste cobrindo um círculo. . . . . . . . . . . . 76

5.10 Solução de K. Bezdek para seis círculos. . . . . . . . . . . . 76

5.11 Iterandos do primeiro teste cobrindo um coração. . . . . . . . . . . . . 77

5.12 Solução do primeiro teste cobrindo um coração. . . . . . . . . . . . . . . 77

5.13 Iterandos do segundo teste cobrindo um coração. . . . . . . . . . . . . . . 78

5.14 Solução do segundo teste cobrindo um coração. . . . . . . . . . . . . 79

5.15 Solução do terceiro teste cobrindo um coração. . . . . . . . . . . . . 80

5.16 Iteração inicial e final do terceiro teste cobrindo um coração. . . . . . . . . 81

5.17 Gráfico de $r \mapsto\|S(r)\|_{2}$ no problema de cobertura com $V=B(\overrightarrow{0}, 1)$ e $m=1.82$

5.18 Iterandos de RI para o exemplo $12 \ldots \ldots \ldots$. . . . . . . . 83

5.19 Gráfico de $\alpha \mapsto\left\|H\left(x_{k}+\alpha d_{k}\right)\right\|_{2}$ para o problema de cobertura. . . . . . 84

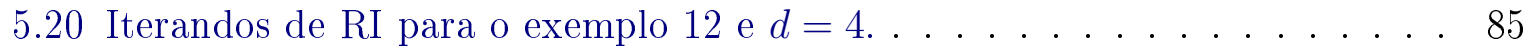

5.21 Iterandos do primeiro teste de Stengle. . . . . . . . . . . . . 86

5.22 Iterandos do segundo teste de Stengle. . . . . . . . . . . . . . . . . . . 87

5.23 Solução do segundo teste de Stengle. . . . . . . . . . . . . . 88 